\title{
PERIODIC ORBITS OF MAPS OF $Y$
}

\author{
LLUIS ALSEDȦ, JAUME LLIBRE AND MICHAX MISIUREWICZ
}

\begin{abstract}
We introduce some notions that are useful for studying the behavior of periodic orbits of maps of one-dimensional spaces. We use them to characterize the set of periods of periodic orbits for continuous maps of $Y=\left\{z \in \mathbf{C}: z^{3} \in[0,1]\right\}$ into itself having zero as a fixed point. We also obtain new proofs of some known results for maps of an interval into itself.
\end{abstract}

\section{INTRODUCTION}

In recent years, there has been growing interest in studying the periodic orbits of maps of one-dimensional spaces. One of the first results, and the most spectacular one, is the Šarkovskii theorem. We shall recall it to the reader.

Let $I$ be the family of all continuous maps of the interval $[0,1]$ into itself (one can take any closed interval, but we choose $[0,1]$ to fix notation). For $f \in \mathbf{I}$, if $f^{n}(x)=x$, then we call the set $\left\{x, f(x), \ldots, f^{n-1}(x)\right\}$ a periodic orbit of $f$, and its period is the smallest positive integer $m$ such that $f^{m}(x)=$ $x$. We denote by $\operatorname{Per}(f)$ the set of periods of all periodic orbits of $f$.

Let $\mathbf{N}$ be the set of all positive integers. The Sarkovskil ordering of $\mathbf{N}$ is

$$
\begin{array}{r}
3,5,7,9, \ldots, 2.3,2.5,2.7,2.9, \ldots, \\
2^{2} .3,2^{2} .5,2^{2} .7,2^{2} .9, \ldots, \ldots, 2^{3}, 2^{2}, 2,1 .
\end{array}
$$

If $k$ stands to the right of $n$ in the above ordering, we shall write $k>_{s} n$. If $k=2^{p} \cdot k^{\prime}$ and $n=2^{q} \cdot n^{\prime}$, where $k^{\prime}$ and $n^{\prime}$ are odd, then we have $k>_{s} n$ if and only if one of the following cases occurs:

(i) $k^{\prime}>1, n^{\prime}>1, p>q$.

(ii) $k^{\prime}>1, n^{\prime}>1, p=q, k^{\prime}>n^{\prime}$.

(iii) $k^{\prime}=1, n^{\prime}>1$.

(iv) $k^{\prime}=1, n^{\prime}=1, p<q$.

We denote $S(n)=\{n\} \cup\left\{k: k>_{s} n\right\}$ for $n \in \mathbf{N}, S\left(2^{\infty}\right)=\left\{2^{i}: i=\right.$ $0,1, \ldots\}$, and $\mathbf{N}_{s}=\mathbf{N} \cup\left\{2^{\infty}\right\}$.

Received by the editors May 15, 1987.

1980 Mathematics Subject Classification (1985 Revision). Primary 54H20.

Key words and phrases. Periodic orbit, primary orbit, set of periods, Šarkovskii theorem.

This paper was made possible by an invitation of the Centre de Recerca Matematica of the Institut d'Estudis Catalans to M. Misiurewicz. The first two authors have been partially supported by a grant of CAICYT no. 3534 183.C3. 
Šarkovskii Theorem. (a) If $f \in \mathbf{I}$, then $\operatorname{Per}(f)=S(n)$ for some $n \in \mathbf{N}_{s}$.

(b) If $n \in \mathbf{N}_{s}$, then there exists $f \in \mathbf{I}$ such that $\operatorname{Per}(f)=S(n)$.

Further research starting at this point can go in at least six directions:

(1) Replace an interval and I by another space and another class of maps.

(2) Replace periodic orbits by more general orbits.

(3) Investigate closer the behavior of periodic orbits.

(4) Study other orbits in the presence of periodic ones.

(5) Derive some information about topological entropy.

(6) Try to simplify the proofs.

There have been many attempts to pursue these goals; for instance, see the references.

The aim of this paper is to develop some tools that are useful for moving in the first direction and to use them in a relatively simple case. This also requires some work in the third direction. As a reward we get better insight into some already known results, including the Šarkovskii theorem, the dependence between simple and minimal orbits (see [Št, B13, ALS]), and the results of Mumbrú [Mu].

When trying to generalize the Šarkovskii theorem, one should decide first how such a generalization should look. We believe that it should be a complete characterization of possible sets $\operatorname{Per}(f)$ for $f$ from a class of maps under consideration. The fact that in the Sarkovskii theorem there appears just a total ordering of all positive integers seems to be only a coincidence. Therefore, theorems stating that for $f$ from some class of maps the existence of periodic orbits of some periods implies the existence of periodic orbits of some other periods should be regarded as generalizations of the theorem of $\mathrm{Li}$ and Yorke [LY] rather than of the Šarkovskii theorem.

The main tool we use to generalize the Šarkovskii theorem is the notion of a primary orbit. If we consider some class $\mathbf{X}$ of maps and $P$ is a periodic orbit of a map $f \in \mathbf{X}$, then $P$ will be called primary if there exists $g \in \mathbf{X}$ such that $\left.f\right|_{P}=\left.g\right|_{P}$ and there is no other periodic orbit of $g$ of the same period as $P$ (they are the same as $\rightarrow$-minimal orbits of $[\mathrm{Ba}]$ ).

The notion of a primary orbit plays a role similar to that of the notion of a minimal orbit ([ั̌st, Bl3, C, ALS, H]; a periodic orbit $P$ of period $m$ of $f \in \mathbf{I}$ was called minimal if $\operatorname{Per}(f)=S(m))$. In fact, the notions are similar to each other (see Remark 9.2). However, the notion of a primary orbit is better since it depends only on the behavior of a map on this orbit and its definition does not use the a priori knowledge of Šarkovskii ordering (hence it is much more general).

A general scheme for using the notion of primary orbits will be:

(1) Prove the following condition:

If $f \in \mathbf{X}$ has some periodic orbit, then it has a primary orbit of the same period. 
(2) Find all primary orbits of maps $f \in \mathbf{X}$.

(3) Classify them.

(4) Find dependences between their occurrences.

Of course, this scheme works only for classes $\mathbf{X}$ for which $(*)$ is true. Otherwise, one has to look for some better tools.

We are going to apply this scheme to the family $\mathbf{Y}$ of all continuous maps $f$ of the space $Y=\left\{z \in C: z^{3} \in[0,1]\right\}$ into itself for which $f(0)=0$. To describe the result that we obtain, we need to introduce two new orderings.

Green ordering is the ordering of $\mathbf{N} \backslash\{2\}$ :

$$
\begin{aligned}
& 5,8,4,11,14,7,17,20,10,23,26,13, \ldots, \\
& 3.3,3.5,3.7, \ldots, 3.2 .3,3.25,3.2 .7, \ldots, 3.2^{2} .3,3.2^{2} .5,3.2^{2} .7, \ldots, \\
& \ldots, 3.2^{3}, 3.2^{2}, 3.2,3.1,1 .
\end{aligned}
$$

The first part of this ordering can be rewritten as

$$
6-1,6+2,3+1,26-1,26+2,2.3+1,3.6-1,3.6+2,3.3+1, \ldots
$$

If $k$ stands to the right of $n$ in the above ordering, we shall write $k>_{g} n$.

In this paper, the symbol $\equiv$ will denote congruences mod 3 .

We have $k>_{g} n$ (remember that $k \neq 2$ and $n \neq 2$ ) if and only if $n>1$ and one of the following cases occurs:

(i) $k \not \equiv 0, n \not \equiv, k \equiv n, k>n$.

(ii) $k \equiv 1, n \equiv 2,2 k \geq n$.

(iii) $k \equiv 2, n \equiv 1, k>2 n$.

(iv) $k \equiv 0, n \neq 0$.

(v) $k \equiv 0, n \equiv 0, k / 3>_{s} n / 3$.

(vi) $k=1$.

Red ordering is the ordering of $\mathbf{N} \backslash\{2,4\}$ :

$$
\begin{aligned}
& 7,10,5,13,16,8,19,22,11,25,28,14, \ldots, \\
& 3.3,3.5,3.7, \ldots, 3.2 .3,3.2 .5,3.2 .7, \ldots, 3.2^{2} .3,3.2^{2} .5,3.2^{2} .7, \ldots, \\
& \ldots, 3.2^{3}, 3.2^{2}, 3.2,3.1,1 .
\end{aligned}
$$

The first part of this ordering can be rewritten as

$$
6+1,6+4,3+2,2.6+1,2.6+4,2.3+2,3.6+1,3.6+4,3.3+2, \ldots
$$

If $k$ stands to the right of $n$ in the above ordering, we shall write $k>_{r} n$. We have $k>r_{r} n$ (remember that $k, n \notin\{2,4\}$ ) if and only if $n>1$ and one of the following cases occurs:

(1) $k \not \equiv 0, n \not \equiv 0, k \equiv n, k>n$.

(2) $k \equiv 2, n \equiv 1,2 k \geq n$.

(3) $k \equiv 1, n \equiv 2, k>2 n$.

(4) $k \equiv 0, n \not \equiv 0$.

(5) $k \equiv 0, n \equiv 0, k / 3>{ }_{s} n / 3$.

(6) $k=1$. 
We denote

$$
\begin{aligned}
& G(n)=\{n\} \cup\left\{k: k>_{g} n\right\} \quad \text { for } n \in \mathbf{N} \backslash\{2\}, \\
& R(n)=\{n\} \cup\left\{k: k>_{r} n\right\} \quad \text { for } n \in \mathbf{N} \backslash\{2,4\},
\end{aligned}
$$

and additionally

$$
G\left(3.2^{\infty}\right)=R\left(3.2^{\infty}\right)=\{1\} \cup\left\{3 . i: i \in S\left(2^{\infty}\right)\right\} .
$$

We also denote $\mathbf{N}_{g}=(\mathbf{N} \backslash\{2\}) \cup\left\{3.2^{\infty}\right\}$ and $\mathbf{N}_{r}=(\mathbf{N} \backslash\{2,4\}) \cup\left\{3.2^{\infty}\right\}$.

Main Theorem. (a) If $f \in \mathbf{Y}$, then $\operatorname{Per}(f)=S\left(n_{s}\right) \cup G\left(n_{g}\right) \cup R\left(n_{r}\right)$ for some $n_{s} \in \mathbf{N}_{s}, n_{g} \in \mathbf{N}_{g}$, and $n_{r} \in \mathbf{N}_{r}$.

(b) If $n_{s} \in \mathbf{N}_{s}, n_{g} \in \mathbf{N}_{g}$, and $n_{r} \in \mathbf{N}_{r}$ then there exists $f \in \mathbf{Y}$ for which $\operatorname{Per}(f)=S\left(n_{s}\right) \cup G\left(n_{g}\right) \cup R\left(n_{r}\right)$.

The paper is organized as follows. In $\S 1$ we give basic definitions and prove some preliminary results. In $\S 2$ we give different characterizations of primary orbits and prove that $(*)$ holds for $Y$. In $\S 3$ we introduce the notion of extensions, which allow us to construct new periodic orbits from given ones, and we prove some properties of extensions. In $\S 4$ we define the periodic orbits which are candidates for being primary. In $\S 5$ we prove that they are indeed primary. In $\S \S 6,7,9$, and 10 we prove that there are no other primary orbits. To do this we use some properties of primary orbits which are extensions of other ones. We state and prove these properties in $\S 8$. In $\S 11$ we prove that the existence of some primary orbits implies (or does not imply) the existence of some others. In $\S 12$ we develop the techniques of building examples necessary to prove Main Theorem (b). In $§ 13$ we deduce the Main Theorem from results proved in earlier sections and state some final remarks and conjectures.

Many notions and ideas of this paper can be found in earlier papers (see references). In most cases, they were developed gradually and in parallel by several authors. We shall not try to trace their origin and will use them without referring to earlier papers. For instance, when working on this paper, we were unaware of the papers [Be4, Ba], which contain (for maps of the interval) some ideas very close to ours.

The reader is advised to draw figures when reading most of the proofs, especially in $\S \S 5-11$. We recommend using the corresponding black, blue, green, and red pens.

\section{BASIC DEFINITIONS AND PRELIMINARY RESULTS}

From now on, $X$ will denote indistinctly the interval $[0,1]$ or the space $Y$. Also, $\mathbf{X}$ will denote indistinctly the family $\mathbf{I}$ or the family $\mathbf{Y}$. Depending on the family of maps, $E$ will denote the following: if the family is $I$, then $E=\varnothing$; if the family is $\mathbf{Y}$, then $E=\{0\}$. In such a way, we can say that $\mathbf{X}$ is the family of all continuous maps of $X$ into itself for which $f(E)=E$ (in other words, for which $\left.f\right|_{E}=\left.\operatorname{Id}\right|_{E}$ ). Most of the preliminary definitions and results can be generalized, for example to more complicated trees. To avoid making 
this paper too long (although it is, anyhow), we leave such generalizations to the eager readers.

Let $P$ be a subset of $X$. We shall write $E P$ for $E \cup P$. The set $\operatorname{Span}(P)$ is the smallest connected subset of $X$ containing $P$. If $\operatorname{Span}(P) \subsetneq \operatorname{Span}(Q)$, we say that $P$ has strictly smaller span than $Q$.

The closures of components of $Y \backslash\{0\}$ will be called branches. The meaning of the word interval is obvious for subsets of $[0,1]$. For subsets of $Y$ it will be used in a geometric sense (not a topological one); i.e., an interval has to be contained in one of the branches. When denoting intervals with use of their endpoints, we shall not take into account the ordering; i.e., $[x, y]=[y, x]$. In what follows, in most of the cases, the word interval will mean a closed interval. If not, we say so explicitly and use the standard notation $[x, y)=(y, x]$, $(x, y]=[y, x)$, and $(x, y)=(y, x)$.

If a finite set $P$ is $f$-invariant (i.e., $f(P) \subset P$ ), then the closures of components of $\operatorname{Span}(E P) \backslash E P$ will be called basic intervals (or EP-basic intervals, if confusion may occur). Of course, they are intervals.

If $I$ and $J$ are intervals, we say that $I f$-covers $J k$ times if there are $k$ subintervals of $I$ with pairwise disjoint interiors, each of them mapped onto $J$ by $f$. If $k \geq 1$ but we do not specify it, we say simply that $I f$-covers $J$. Then we shall write simply $I \rightarrow J$ (or put $k$ arrows if $I f$-covers $J k$ times).

If $f \in \mathbf{X}$ and $P$ is a finite $f$-invariant set, then the EP-graph of $f$ is the oriented graph with all basic intervals as vertices and having an arrow from $I$ to $J$ if and only if $I f$-covers $J$. The generalized EP-graph of $f$ has $k$ arrows from $I$ to $J$ if and only if $I f$-covers $J k$ times.

We shall use generalized graphs only once and in a very simple case. To avoid unnecessary complications, we shall not work with generalized graphs in other places.

A loop of length $k$ in an $E P$-graph of $f$ is a sequence of vertices $I_{1}, I_{2}, \ldots$, $I_{k}$ such that $I_{i} f$-covers $I_{i+1}$ for $i=1,2, \ldots, k-1$ and $I_{k} f$-covers $I_{1}$. We shall denote such a loop by $I_{1} \rightarrow I_{2} \rightarrow \cdots \rightarrow I_{k} \rightarrow I_{1}$ and identify it with each of the loops $I_{i} \rightarrow I_{i+1} \rightarrow \cdots \rightarrow I_{k} \rightarrow I_{1} \rightarrow I_{2} \rightarrow \cdots \rightarrow I_{i-1} \rightarrow I_{i}$. When talking about loops we shall use expressions such as "we are moving along an arrow," "we are moving along the loops," and "we are making steps," which have (we hope) obvious meanings.

We say that we add the loop $I_{1} \rightarrow I_{2} \rightarrow \cdots \rightarrow I_{k} \rightarrow I_{1}$ to the loop $J_{1} \rightarrow$ $J_{2} \rightarrow \cdots \rightarrow J_{l} \rightarrow J_{1}$ if they have a common vertex $I_{i}=J_{j}$ and we form a new loop

$$
\begin{aligned}
I_{1} & \rightarrow I_{2} \rightarrow \cdots \rightarrow I_{i} \rightarrow J_{j+1} \rightarrow J_{j+2} \rightarrow \cdots \\
& \rightarrow J_{l} \rightarrow J_{1} \rightarrow \cdots \rightarrow J_{j} \rightarrow I_{i+1} \cdots \rightarrow I_{k} \rightarrow I_{1} .
\end{aligned}
$$

Usually it will be clear which common vertex we use. For adding a loop to itself we shall also use descriptions such as "going along the loop twice" and "taking 
this loop twice," with obvious (again we hope) meaning, or we shall talk about the repetition ( $l$ times) of the loop.

A loop which is not a repetition of a shorter loop will be called nonrepetitive.

A loop which cannot be formed by adding two loops will be called elementary. Note that the loop $I_{1} \rightarrow I_{2} \rightarrow \cdots \rightarrow I_{k} \rightarrow I_{1}$ is elementary if and only if $i \neq j$ implies $I_{i} \neq I_{j}$.

We shall say that an interval $I f$-covers an interval $J$ in $k$ steps if $I f^{k}$ covers $J$. We shall then write $I \underset{k \text { steps }}{\rightarrow} J$.

The following result is not difficult to prove.

Lemma 1.1. Let $I$ and $J$ be intervals. If $f(I) \supset J$ then $I f$-covers $J$.

In $\S \S 1-10$, if $E=\{0\}$, we shall not consider $\{0\}$ as a periodic orbit. Of course, when it comes to proving the Main Theorem we shall have to take it into account again.

If $\alpha=I_{1} \rightarrow I_{2} \rightarrow \cdots \rightarrow I_{k} \rightarrow I_{1}$ is a loop in the $E P$-graph of $f$ and $Q$ is a periodic orbit of $f$, we shall say that $\alpha$ and $Q$ are associated to each other if there exists $x \in Q$ such that $f^{i}(x) \in I_{i+1}$ for $i=0,1, \ldots, k-1$ and $f^{k}(x)=x$.

Remark 1.2. Let $P$ and $Q$ be periodic orbits of $f \in \mathbf{X}$. Assume that $Q$ is associated to a loop $\alpha$ in the $E P$-graph of $f$. Then:

(a) The period of $Q$ divides the length of $\alpha$.

(b) $Q$ is associated to all repetitions of $\alpha$.

Lemma 1.3 [BGMY]. If $f \in \mathbf{X}$ and $\alpha$ is a loop in an EP-graph of $f$, then there exists a periodic orbit $Q$ of $f$, associated to $\alpha$.

Remark 1.4. Obviously, the above definition, remark, and lemma can be applied not only to $E P$-graphs but also to all graphs with intervals as vertices and arrow from $I$ to $J$ if $I f$-covers $J$. In this more general situation, some serious troubles may occur with establishing the period of $Q$. We shall comment on it when this more general version is used.

If $I \subset X$ is an interval and $f \in \mathbf{X}$, we shall say that $f$ is linear on $I$ if $f$ maps $I$ homeomorphically onto its image and $I$ can be divided into subintervals such that each of them is mapped linearly (more precisely affinely) onto its image and the rate of expansion on all these subintervals is the same. Notice that since we work only with $[0,1]$ and $Y$, the number of pieces into which we divide $I$ can be taken smaller than or equal to two (one in the case of $X=[0,1])$.

We shall say that an elementary loop $I_{1} \rightarrow I_{2} \rightarrow \cdots \rightarrow I_{k} \rightarrow I_{1}$ is thin if each $I_{i} f$-covers only one basic interval.

Lemma 1.5. Let $f \in \mathbf{X}$ and let $P$ be a periodic orbit of $f$. Then there is at most one thin loop in the EP-graph of $f$. Moreover, if $\alpha=I_{1} \rightarrow I_{2} \rightarrow \cdots \rightarrow I_{k} \rightarrow I_{1}$ is a thin loop then one of two cases occurs: 
(a) $X=Y, 0$ is one of the endpoints of all $I_{i}$ 's, $P$ has period 1, 2, or 3, and all points of $P$ lie on different branches of $Y$.

(b) The endpoints of all $I_{i}$ 's belong to $P, P$ has period $2 k$, and if $I_{i}=$ $\left[x_{i}, y_{i}\right]$, then $f^{k}\left(x_{i}\right)=y_{i}$ and $f^{k}\left(y_{i}\right)=x_{i}$.

Proof. Assume that $\alpha=I_{1} \rightarrow I_{2} \rightarrow \cdots \rightarrow I_{k} \rightarrow I_{1}$ is a thin loop and $I_{i}=$ $\left[x_{i}, y_{i}\right]$. Since $I_{i} f$-covers only one basic interval, this interval is $\left[f\left(x_{i}\right)\right.$, $\left.f\left(y_{i}\right)\right]$. Hence the set $Z=\left\{x_{1}, x_{2}, \ldots, x_{k}, y_{1}, y_{2}, \ldots, y_{k}\right\}$ is $f$-invariant. Since it contains at least two points, $Z=E P$ or $Z=P$. If $Z=E P$ then obviously case (a) occurs. In this case there is no other elementary loop except $\alpha$.

Assume $Z=P$. The set $P$ contains an extremal point of $\operatorname{Span}(E P)$, i.e., a point such that there is only one basic interval adjacent to it. We may assume that this point is $x_{1}$. Then the adjacent interval is $I_{1}$. We claim that $f^{k}\left(x_{1}\right)=$ $y_{1}$.

Suppose that $f^{k}\left(x_{1}\right)=x_{1}$. Then $f^{j}\left(x_{1}\right)=y_{1}$ for some $j<k$, and $\left[y_{1}, z\right]$, where $z=f^{j}\left(y_{1}\right)$, is the interval $I_{j}$. Since $\alpha$ is elementary, $z \neq x_{1}$. We have $f^{2 k-2 j}(z)=f^{2 k-2 j}\left(f^{2 j}\left(x_{1}\right)\right)=x_{1}$. Therefore, since $I_{1}$ is the only basic interval containing $x_{1}, f^{2 k-2 j}\left(\left[y_{1}, z\right]\right)=I_{1}$. Thus, $f^{2 k-2 j}\left(y_{1}\right)=y_{1}$. This is impossible because $x_{1}, y_{1}$, and $z$ are three points of the same periodic orbit. Hence, indeed, $f^{k}\left(x_{1}\right)=y_{1}$.

Since $f^{k}\left(x_{1}\right)=y_{1}$ and $\alpha$ is a thin loop, we have $f^{k}\left(x_{i}\right)=y_{i}$ and $f^{k}\left(y_{i}\right)=x_{i}$ for all $i$. This follows from the fact that, for each $l, f^{l} \circ f^{k}=f^{k} \circ f^{l}$. From this it follows that $P$ has period $2 k$. Hence, if $Z=P$ then case (b) occurs.

If $\beta$ is another thin loop, then for $\alpha$ and $\beta$ case (a) cannot occur (there is only one elementary loop then). Hence, (b) applies to $\alpha$ and $\beta$. In particular, the length of $\beta$ is also $k$. If $\beta$ goes through some basic interval $J=[w, t]$, then $w=x_{i}$ or $w=y_{i}$ for some $i$. But then $f^{k}(w)=t, f^{k}\left(x_{i}\right)=y_{i}$, and $f^{k}\left(y_{i}\right)=x_{i}$. Hence $J=I_{i}$ and $\alpha=\beta$.

We shall call a thin loop $\alpha$ positive if (a) of Lemma 1.5 occurs and negative if (b) of Lemma 1.5 occurs.

Definition 1.6. Let $f \in \mathbf{X}$ and let $P$ be a periodic orbit of $f$. We shall call $f$ EP-adjusted if:

(i) For every basic interval $[x, y], f$ maps it homeomorphically onto $\operatorname{Span}(\{f(x), f(y)\})$.

(ii) $f(X)=\operatorname{Span}(E P)$.

(iii) If $\alpha=I_{1} \rightarrow I_{2} \rightarrow \cdots \rightarrow I_{k} \rightarrow I_{1}$ is a thin loop then: if $\alpha$ is positive, then $\left.f^{k}\right|_{I_{1}}$ has only the endpoints as fixed points; if $\alpha$ is negative, then $\left.f^{k}\right|_{I_{1}}$ has only the endpoints as periodic points of period 2 .

(iv) If a basic interval does not appear in a thin loop, then $f$ is linear on it. 
Remark 1.7. Let $f \in \mathbf{X}$ be $E P$-adjusted. Take the $R$-graph of $f$, where $R=f^{-n}(E P) \cap \operatorname{Span}(E P)$ (clearly $E \subset R$ and hence $\left.E R=R\right)$. If $\alpha=I_{1} \rightarrow$ $I_{2} \rightarrow \cdots \rightarrow I_{k} \rightarrow I_{1}$ is a thin loop in the $R$-graph of $f$, then each $I_{i}$ is a $E P$-basic interval and $\alpha$ is a thin loop in the $E P$-graph of $f$. Hence, if we replace $E P$ by $R$ in Lemma 1.5, it will remain true.

Lemma 1.8. If $P$ is a periodic orbit of a map $f \in \mathbf{X}$, then there exists a map $g \in \mathbf{X}$ such that $\left.g\right|_{E P}=\left.f\right|_{E P}$ and $g$ is EP-adjusted.

Proof. It is very easy to construct $h \in \mathbf{X}$ such that $\left.h\right|_{E P}=\left.f\right|_{E P}$ and (i), (ii), and (iv) of Definition 1.6 are satisfied. By Lemma 1.5 there is at most one thin loop $\alpha=I_{1} \rightarrow I_{2} \rightarrow \cdots \rightarrow I_{k} \rightarrow I_{1}$ in the $E P$-graph of $h$. We can adjust $h$ (and then call it $g$ ) on $I_{1}$ to satisfy (iii). If $\alpha$ is positive then this is obvious. If $\alpha$ is negative, we choose a homeomorphism $\varphi: I_{1} \rightarrow I_{1}$, reversing orientation and having only endpoints as periodic points of period two. Then we set $\left.g\right|_{I_{1}}=\left(\left.h^{k-1}\right|_{I_{2}}\right)^{-1} \circ \varphi$.

Remark 1.9. From Definition 1.6 it follows immediately that if $\left.f\right|_{E P}=\left.g\right|_{E P}$ and both $f$ and $g$ are $E P$-adjusted, then the $E P$-graphs of $f$ and $g$ are identical. If only $f$ is $E P$-adjusted, then the $E P$-graph of $f$ is a subgraph of the $E P$-graph of $g$.

Proposition 1.10. Let $P$ be a periodic orbit of an EP-adjusted map $f \in \mathbf{X}$. Let $n \geq 0$ and let $R=f^{-n}(E P) \cap \operatorname{Span}(E P)$. Then, for every loop $\alpha$ of the $R$-graph of $f$ one of the following statements holds.

(a) $\alpha$ is an $l$ times $(l \geq 1)$ repetition of a thin loop $\beta$ of length $k$. Then:

(a.1) If $\beta$ is positive, then only $P$ is associated to $\alpha$.

(a.2) If $\beta$ is negative and $l$ is even, then there are two periodic orbits associated to $\alpha: P$ of period $2 k$ and some orbit of period $k$.

(a.3) If $\beta$ is negative and $l$ is odd, then there is only one periodic orbit associated to $\alpha$; this orbit has period $k$.

(b) $\alpha$ is not a repetition of a thin loop. Then there is exactly one periodic orbit associated to $\alpha$.

Moreover, if an orbit associated to $\alpha$ is different from $P$ and its period is smaller than or equal to $n+1$, then $\alpha$ is elementary.

Proof. Statements in case (a) follow from Lemmas 1.3 and 1.5, Remark 1.7, and Definition 1.6(iii). Now assume that $\alpha$ is not a repetition of a thin loop. Then if $\alpha=I_{1} \rightarrow I_{2} \rightarrow \cdots \rightarrow I_{k} \rightarrow I_{1}$, no $I_{i}$ can appear in the thin loop. Hence, by Definition 1.6(i) and (iv), $\left.f^{k}\right|_{I_{1} \cap f^{-1}\left(I_{2}\right) \cap \cdots \cap f^{-k+1}\left(I_{k}\right) \cap f^{-k}\left(I_{1}\right)}$ is linear and expanding and, therefore, it has exactly one fixed point. This proves the statement of (b).

Let $Q$ be a periodic orbit associated to $\alpha$. Denote the period of $Q$ by $k$. Since $Q$ is different from $P$ (and $E$ ) we can find a loop $\gamma=J_{0} \rightarrow J_{1} \rightarrow$ $\cdots \rightarrow J_{k-1} \rightarrow J_{0}$ in the $E P$-graph of $f$ such that for some $x \in Q$ we have $f^{i}(x) \in \operatorname{Int}\left(J_{i}\right)$ for $i=0,1,2, \ldots, k-1$. For each $i$, we have $f^{i}(x) \in \operatorname{Int}\left(K_{i}\right)$, 
where $K_{i}=J_{i} \cap f^{-1}\left(J_{i+1}\right) \cap \cdots \cap f^{-k+1}\left(J_{i+k-1}\right)$, where the addition in the subindices of $J$ is modulo $k$. Each interval $K_{i}$ is an $f^{-k+1}(E P) \cap \operatorname{Span}(E P)$ basic interval. If $K_{i}=K_{j}$ for some $i \neq j$, then the loop $\gamma$ is a repetition of some elementary loop $\beta$ of length smaller than $k$. The orbit which is associated to $\beta$ is also associated to $\gamma$. If $\beta$ is not thin, then by (b) this orbit is equal to $Q$. This contradicts the fact that the period of $Q$ is $k$. If $\beta$ is thin, then we use (a) instead of (b). Since $Q$ is different from $P$, we also obtain a contradiction with the fact that the period of $Q$ is $k$ (we recall that $E$ is not considered a periodic orbit and, hence, $Q \neq E$ ).

Hence $K_{i} \neq K_{j}$ for $i \neq j$. If $k \leq n+1$, each $R$-basic interval is either contained in some $K_{i}$ or disjoint from all $\operatorname{Int}\left(K_{i}\right)$. Therefore it can contain at most one point of $Q$. Consequently $\alpha$ is elementary.

Proposition 1.11. Let $P$ and $Q$ be periodic orbits of an EP-adjusted map $f \in$ $\mathbf{X}$. Assume that $\operatorname{Card}(E P)>1$. Then there is a unique loop in the EP-graph of $f$, associated to $Q$ and of length equal to the period of $Q$. This loop is nonrepetitive unless it is a repetition two times of a thin negative loop and $Q=P$.

Proof. If $Q \neq P$ then the elements of $Q$ belong to the interiors of $E P$-basic intervals. Hence, the existence and uniqueness of the associated loop of length equal to the period of $Q$ are obvious.

Assume that $Q=P$. Consider all pairs $(I, x)$ where $I$ is an $E P$-basic interval and $x$ is one of its endpoints. Let $G$ be the graph with these pairs as vertices and an arrow from $(I, x)$ to $(J, y)$ if $y=f(x)$ and $I f$-covers $J$. Clearly, for each vertex of $G$ there is an arrow beginning at this vertex. If we choose as $x$ an extremal point of $\operatorname{Span}(E P)$ different from 0 , i.e., a point $z \in P$ such that there is only one basic interval $K$ adjacent to it, then we obtain a loop $\alpha$ in $G$ going through $(K, z)$, of length equal to $m$, the period of $P$. Clearly $\alpha$ gives a loop of the same length in the $E P$-graph of $f$, associated to $P$.

Each vertex of $G$ is a beginning of only one arrow. Therefore, any loop in $G$ going through $(K, z)$ has to be equal to $\alpha$ repeated $l \geq 1$ times. Let $\beta=I_{0} \rightarrow I_{1} \rightarrow \cdots \rightarrow I_{m-1} \rightarrow I_{0}$ be a loop in the $E P$-graph of $f$, associated to $P$ where $m$ is the period of $P$. Then there exists $x \in P$ such that $f^{i}(x) \in I_{i}$ for $i=0,1,2, \ldots, m-1$. By the definition of $G,\left(I_{0}, x\right) \rightarrow\left(I_{1}, f(x)\right) \rightarrow$ $\cdots \rightarrow\left(I_{m-1}, f^{m-1}(x)\right) \rightarrow\left(I_{0}, x\right)$ is a loop in $G$. Since one of $f^{i}(x)$ is equal to $z$, this loop has to be equal to $\alpha$. This proves uniqueness in the case $Q=P$.

Now let $\alpha$ be the loop in the $E P$-graph of $f$ associated to $Q$ and of length equal to the period of $Q$. Assume that $\alpha$ is a repetition $l$ times, with $l \geq 2$, of some loop $\beta$ of smaller length. The periodic orbit associated to $\beta$ is also associated to $\alpha$. By Proposition 1.10 (for $n=0$ ), it is $Q$ unless $\beta$ is thin positive or $\beta$ is thin negative and $l$ is even. If the periodic orbit associated to $\beta$ is $Q$, then we obtain a contradiction because the period of $Q$ is larger than the length of $\beta$. If $\beta$ is thin and positive, then $Q=P$ (we recall that $E$ is 
not a periodic orbit and, hence, $Q \neq E$ ). Therefore, the period of $Q$ is equal to the length of $\beta$, which is a contradiction. Hence $\beta$ has to be thin negative and $l$ equals two. Moreover, since the period of $Q$ is larger than the length of $\beta, Q=P$.

Lemma 1.12. Let $f \in \mathbf{X} E P$-adjusted and let $I$ and $J$ be intervals such that $I$ and $J$ are unions of basic intervals and $I$ covers $J$ in $k$ steps. Then for every basic interval $I_{k} \subset J$ there are basic intervals $I_{0}, I_{1}, \ldots, I_{k-1}$ such that $I_{0} \subset I$ and $I_{0} \rightarrow I_{1} \rightarrow \cdots \rightarrow I_{k-1} \rightarrow I_{k}$.

Proof. There is a subinterval $K_{k}$ of $I$ which is mapped by $f^{k}$ onto $I_{k}$ and such that no interior point of $K_{k}$ is mapped to an endpoint of $I_{k}$. Since $E P$ is $f$-invariant, none of the intervals $K_{k}, f\left(K_{k}\right), \cdots, f^{k-1}\left(K_{k}\right)$ can have points of $E P$ in its interior. Denote by $I_{i}$ the basic interval containing $f^{i}\left(K_{k}\right)$ for $i=0,1,2, \ldots, k-1$. Since $f$ is $E P$-adjusted and $E P$ is $f$-invariant, $f\left(I_{i}\right) \supset I_{i+1}$. By Lemma 1.1, $I_{i} f$-covers $I_{i+1}$.

Definition 1.13. Let $f, g \in \mathbf{X}$ and let $P$ be a periodic orbit of $f$ and $Q$ a periodic orbit of $g$. Then $P$ and $Q$ are $E$-equivalent if there exists a homeomorphism $h$ of $\operatorname{Span}(E P)$ onto $\operatorname{Span}(E Q)$ such that $\left.h\right|_{E}=\left.\operatorname{Id}\right|_{E}, h(P)=Q$, and $h \circ f=g \circ h$ on $P$.

Clearly the above relation is an equivalence relation.

Remark 1.14. If $f \in \mathbf{X}$ is $E P$-adjusted, $g \in \mathbf{X}$ is $E Q$-adjusted, and $P$ and $Q$ are $E$-equivalent, then $h$ induces an isomorphism $h^{*}$ of the $E P$-graph of $f$ to the $E Q$-graph of $g$ (i.e., $h^{*}$ is a bijective map from the set of vertices of the $E P$-graph of $f$ to the set of vertices of the $E Q$-graph of $g$ such that, if $I$ and $J$ are vertices of the $E P$-graph of $f$, then $I f$-covers $J$ if and only if $h^{*}(I) f$-covers $\left.h^{*}(J)\right)$. If only $f$ is $E P$-adjusted, $h$ induces an isomorphism of the $E P$-graph of $f$ onto some subgraph of the $E Q$-graph of $g$.

Lemma 1.15. Assume that $f, g \in \mathbf{X}, P$ is a periodic orbit of $f, Q$ is a periodic orbit of $g, P$ and $Q$ are E-equivalent, and $f$ is EP-adjusted. Then for every $n \geq 0$ there exists a homeomorphism $h_{n}$ of $\operatorname{Span}(E P)$ onto $\operatorname{Span}(E Q)$ such that $h_{n \mid E}=\left.\operatorname{Id}\right|_{E}, h_{n}(P)=Q$, and $h_{n} \circ f=g \circ h_{n}$ on $f^{-n}(E P) \cap \operatorname{Span}(E P)$.

Proof. We use induction. For $n=0$ it follows from the definition of $E$ equivalence. Assume that the statement is proved for $n-1$. We set $h_{n}=h_{n-1}$ on $f^{-(n-1)}(E P) \cap \operatorname{Span}(E P)$, and then we have to define $h_{n}$ on all intervals of the partition of $\operatorname{Span}(E P)$ by the points of $f^{-(n-1)}(E P)$. Let $I=[x, y]$ be one of these intervals. Since $I$ is contained in some $E P$-basic interval and $f$ is $E P$ adjusted, $I$ is mapped by $f$ homeomorphically onto $J=\operatorname{Span}(\{f(x), f(y)\})$. The points of $f^{-n}(E P) \cap I$ are inverse images of the points of $f^{-(n-1)}(E P) \cap J$ by $f$ (see Figure 1.16). Now fix some homeomorphism $h:[0,1] \rightarrow h_{n-1}(I)$. By the induction hypothesis,

$$
g \circ h(\{0,1\})=g\left(\left\{h_{n-1}(x), h_{n-1}(y)\right\}\right)=\left\{h_{n-1}(f(x)), h_{n-1}(f(y))\right\} .
$$


For each point $z \in f^{-n}(E P) \cap \operatorname{Int}(I)$, we set

$$
h_{n}(z)=h\left(\inf \left\{t \in[0,1]: g(h(t))=h_{n-1}(f(z))\right\}\right) .
$$

Since $\left.h_{n-1} \circ f\right|_{I}$ is a homeomorphism of $I$ onto

$$
\operatorname{Span}\left(\left\{h_{n-1}(f(x)), h_{n-1}(f(y))\right\}\right),
$$

$h_{n}$ can be extended to a homeomorphism of the whole $I$ onto $h_{n-1}(I)$. For each $z \in f^{-n}(E P) \cap \operatorname{Int}(I)$ we have $g\left(h_{n}(z)\right)=g(h(t))=h_{n-1}(f(z))=$ $h_{n}(f(z))$ because $f(z) \in f^{-(n-1)}(E P)$, where $t$ is given in the definition of $h_{n}(z)$. Hence we have $h_{n} \circ f=g \circ h_{n}$ on $f^{-n}(E P) \cap \operatorname{Int}(I)$. If we make the above construction on each $I$, then we obtain $h_{n}$ satisfying our conditions.

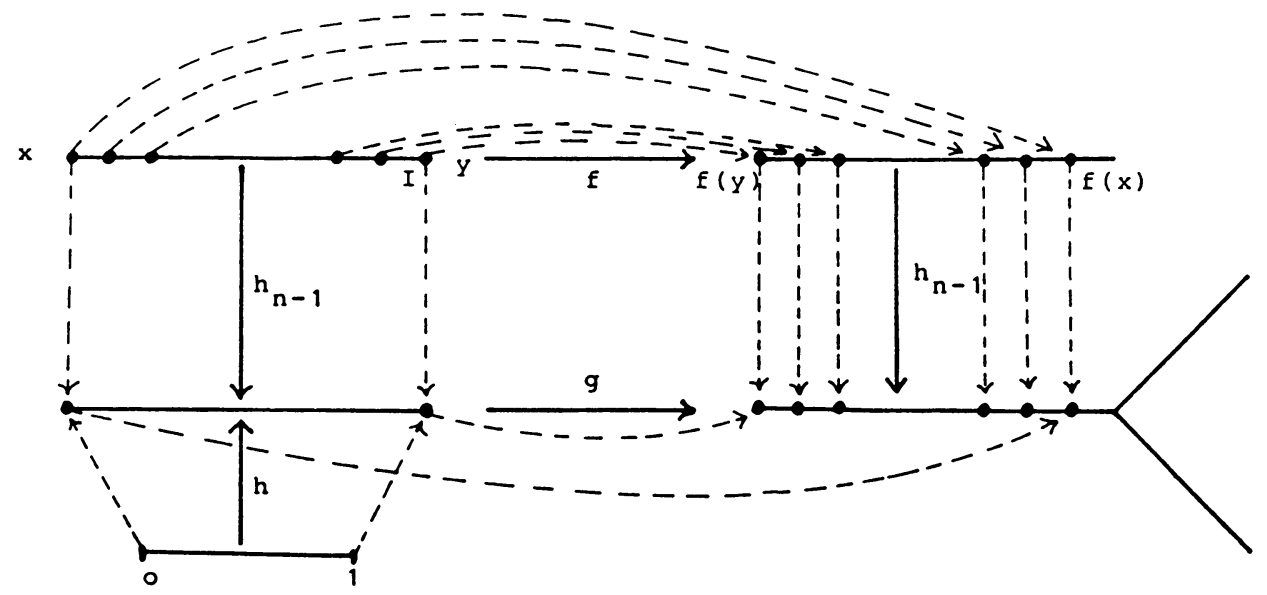

FIGURE 1.16

Remark 1.17. Clearly, Remark 1.14 also holds if we replace $E P$-graph by $f^{-n}(E P) \cap \operatorname{Span}(E P)$-graph, $E Q$-graph by $h_{n}\left(f^{-n}(E P) \cap \operatorname{Span}(E P)\right)$-graph, and $h$ by $h_{n}$, where $h_{n}$ is as in Lemma 1.15 .

The following lemma plays a basic role in the rest of the paper. It allows us to work mainly with adjusted maps.

Lemma 1.18 (Adjusting Lemma). Assume that $f, g \in \mathbf{X}, P$ is a periodic orbit of $f, Q$ is a periodic orbit of $g, P$ and $Q$ are E-equivalent, $f$ is EP-adjusted, and $f$ has a periodic orbit $P^{\prime} \neq P$. Then $g$ has a periodic orbit $Q^{\prime} \neq Q$, $E$-equivalent to $P^{\prime}$ and such that $\operatorname{Span}\left(Q^{\prime}\right) \subsetneq \operatorname{Span}(Q)$.

Proof. Denote the period of $P^{\prime}$ by $n$. Let $h_{n}$ be the homeomorphism obtained from Lemma 1.15. Since $f$ is $E P$-adjusted, by Remark 1.17, $h_{n}$ induces an isomorphism of the $R$-graph of $f$ and some subgraph of the $h_{n}(R)$-graph of $g$, where $R=f^{-n}(E P) \cap \operatorname{Span}(E P)$. Clearly, $P^{\prime}$ is associated to some loop $\alpha$ of length $n$ in the $R$-graph of $f$. Let $\beta$ be the corresponding loop in the 
$h_{n}(R)$-graph of $g$. By Proposition 1.10, $\alpha$ is elementary and therefore $\beta$ is also elementary. Let $Q^{\prime}$ be a periodic orbit of $g$ associated to $\beta$. If $Q^{\prime}=Q$ then $P$ is associated to $\alpha$. Since $P^{\prime} \neq P$ and $P^{\prime} \neq E$ (we recall that $E$ is not considered as a periodic orbit), by Proposition 1.10 we obtain that the period of $P$ is twice the period of $P^{\prime}$, which is strictly smaller than the length of $\alpha$. This is a contradiction since the period of $P^{\prime}$ is equal to the length of $\alpha$. Hence $Q^{\prime} \neq Q$. Clearly, we then have $\operatorname{Span}\left(Q^{\prime}\right) \subsetneq \operatorname{Span}(Q)$. To show that $P^{\prime}$ and $Q^{\prime}$ are $E$-equivalent, we can obtain the homeomorphism required by the definition of $E$-equivalence by modifying $h_{n}$. This can be done because $\alpha$ and $\beta$ are elementary and then in each $R$-basic interval there is at most one point of $P^{\prime}$ and in each $h_{n}(R)$-basic interval there is at most one point of $Q^{\prime}$.

\section{PRIMARY ORBITS}

First we recall the notion of primary orbit.

Definition 2.1. Let $P$ be a periodic orbit of a map $f \in \mathbf{X} . P$ will be called primary if there exists $g \in \mathbf{X}$ such that $\left.g\right|_{E P}=\left.f\right|_{E P}$ and there is no other periodic orbit of $g$ of the same period as $P$. P will be called secondary if it is not primary.

Remark 2.2. If $P$ is a periodic orbit of $f \in \mathbf{X}$ and of $g \in \mathbf{X}$, then whether $P$ is primary or secondary does not depend on whether we regard it as an orbit of $f$ or $g$.

Theorem 2.3 (First Theorem). Assume that $P$ is a periodic orbit of period $m>1$ of an EP-adjusted map $f \in \mathbf{X}$. Let $G$ be the EP-graph of $f$. Then the following conditions are equivalent:

(a) $P$ is primary.

(b) $P$ is the unique periodic orbit of $f$ of period $m$.

(c) Either there is a thin negative loop in $G$ and there is no nonrepetitive loop of length $m$ in $G$, or there is no thin negative loop in $G$ and there is at most one nonrepetitive loop of length $m$ in $G$.

(d) Either there is a thin negative loop in $G$, the repetition two times of this loop is associated to $P$, and there is no nonrepetitive loop of length $m$ in $G$, or there is no thin negative loop in $G$, there is exactly one nonrepetitive loop of length $m$ in $G$, and this loop is associated to $P$.

Proof. By Definition 2.1, (b) implies (a).

Assume that (a) holds but (c) does not. By Proposition 1.11 there is a loop $\alpha$ in the $E P$-graph of $f$, of length $m$, associated to $P$. This loop is unique and nonrepetitive unless it is a repetition two times of a thin negative loop. If there is a thin negative loop $\beta$ in $G$ then, by Proposition 1.10, $P$ is associated to $\beta$ repeated two times. Hence, by Proposition 1.11, $P$ is not associated to any nonrepetitive loop of length $m$. Therefore, since (c) does not hold, in all cases (independent of the existence of the thin negative loop) we obtain the existence 
of a nonrepetitive loop $\gamma \neq \alpha$ in $G$, of length $m$ and not associated to $P$. Let $Q$ be the periodic orbit associated to $\gamma$. By (a), its period is smaller than $m$. Since $\gamma$ is nonrepetitive of length $m$, this gives a contradiction. Therefore (a) implies (c).

Assume that (c) holds. If there is a thin negative loop in $G$ then, by Proposition 1.10 , its repetition two times is associated to $P$ and (d) holds. If there is no such loop in $G$ then, by Proposition 1.11, there is a nonrepetitive loop of length $m$ associated to $P$, and (d) also holds. Therefore (c) implies (d).

Assume that (d) holds but (b) does not. Then $f$ has a periodic orbit $Q \neq P$ of period $m$. By Proposition 1.11, $Q$ is associated to a nonrepetitive loop $\alpha$ of period $m$. By (d), there is no thin negative loop in $G$ and $\alpha$ is associated to $P$. In view of Proposition $1.10(\mathrm{~b})$, this is a contradiction. Therefore (d) implies (b).

Lemma 2.4. A periodic orbit E-equivalent to a primary orbit is primary.

Proof. Let $P$ be a periodic orbit of a map $f \in \mathbf{X}, E$-equivalent to a periodic orbit $Q$ of a map $g \in \mathbf{X}$. Assume that $P$ is primary. By Lemma 1.8 , there are maps $f^{\prime}, g^{\prime} \in \mathbf{X}$ such that $f^{\prime}$ is $E P$-adjusted, $g^{\prime}$ is $E Q$-adjusted, $\left.f^{\prime}\right|_{E P}=$ $\left.f\right|_{E P}$, and $\left.g^{\prime}\right|_{E Q}=\left.g\right|_{E Q}$. By Remark 1.14, the EP-graph of $f^{\prime}$ and the $E Q$ graph of $g^{\prime}$ are isomorphic. By using the First Theorem (2.3) for both $f^{\prime}$ and $g^{\prime}$, it follows that, if $P$ is primary, so is $Q$.

Theorem 2.5 (Primary Theorem). If a map $f \in \mathbf{X}$ has a periodic orbit $P$ of period $m$, then $f$ has a primary periodic orbit of period $m$ with span contained in $\operatorname{Span}(P)$.

Proof. Let $P$ be a periodic orbit of $f$ of period $m$. By Lemma 1.8, there exists an $E P$-adjusted map $g$ such that $\left.g\right|_{E P}=\left.f\right|_{E P}$. The $E P$-graph of $g$ has only a finite number of loops of length $m$. By Propositions 1.10 and 1.11 , $g$ has only a finite number of periodic orbits of period $m$. Hence, $g$ has a periodic orbit $Q$ of period $m$ such that there is no periodic orbit $Q^{\prime}$ of $g$ of period $m$ with $\operatorname{Span}\left(Q^{\prime}\right) \subsetneq \operatorname{Span}(Q)$. Let $h$ be an $E Q$-adjusted map. If $h$ has another orbit of period $m$ then, by the Adjusting Lemma (1.18), $g$ has a periodic orbit $Q^{\prime}, E$-equivalent to this orbit (and consequently of period $m$ ) with $\operatorname{Span}\left(Q^{\prime}\right) \subsetneq \operatorname{Span}(Q)$, a contradiction. Hence, $h$ has no such orbit and, by the First Theorem (2.3), $Q$ is primary. By the Adjusting Lemma, $f$ has a periodic orbit $E$-equivalent to $Q$ with span contained in $\operatorname{Span}(P)$. Clearly this orbit has period $m$. By Lemma 2.4 this orbit is primary.

\section{EXTENSIONS}

In this section we assume that $R$ is a periodic orbit of period $n$ of a map $g \in \mathbf{I}$ and that $Q$ is a periodic orbit of period $s>1$ of a map $h \in \mathbf{X}$. 
Definition 3.1. We shall call a periodic orbit $P$ of period $s . n$ of a map $f \in \mathbf{X}$ an $n$-extension of $Q$, if $P$ can be divided into subsets $P_{0}, P_{1}, \ldots, P_{s-1}$ of cardinality $n$ each such that:

(i) $\operatorname{Span}\left(P_{i}\right) \cap E=\varnothing, i=0,1,2, \ldots, s-1$.

(ii) $\operatorname{Span}\left(P_{i}\right) \cap \operatorname{Span}\left(P_{j}\right)=\varnothing$ for $i \neq j$.

(iii) There is a map $\psi:\{0, \ldots, s-1\} \rightarrow\{0, \ldots, s-1\}$ such that if $x \in P_{i}$ then $f(x) \in P_{\psi(i)}$.

(iv) If we collapse each $\operatorname{Span}\left(P_{i}\right)$ to a point then the periodic orbit $\tilde{P}$ (obtained from $P$ ) of $\tilde{f}$ (obtained from $f$ ) is $E$-equivalent to $Q$.

This definition perhaps needs some comments:

1. Let $\pi$ be the map $X \rightarrow \tilde{X}$ which collapses each $\operatorname{Span}\left(P_{i}\right)$ to a point (call this point $y_{i}$ ). Then $\tilde{f}$ can be defined in the following way:

$$
\tilde{f}(x)= \begin{cases}\pi \circ f \circ \pi^{-1}(x) & \text { if } x \notin\left\{y_{0}, y_{1}, \ldots, y_{s-1}\right\}, \\ y_{\psi(i)} & \text { if } x=y_{i} .\end{cases}
$$

By (iii) and the continuity of $f, \tilde{f}$ is well defined and continuous. $\tilde{P}=$ $\left\{y_{0}, y_{1}, \ldots, y_{s-1}\right\}$ is a periodic orbit of $\tilde{f}$ of period $s$. Also $\tilde{X}$ is homeomorphic to $X$. Hence, (iv) makes sense.

2. Notice that by (iii), all $P_{i}$ are periodic orbits of period $n$ of $f^{s}$. If $f$ is $E P$-adjusted, then $\tilde{f}$ is $E \tilde{P}$-adjusted (for a suitable choice of a homeomorphism between $\tilde{X}$ and $X$; remember that the definition of adjusted maps was for maps of $X$, not of $\tilde{X}), \tilde{f} \circ \pi=\pi \circ f$, and the set $\bigcup_{i=0}^{s-1} \operatorname{Span}\left(P_{i}\right)$ is $f$-invariant.

Remark 3.2. For given $n$ and $Q$ it is very easy to construct an $n$-extension of $Q$.

Definition 3.3. We shall call a periodic orbit $P$ of period $s n$ of a continuous map $f \in \mathbf{Y}$ an $R$-extension of $Q$, if $P$ is an $n$-extension of $Q$ and

(v) The periodic orbit $P_{0}$ of $f^{s}$ is $E$-equivalent to $R$.

(vi) On all $P_{i}$, except at most one, $f$ is monotone.

Lemma 3.4. Under assumptions (i), (ii), (iii), and (vi), condition (v) is equivalent to

$\left(\mathrm{v}^{\prime}\right)$ The periodic orbit $P_{i}$ of $f^{s}$ is E-equivalent to $R$ for each $i$.

Proof. Obviously (v) follows from ( $\left.\mathrm{v}^{\prime}\right)$. Assume (i), (ii), (iii), (v), and (vi) and prove $\left(\mathrm{v}^{\prime}\right)$. Without loss of generality we can assume that $\psi(i)=i+1$ $(\bmod s)$. By $(v i)$, there is some $j \in\{0,1, \ldots, s-1\}$ such that $f$ is monotone on all $P_{i}$ for $i \neq j$. Hence, $f^{i}$ is monotone on $P_{0}$ for $i=1,2, \ldots, j$ and $f^{s-k}$ is monotone on $P_{k}$ for $k=j+1, \ldots, s-1$. The maps $\left.f^{i}\right|_{P_{0}}$ for $i=1,2, \ldots, j$ and $\left.f^{s-k}\right|_{P_{k}}$ for $k=j+1, \ldots, s-1$ can be extended to homeomorphisms of $\operatorname{Span}\left(P_{0}\right)$ onto $\operatorname{Span}\left(P_{j}\right)$ for $i=1,2, \ldots, j$ and of $\operatorname{Span}\left(P_{k}\right)$ onto $\operatorname{Span}\left(P_{0}\right)$ for $k=j+1, \ldots, s-1$, respectively. Since iterates of $f$ commute with one another, these homeomorphisms give equivalence of 
$P_{0}$ and $P_{i}$ for $i=1, \ldots, j, j+1, \ldots, s-1$. By (v), $P_{0}$ is $E$-equivalent to $R$, and hence all $P_{i}$ are $E$-equivalent to $R$.

Remark 3.5. For given orbits $R$ and $Q$, it is very easy to construct an $R$ extension of $Q$.

Remark 3.6. If $P$ is a 2-extension of $Q$ then $P$ is an $R$-extension of $Q$ for each periodic orbit $R$ of period two (this follows from the fact that each map on two points is monotone). Hence, for 2-extensions we may use results with $R$-extensions in the hypotheses. Notice also that if $f$ is $E P$-adjusted, then the existence of a thin negative loop in the $E P$-graph of $f$ is equivalent to $P$ being a 2-extension of some orbit.

Remark 3.7. In Definitions 3.1 and 3.3 we did not use $g$ and $h$ outside $R$ and $Q$, respectively. Hence, we may assume that $g$ is $R$-adjusted and $h$ is $E Q$-adjusted.

In the next lemmas we shall use the notation of Definitions 3.1 and 3.3.

Lemma 3.8. Let $P$ be an R-extension of $Q$. Assume that $f$ is EP-adjusted and that $f$ has a periodic orbit $P^{\prime} \neq P$. Then either $P^{\prime}$ is an $R^{\prime}$-extension of $Q$ for some periodic orbit $R^{\prime} \neq R$ of $g$, or $P^{\prime}$ is E-equivalent to some periodic orbit $Q^{\prime} \neq Q$ of $h$.

Proof. Since $\bigcup_{i=0}^{s-1} \operatorname{Span}\left(P_{i}\right)$ is $f$-invariant, there are two possibilities:

1. $P^{\prime} \subset \bigcup_{i=0}^{s-1} \operatorname{Span}\left(P_{i}\right)$. Denote $P_{i}^{\prime}=P^{\prime} \cap \operatorname{Span}\left(P_{i}\right)$. Since $f$ is $E P$ adjusted, $\operatorname{Span}\left(P_{0}\right)$ is $f^{s}$-invariant, and (vi) holds, we have that $\left.f^{s}\right|_{\operatorname{Span}\left(P_{0}\right)}$ is $P_{0}$-adjusted. Since $P^{\prime} \neq P$, we have $P_{0}^{\prime} \neq P_{0}$. Therefore, by the Adjusting Lemma (1.18) $g$ has a periodic orbit $R^{\prime} \neq R \quad E$-equivalent to $P_{0}^{\prime}$. In view of Lemma 3.4, $P^{\prime}$ is an $R^{\prime}$-extension of $Q$.

2. $P^{\prime} \cap\left(\bigcup_{i=0}^{s-1} \operatorname{Span}\left(P_{i}\right)\right)=\varnothing$. Then the orbit $\tilde{P}^{\prime}=\pi\left(P^{\prime}\right)$ of $\tilde{f}$ obtained from $P^{\prime}$ by collapsing each $\operatorname{Span}\left(P_{i}\right)$ to a point is $E$-equivalent to $P^{\prime}$. Since $f$ is $E P$-adjusted, $\tilde{f}$ is $E \tilde{P}$-adjusted. Clearly, $\tilde{P}^{\prime} \neq \tilde{P}$. By (iv) and the Adjusting Lemma, $h$ has a periodic orbit $Q^{\prime} \neq Q \quad E$-equivalent to $\tilde{P}^{\prime}$. Then $P^{\prime}$ is $E$-equivalent to $Q^{\prime}$.

Lemma 3.9. Let $P$ be an $n$-extension of $Q$. Assume that $f$ is EP-adjusted and that for some $i,\left.f^{s}\right|_{\operatorname{Span}\left(P_{i}\right)}$ has a periodic orbit $P_{i}^{\prime} \neq P_{i}$. Then $f$ has a periodic orbit $P^{\prime} \neq P$ of period s. $\operatorname{Card}\left(P_{i}^{\prime}\right)$.

Proof. Since $f$ is $E P$-adjusted, we have $f^{j}\left(P_{i}^{\prime}\right) \subset \operatorname{Span}\left(P_{\psi^{j}(i)}\right)$ for each $j(\psi$ is from (iii) of Definition 3.1). Hence, $P^{\prime}=\bigcup_{j=0}^{s-1} f^{j}\left(P_{i}^{\prime}\right)$ is a periodic orbit of $f$ of period $\operatorname{Card}\left(P_{i}^{\prime}\right) s$ and $P^{\prime} \neq P$.

Lemma 3.10. Let $P$ be an $R$-extension of $Q$. Assume that $g$ is $R$-adjusted and has a periodic orbit $R^{\prime} \neq R$. Then $f$ has a periodic orbit $P^{\prime} \neq P$ of period $\operatorname{Card}\left(R^{\prime}\right) . s$. 
Proof. In view of the Adjusting Lemma (1.18), we may assume that $f$ is $E P$ adjusted. Then, again by the Adjusting Lemma, $\left.f^{s}\right|_{\operatorname{Span}\left(P_{0}\right)}$ has a periodic orbit $P_{0}^{\prime} \neq P_{0}$, equivalent to $R^{\prime}$. By Lemma 3.9, $f$ has a periodic orbit $P^{\prime} \neq P$ of period $\operatorname{Card}\left(R^{\prime}\right) s$.

Lemma 3.11. Let $P$ be an n-extension of $Q$. Then $f$ has a periodic orbit $E$-equivalent to $Q$. Moreover, if $h$ is EQ-adjusted and has a periodic orbit $Q^{\prime} \neq Q$, then $f$ has a periodic orbit $P^{\prime} \neq P$, E-equivalent to $Q^{\prime}$.

Proof. Since $\operatorname{Span}\left(P_{i}\right) f$-covers $\operatorname{Span}\left(P_{\psi(i)}\right)$ for each $i, f$ has a periodic orbit $E$-equivalent to $Q$. Since $h$ is $E Q$-adjusted and (iv) holds, by the Adjusting Lemma (1.18), $\tilde{f}$ has a periodic orbit $\tilde{P}^{\prime} \neq \tilde{P} \quad E$-equivalent to $Q^{\prime}$. The orbit $\tilde{P}^{\prime}$ is an image of some periodic orbit $P^{\prime}$ of $f$ under $\pi$. Clearly, $P^{\prime} \neq P$ and $P^{\prime}$ is $E$-equivalent to $P^{\prime}$. Hence $P^{\prime}$ is $E$-equivalent to $Q^{\prime}$.

Lemma 3.12. Let $P$ be an R-extension of $Q$. If $h$ has no periodic orbits of period $m=\operatorname{Card}(P)$ and $R$ is primary, then $P$ is primary.

Proof. We may assume that $f$ is $E P$-adjusted and $g$ is $R$-adjusted. Suppose that $P$ is secondary. Then $f$ has a periodic orbit $P^{\prime} \neq P$ of period $m$. Since $h$ has no periodic orbits of period $m$, by Lemma 3.8, $P^{\prime}$ is an $R^{\prime}$-extension of $Q$ for some periodic orbit $R^{\prime} \neq R$ of $g$. Since $g$ is $R$-adjusted, $R$ is primary, and periods of $R^{\prime}$ and $R$ are equal, in view of the First Theorem (2.3), we obtain a contradiction.

Lemma 3.13. Let $P$ be an n-extension of $Q$. If $P$ is primary, then $P_{i}$ is a primary orbit of $f^{s}$ for each $i$. If additionally $h$ is EQ-adjusted, then $h$ has no periodic orbit $Q^{\prime} \neq Q$ of period $m \equiv \operatorname{Card}(P)$.

Proof. We may assume that $f$ is $E P$-adjusted. Suppose that, for some $i$, the orbit $P_{i}$ of $f^{s}$ is secondary. Since $\operatorname{Span}\left(P_{i}\right)$ is $f^{s}$-invariant, $f^{s}$ has a periodic orbit $P_{i}^{\prime} \subset \operatorname{Span}\left(P_{i}\right)$, of the same period as $P_{i}$ and different from $P_{i}$. By Lemma $3.9 f$ has a periodic orbit $P^{\prime} \neq P$ of period $m$. Since $P$ is primary and $f$ is $E P$-adjusted, from the First Theorem (2.3), we get a contradiction.

Let us now make the additional assumption that $h$ is $E Q$-adjusted. Suppose that $h$ has a periodic orbit $Q^{\prime} \neq Q$ of period $m$. By Lemma 3.11, $f$ has a periodic orbit $P^{\prime} \neq P, E$-equivalent to $Q^{\prime}$. The period of $P^{\prime}$ is $m, P$ is primary, and $f$ is $E P$-adjusted, a contradiction.

\section{CANDIDATES FOR PRIMARY ORBITS}

We denote the branches of $Y$ by $\mathrm{br}_{0}, \mathrm{br}_{1}, \mathrm{br}_{2}$. Since we shall adjust this notation to the periodic orbit under consideration, we do not specify which branch has which number.

For $x \in Y \backslash\{0\}$ we define the index of $x$ by $\operatorname{ind}(x)=i$ if and only if $x \in \mathrm{br}_{i}$. We shall write $x \sim y$ if and only if $\operatorname{ind}(x)=\operatorname{ind}(y)$.

If $x=0$ or $x \sim y$ and $x \in[0, y)$, we say that $x$ is smaller than $y$ and write $x<y$. Analogously, we define $>, \leq, \geq$. 
If $P$ is a periodic orbit of $f \in \mathbf{X}$ and $x \in P$, then we call the ordered pair $A=(x, f(x))$ an arrow with the beginning $b(A)=x$ and end $e(A)=f(x)$.

If $f \in \mathbf{Y}$, then the direction of an arrow $A$ is a number $\operatorname{dir}(A) \in\{-1,0,1\}$ such that $\operatorname{dir}(A) \equiv \operatorname{ind}(e(A))-\operatorname{ind}(b(A))$ (we recall that the symbol $\equiv$ denotes congruency modulo 3 ).

If $P$ has at least one point on a branch $\mathrm{br}_{i}$, then we denote by $\mathrm{sm}_{i}$ the smallest point of $P$ on this branch (remember that if $f \in Y$ we always assume that $\{0\}$ is not a periodic orbit of $f$ ). The arrow beginning at $\mathrm{sm}_{i}$ will be denoted by $\operatorname{sm} A_{i}$. We shall call the arrows $\operatorname{sm} A_{i}$ the smallest arrows.

A periodic orbit $P$ of $f \in \mathbf{Y}$ will be called directed if $P$ has at least one point on each branch and the direction of the three smallest arrows is the same and different from 0 . Otherwise we call $P$ undirected.

We consider the undirected case first. In a parallel way, we shall make the same definitions for maps of an interval. Hence, our assumptions now are $f \in \mathbf{X}, P$ is a periodic orbit of $f$ of period $m$, and, if $f \in \mathbf{Y}$, then $P$ is undirected. We shall distinguish five cases:

(I) $f \in \mathbf{I}$.

(II) $f \in \mathbf{Y}, P$ lies on one branch.

(III) $f \in \mathbf{Y}, P$ lies on two branches.

(IV) $f \in \mathbf{Y}, P$ lies on three branches and all smallest arrows have direction different from 0 .

(V) $f \in \mathbf{Y}, P$ lies on three branches, and some of the smallest arrows have direction 0 .

We have to introduce some new notation. In case III, we call one of the branches on which $P$ lies odd and the other one even. In case IV, since not all smallest arrows have the same direction, there are two of them ending on the same branch. We call this branch odd and the other two branches even.

With the above definitions, in cases III and IV we can talk about the parity of branches on which elements of $P$ lie.

In cases III and IV an arrow $A$ will be called black if $b(A)$ and $e(A)$ lie on branches of different parity and blue otherwise.

Definition 4.1. We define a pendulum orbit as follows (see Figures 4.2-4.16). For $m=1$. The case is I or II. In this case $P$ is always pendulum (however, we have to remember that if $f \in \mathbf{Y}$ we do not consider $\{0\}$ as a periodic orbit). For $m>1, m$ odd. In cases III and IV we say that $P$ is pendulum if:

(i) $P$ has exactly one blue arrow $A$.

(ii) If we denote $x_{i}=f^{i}(e(A)), i=0,1,2, \ldots, m-1$, then if $x_{i} \sim x_{j}$, $x_{i}<x_{j}$ is equivalent to $i<j$.

In cases I and II we say that $P$ is pendulum if $f$ has a fixed point $e \in$ $\operatorname{Span}(P)$ such that:

(i) $P$ has exactly one arrow $A$ with $b(A)$ and $e(A)$ on the same side of $e$. 
(ii) If we denote $x_{i}=f^{i}(e(A)), i=0,1, \ldots, m-1$, then for $x_{i}, x_{j}$ lying on the same side of $e, x_{i} \in\left(e, x_{j}\right)$ is equivalent to $i<j$ (remember that $\left.\left(e, x_{j}\right)=\left(x_{j}, e\right)\right)$.

In case $\mathrm{V} P$ is not pendulum.

Notice that in case IV we have $m \geq 5$. Notice also that in cases I and II we have either

$$
\begin{gathered}
x_{m-1}<x_{m-3}<\cdots<x_{2}<x_{0}<e<x_{1}<x_{3}<\cdots<x_{m-4}<x_{m-2} \text { or } \\
x_{m-1}>x_{m-3}>\cdots>x_{2}>x_{0}>e>x_{1}>x_{3}>\cdots>x_{m-4}>x_{m-2} .
\end{gathered}
$$

In case III we have

$$
0<x_{0}<x_{2}<\cdots<x_{m-3}<x_{m-1} \text { on one branch }
$$

and

$$
0<x_{1}<x_{3}<\cdots<x_{m-4}<x_{m-2} \text { on another branch. }
$$

For $m$ even. In cases I, II, and III we divide the definition into two steps:

(1) If $m=2^{k}, k \geq 1$, we use induction. If $k=1, P$ is always pendulum. If $k>1$ and pendulum orbits of period $2^{k-1}$ are defined, then $P$ is pendulum if it is a 2-extension of a pendulum orbit of period $2^{k-1}$.

(2) If $m=2^{k} . n, k \geq 1, n$ is odd, and $n \geq 3$, then $P$ is pendulum if $P$ is an $R$-extension of a pendulum orbit of period $2^{k}$, and $R$ is a pendulum orbit of period $n$.

In cases IV and V, $P$ is not pendulum.

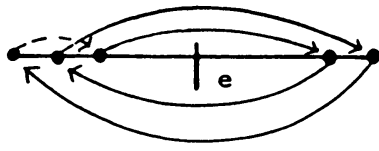

Figure 4.2. A pendulum orbit of period 5 in case $I$.

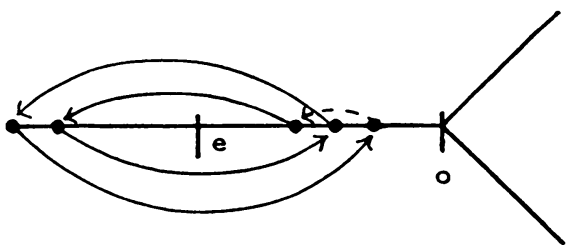

FIgURE 4.3. A pendulum orbit of period 5 in case II.

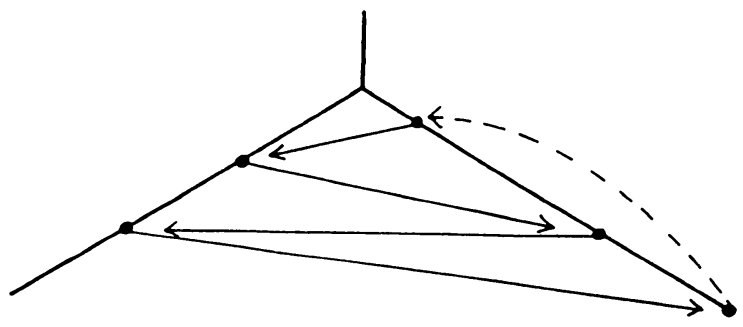

FIGURE 4.4. A pendulum orbit of period 5 in case III. 

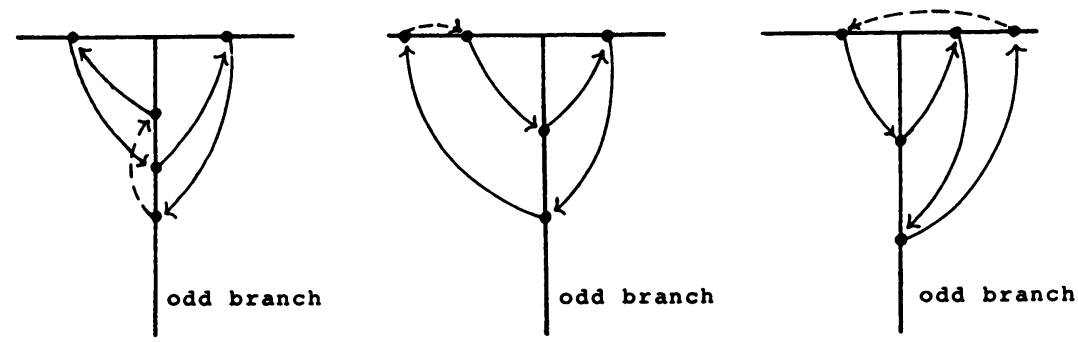

FIGURE 4.5. Several types of pendulum orbits of period 5 in case IV.

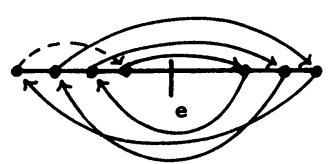

FIGURE 4.6. A pendulum orbit of period 7 in case $I$.

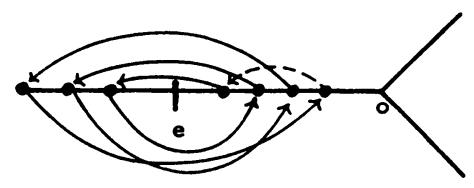

FIGURE 4.7. A pendulum orbit of period 7 in case II.

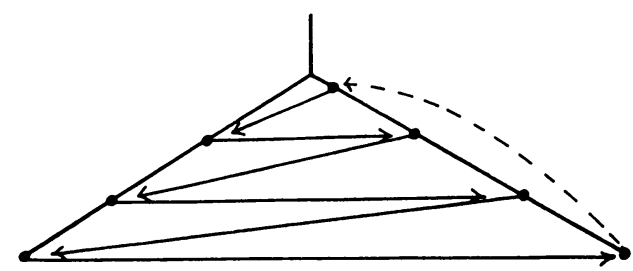

FIGURE 4.8. A pendulum orbit of period 7 in case III.
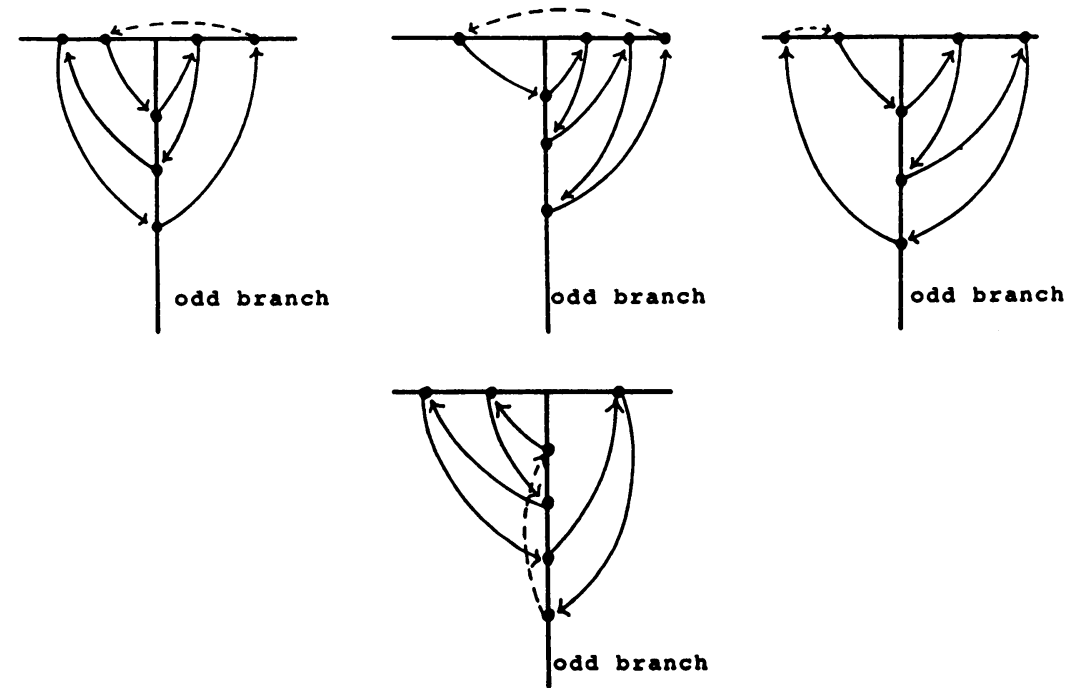

FIGURE 4.9. Several types of pendulum orbits of period 7 in case IV. 

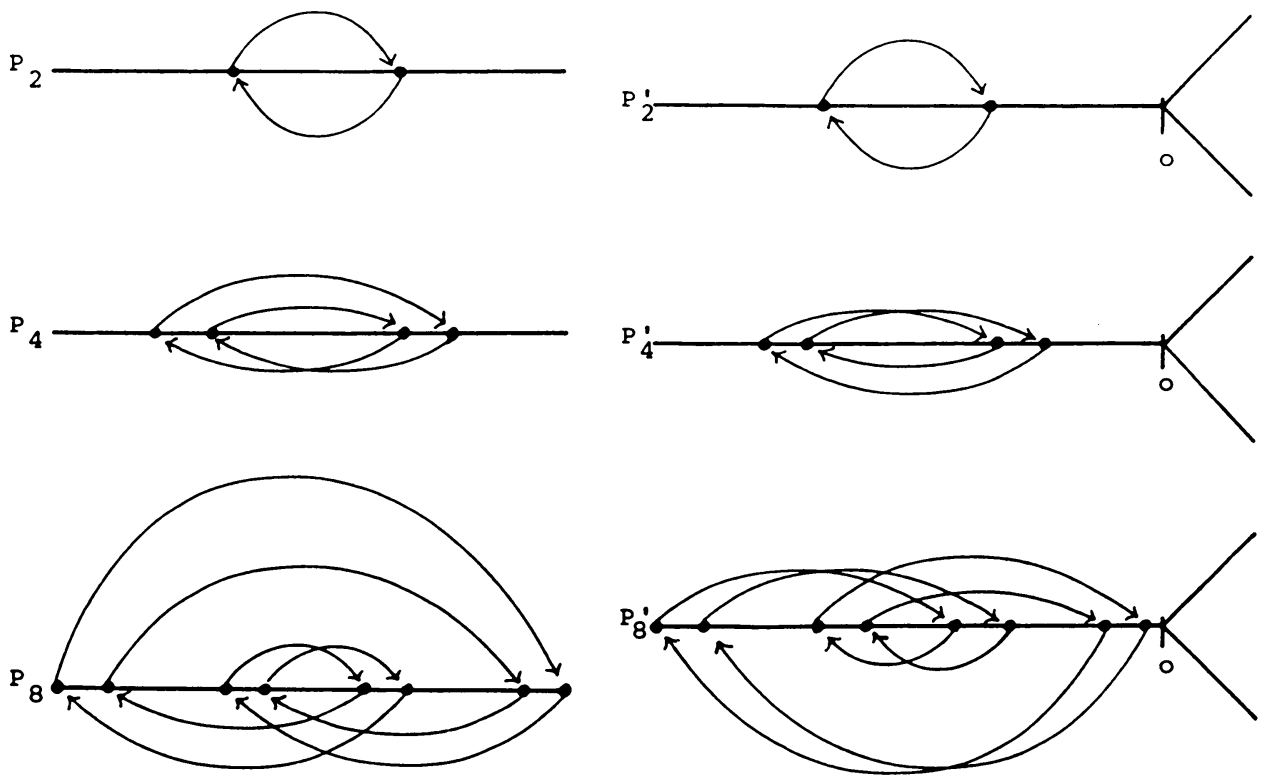

Figure 4.10. Pendulum orbits of periods 2,4 , and 8 in case I, where $P_{4}$ (resp. $P_{8}$ ) is a 2-extension of $P_{2}$ (resp. $P_{4}$ ).

Figure 4.11. Pendulum orbits of periods 2,4 , and 8 in case II, where $P_{4}^{\prime}$ (resp. $P_{8}^{\prime}$ ) is a 2-extension of $P_{2}^{\prime}$ (resp. $P_{4}^{\prime}$ ).

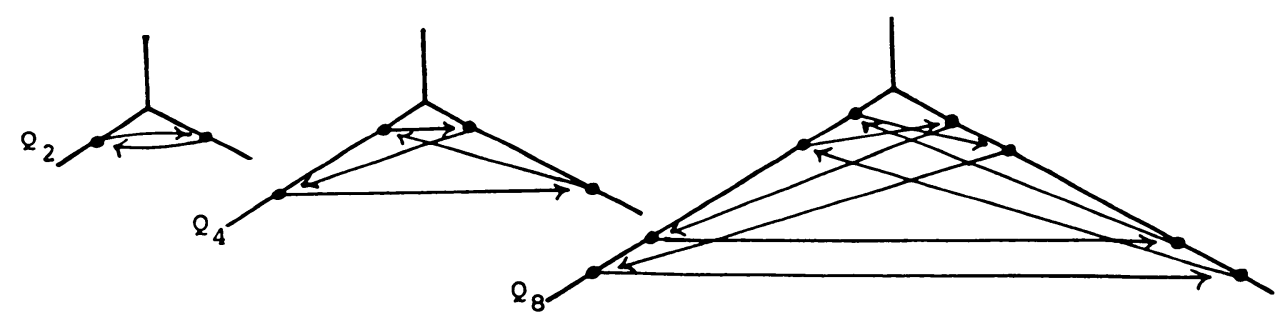

Figure 4.12. Pendulum orbits of periods 2,4 , and 8 in case III, where $Q_{4}$ (resp. $Q_{8}$ ) is a 2-extension of $Q_{2}$ (resp. $Q_{4}$ ).

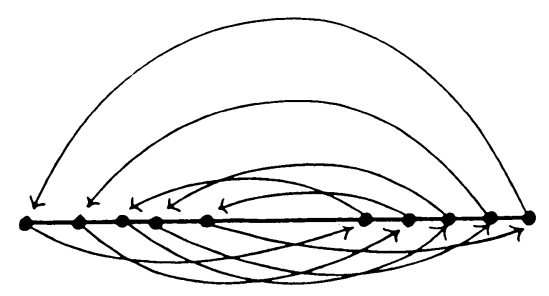

Figure 4.13. A pendulum orbit of period 10 in case $I$. $R$ is the pendulum orbit given in Figure 4.2 and $Q$ is the pendulum orbit of period 2 .

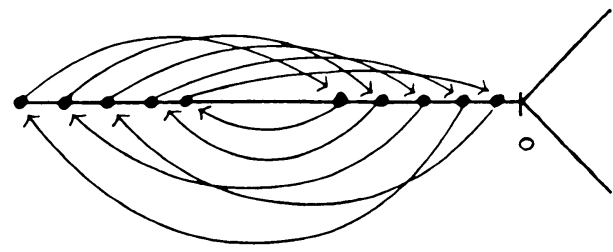

Figure 4.14. A pendulum orbit of period 10 in case $I$. $R$ is the pendulum orbit given in Figure 4.3 and $Q$ is the pendulum orbit of period 2 . 


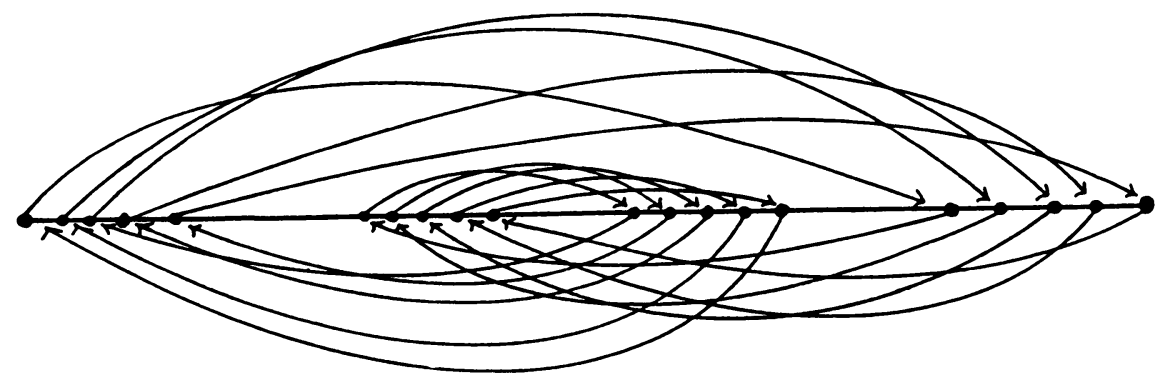

FIGURE 4.15. A pendulum orbit of period 20 in case $\mathrm{I} . R$ is the pendulum orbit given in Figure 4.2 and $Q$ is the pendulum orbit of period 4 given in Figure 4.10.
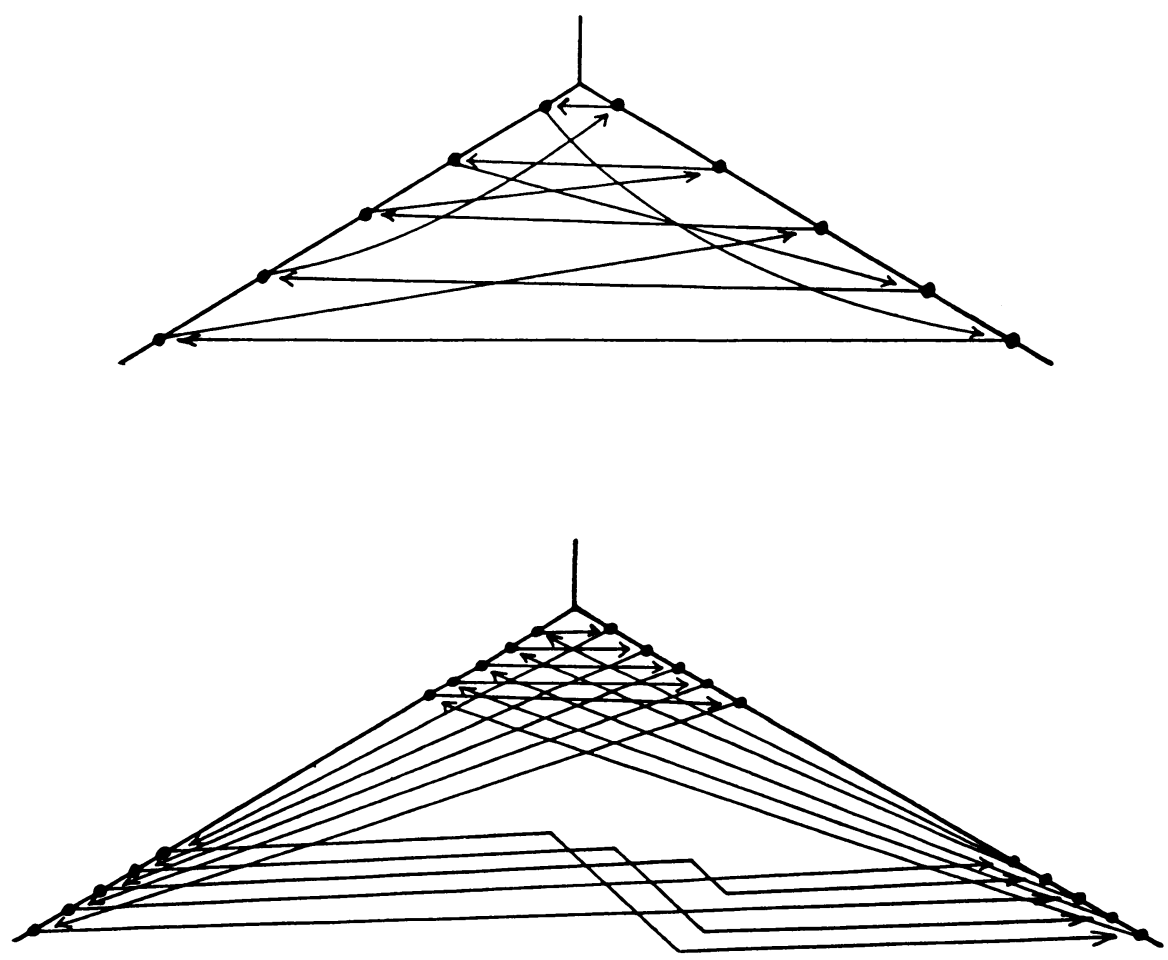

Figure 4.16. Pendulum orbits of periods 10 and 20 in case III. In both cases $R$ is the pendulum orbit of period 5 given in Figure 4.3 (in the interval instead of $Y$ ) and $Q$ is the pendulum orbit of Figure 4.12 of periods 2 and 4 , respectively.

Remark 4.17. It is easy to see that for every $m$ there exist maps $f \in \mathbf{I}$ and $g \in \mathbf{Y}$, each of them having a pendulum orbit of period $m$ (see Remarks 3.2 and 3.5).

Remark 4.18. In the case of maps $f \in \mathbf{I}$, the definition of pendulum orbits coincides with the definition of simple orbits (see [Št, Bl3, C, ALS, H]). 
Now, we consider the directed case. Our assumptions are: $f \in \mathbf{Y}$ and $P$ is a directed periodic orbit of $f$ of period $m$. We assume without loss of generality that the direction of smallest arrows is +1 .

An arrow $A$ will be called black if $\operatorname{dir}(A)=1$, red if $\operatorname{dir}(A)=-1$, and green if $\operatorname{dir}(A)=0$. Red and green arrows will be called colored.

The branch $\mathrm{br}_{j}$ will be called next after $\mathrm{br}_{i}$ if $j \equiv i+1$; it will be called previous if $j \equiv i-1$.

Now, we start to define directed orbits.

Definition 4.19. $P$ will be called a single green (red) orbit if (see Figures 4.20 and 4.21):

(i) $P$ has exactly one colored arrow $A$ and this arrow is green (resp. red).

(ii) If we denote $x_{i}=f^{i}(e(A)), i=0,1,2, \ldots, m-1$, then $x_{i}<x_{i+3}$ for $0 \leq i \leq m-4$.
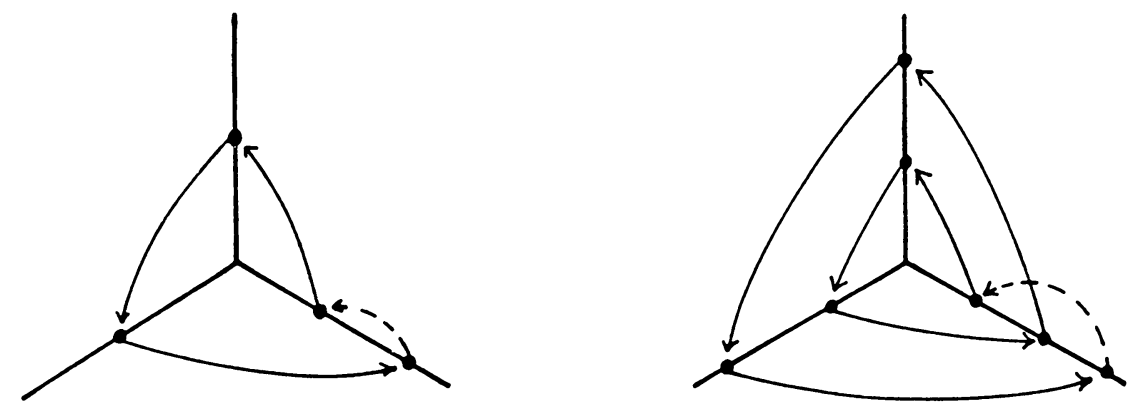

FIGURE 4.20. Single green orbits of periods 4 and 7.
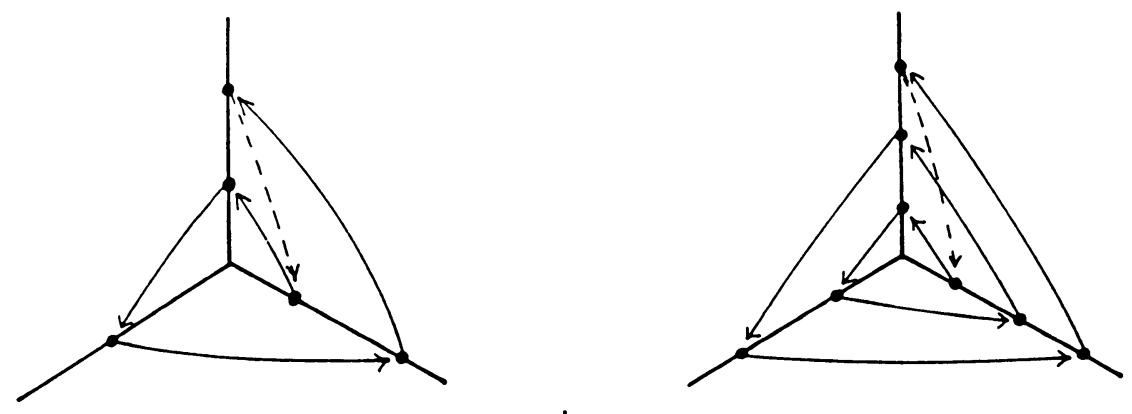

FIGURE 4.21. Single red orbits of periods 5 and 8.

Remark 4.22. It is easy to see that for each $m \geq 4$ if $m \equiv 1$ (resp. $m \equiv 2$ ) there exists a map $f \in \mathbf{Y}$ having a single green (resp. red) orbit of period $m$ and that such orbit is unique up to $E$-equivalence. 
Definition 4.23. $P$ will be called a box green (red) orbit if $m$ is even and $P$ is a 2-extension of a single green (resp. red) orbit of period $m / 2$ (see Figures 4.24 and 4.25 ).
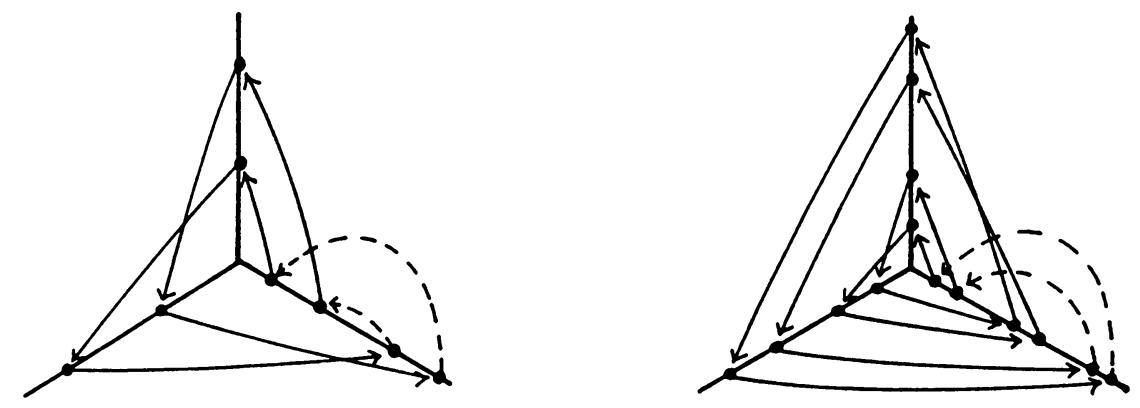

FIGURE 4.24. Box green orbits of periods 8 and 14 obtained by making 2-extensions of the single green orbits shown in Figure 4.20.
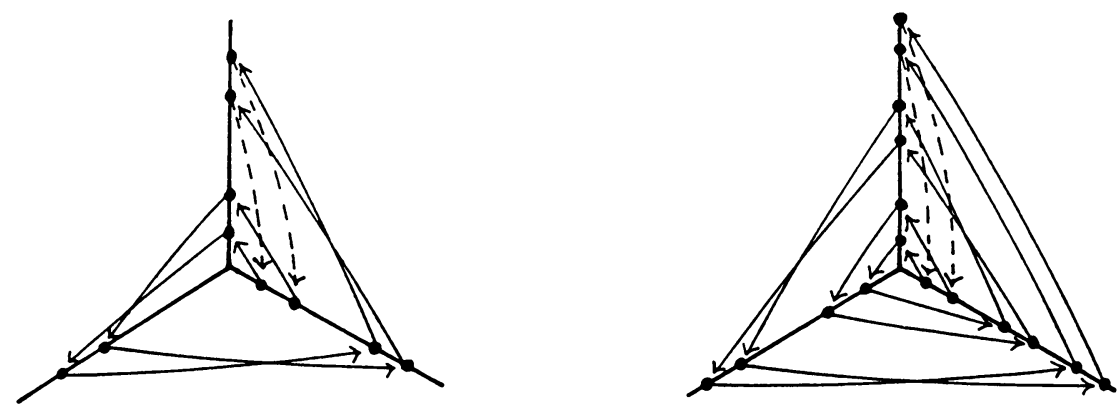

FIgURE 4.25. Box red orbits of periods 10 and 16 obtained by making 2-extensions of the single red orbits shown in Figure 4.21.

Remark 4.26. From Remarks 4.22 and 3.2 it follows that for each $m \geq 8$ if $m \equiv 2$ (resp. $m \equiv 1$ ) and $m$ is even, then there exists a map $f \in \mathbf{Y}$ having a box green (resp. red) orbit of period $m$.

Definition 4.27. $P$ will be called a double green (red) orbit if (see Figures 4.284.31):

(i) $m$ is odd.

(ii) $P$ has exactly two colored arrows, $A$ and $B$, and these arrows are both green (resp. red).

(iii) If we denote by $p$ and $q$ the integers such that $0 \leq p \leq m-2$, $0 \leq q \leq m-2, f^{p}(e(A))=b(B), f^{q}(e(B))=b(A)$, and set $n=$ $(m-5) / 2, x_{i}=f^{i}(e(A))$ for $i=0,1, \ldots, p$ and $y_{i}=f^{i}(e(B))$ for $i=0,1, \ldots, q$, then

(iii.1) $y_{i}<x_{p-n+i}$ for $\max (0, n-p) \leq i \leq \min (n, q)$,

(iii.2) $x_{i}<y_{q-n+i}$ for $\max (0, n-q) \leq i \leq \min (n, p)$,

(iii.3) $x_{i}<x_{i+3}$ for $0 \leq i \leq p-3$,

(iii.4) $y_{i}<y_{i+3}$ for $0 \leq i \leq q-3$. 
Notice that $p+q=m-2=2 n+3$. Observe also that replacing $A, p, x$, $i$ by $B, q, y, j$ and vice versa does not affect the above definition.
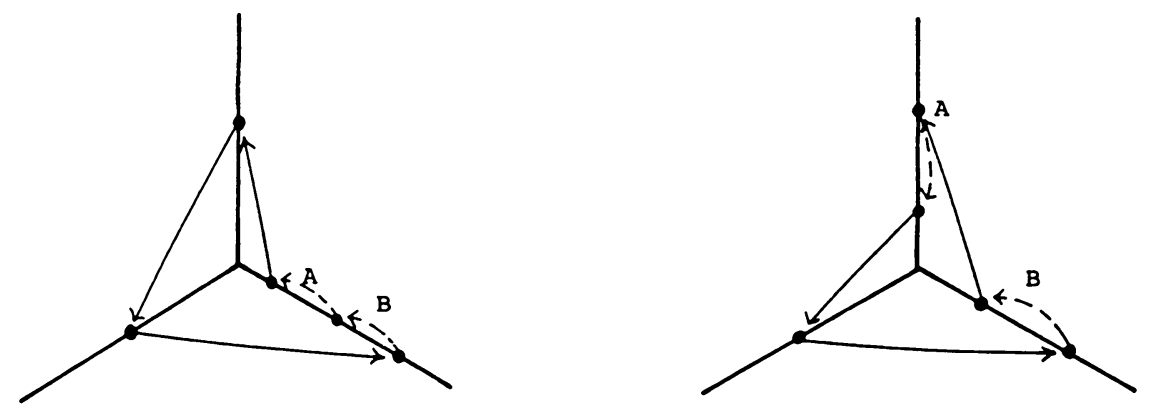

FIGURE 4.28. Double green orbits of period 5, with $q$ equal 0 and 1 , respectively.
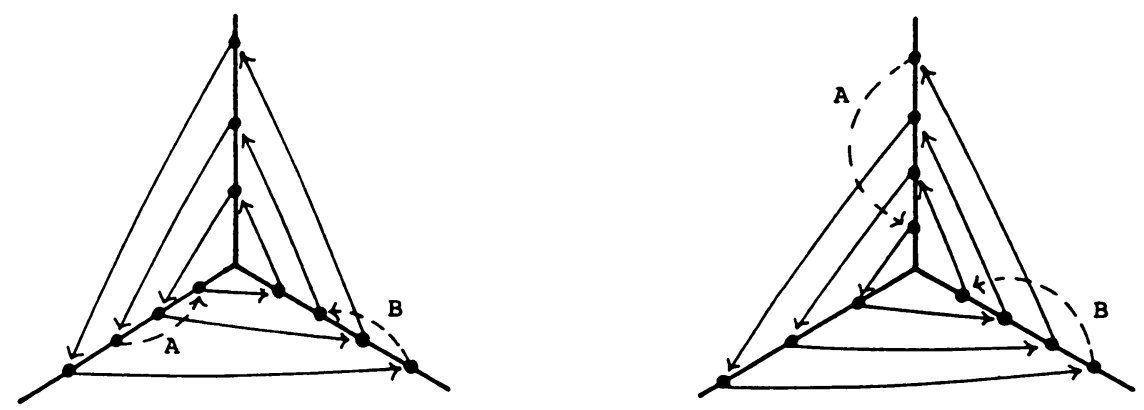

FIGURE 4.29. Double green orbits of period 11 , with $q$ equal 2 and 4 , respectively.
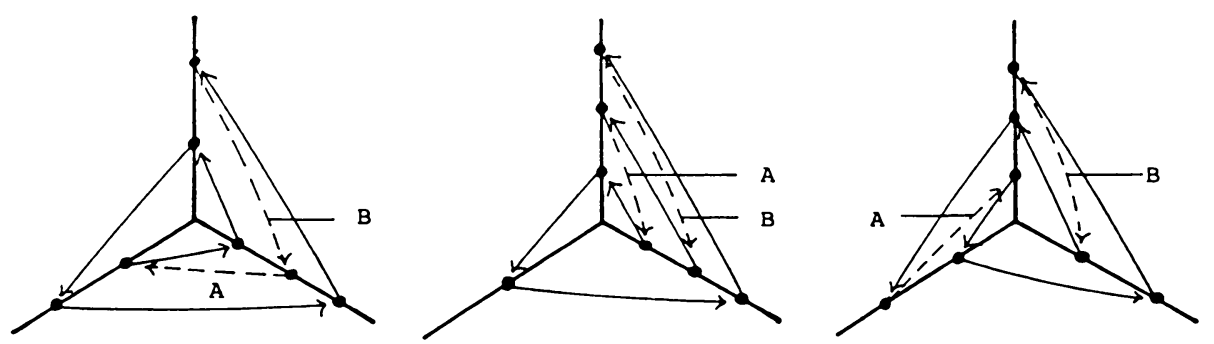

FIGURE 4.30. Double red orbits of period 7, with $q$ equal 0,1 , and 2 , respectively.
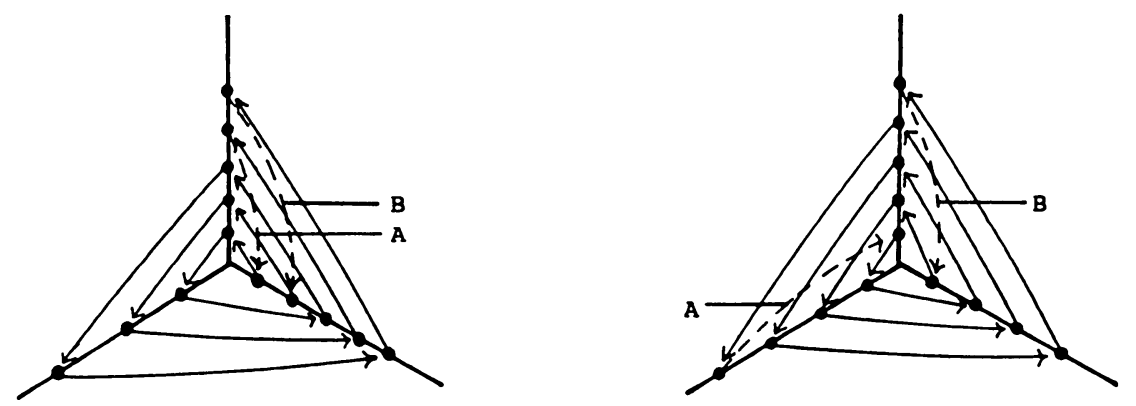

FIGURE 4.31. Double red orbits of period 13, with $q$ equal 4 and 5 , respectively. 
Lemma 4.32. From (iii.1) and (iii.2) of Definition 4.27 it follows that

(a) $x_{i}<x_{i+3}$ for $\max (0, n-q) \leq i \leq \min (n, p-3)$.

(b) $y_{i}<y_{i+3}$ for $\max (0, n-p) \leq i \leq \min (n, q-3)$.

Proof. (a) Let $\max (0, n-q) \leq i \leq \min (n, p-3)$. By Definition 4.27(iii.2) we then have $x_{i}<y_{j}$, where $j=q-n+i$. We have $\max (0, n-p+3)$ $=\max (0, q-n)=\max (0, n-q)+q-n \leq j \leq \min (n, p-3)+q-n=$ $\min (q, p-3+q-n)=\min (n, q)$ (here we use the equality $p+q=2 n+3$ ). By Definition 4.27(iii.1), we get $y_{j}<x_{p-n+j}=x_{i+3}$. Thus, $x_{i}<x_{i+3}$.

We obtain (b) from (a) by replacing $A, p, x, i$ by $B, q, y, j$ and vice versa.

Lemma 4.33. The following statements hold.

(a) For a single or double orbit, if $x_{i} \sim x_{j}$ and $i<j$ then $x_{i}<x_{j}$.

(b) For a double orbit, if $y_{i} \sim y_{j}$ and $i<j$ then $y_{i}<y_{j}$.

Proof. It follows from the fact that all arrows $\left(x_{i}, x_{i+1}\right)$ and $\left(y_{i}, y_{i+1}\right)$ are black and from Definition 4.19(ii) and Definition 4.27(iii.3, iii.4).

Lemma 4.34. For a double orbit let $0 \leq i \leq p$ and $0 \leq j \leq q$. Then the following conditions are equivalent:

(a) $x_{i} \sim y_{j}$.

(b) $i-j \equiv n-q$.

(c) $j-i \equiv n-p$.

Proof. Since $p+q=2 n+3 \equiv 2 n$, (b) and (c) are equivalent. Without loss of generality, we may assume that $p>q$. Then, since $p+q=2 n+3$, we have $p>n$. By Definition 4.27(iii.1), $y_{0}<x_{p-n}$. Hence, $y_{0} \sim x_{p-n}$. Therefore, $x_{i} \sim y_{j}$ is equivalent to $j-0 \equiv i-(p-n)$, i.e., to (c).

Lemma 4.35. For a double orbit let $0 \leq i \leq p, 0 \leq j \leq q$, and $x_{i} \sim y_{j}$. Then:

(a) If $i-j \leq n-q$ then $x_{i}<y_{j}$.

(b) If $j-1 \leq n-p$ then $y_{j}<x_{i}$.

Proof. (a) Assume that $i \geq n-q$. Since $i-j \leq n-q$, we have $i \leq n-(q-j) \leq$ $n$. Hence, by Definition 4.27(iii.2), $x_{i}<y_{q-n+i}$. Since $q-n+i \leq j$ and $y_{q-n+i} \sim x_{i} \sim y_{j}$, by Lemma 4.33, $x_{i}<y_{j}$.

Assume that $i<n-q$. Since $p=2 n+3-q \geq n-q+3$, there exists $k$ such that $n-q<k \leq p$ and $k \equiv i$. Take the smallest such $k$. Since $x_{i} \sim y_{j}$, by Lemma 4.34, we have $n-q+j \equiv i \equiv k$. By the minimality of $k$, we obtain $k \leq n-q+j$. By the part already proved, $x_{k}<y_{j}$. Hence, in view of Lemma 4.33, $x_{i}<y_{j}$.

(b) We obtain it from (a) by replacing $A, p, x, i$ by $B, q, y, j$ and vice versa.

Lemma 4.36. For a single or double orbit, if $w$ and $z$ are beginnings of black arrows and $w<z$, then $f(w)<f(z)$. 
Proof. If $w=x_{i}$ and $z=x_{j}$, from Lemma 4.33(a), it follows that $i<j$. Since $w$ and $z$ are beginnings of black arrows, $f(w)=x_{i+1}$ and $f(z)=x_{j+1}$. Again from Lemma 4.33(a) we obtain $f(w)<f(z)$.

If $w=y_{i}$ and $z=y_{j}$, we obtain $f(w)<f(z)$ as above, by using Lemma 4.33(b).

Assume now that $w=x_{i}$ and $z=y_{j}$. By Lemma 4.34, we have $i-j \equiv n-q$. By Lemma 4.35(b), $j-i>n-p$. Hence $i-j<p-n=2 n+3-q-n=$ $n-q+3$. Therefore, $i-j \leq n-q$. Since $f(w)=x_{i+1}, f(z)=y_{j+1}$, and $(i+1)-(j+1) \leq n-q$, by Lemma 4.35(a), we obtain $f(w)<f(z)$.

If $w=y_{j}$ and $z=x_{i}$, we replace $A, p, x, i$ by $B, q, y, j$ and vice versa and also obtain $f(w)<f(z)$.

Lemma 4.37. For a double orbit let $q<p$. Then $q \leq n+1$. Moreover,

(a) If $q \leq n$ then $x_{n-q}<y_{0}<x_{n-q+3}$.

(b) If $q=n+1$ then $x_{0}<y_{1}<x_{3}$.

Proof. Since $p+q=2 n+3$, we obtain $q<(2 n+3) / 2$ and hence $q \leq n+1$.

Assume that $q \leq n$. By Lemma 4.34, $y_{0} \sim x_{n-q}$ and $y_{0} \sim x_{p-n}=x_{n-q+3}$. By Lemma 4.35, we obtain (a).

If $q=n+1$, then $p=n+2$ and, by Lemma 4.34, $y_{1} \sim x_{0} \sim x_{3}$. By Lemma 4.35, we obtain (b).

Proposition 4.38. Assume that a directed periodic orbit of period $m$ satisfies (i) and (ii) of Definition 4.27. With the notation of Definition 4.27(iii), if $p>q$, then the conditions (iii.1), (iii.2), (iii.3), and (iii.4) of Definition 4.27 are equivalent to the following conditions:

(i) If $w$ and $z$ are beginnings of black arrows and $w<z$, then $f(w)<$ $f(z)$.

(ii) If $q \leq n$, then $x_{n-q}<y_{0}<x_{n-q+3}$, and if $q=n+1$, then $x_{0}<y_{1}<$ $x_{3}$.

Proof. If (iii.1), (iii.2), (iii.3), and (iii.4) of Definition 4.27 are satisfied, then (i) and (ii) follow from Lemmas 4.36 and 4.37.

Assume that (i) and (ii) are satisfied. Notice that $q \leq n+1$ and, hence, (ii) covers all possible cases. Notice that $n-q+3=p-n$ and, if $q=n+1$, then $1=q-n$ and $3=p-n+1$. Hence, we obtain (iii.1), (iii.2), and (iii.3) of Definition 4.27 from (i) and (ii) by induction. Then (iii.4) follows easily.

Remark 4.39. Since in Definition 4.27 we may replace $A, p, x, i$ by $B, q$, $y, j$ and vice versa and the definition will remain the same, we may assume that $p>q$. Hence, Proposition 4.38 gives us another equivalent definition of double orbits. From this new definition it is easy to see that for every $m \geq 5, m$ odd, and for every $q \in\{0,1, \ldots,(m-3) / 2\}$, if $m \equiv 2$ (resp. $m \equiv 1$ ), there exists a map $f \in Y$ having a double green (resp. red) periodic orbit of period $m$, with the prescribed $q$, and such an orbit is unique up to $E$-equivalence.

Now we define the last type of primary orbits. 
Definition 4.40. Assume that $f \in \mathbf{Y}$ and $P$ is a directed periodic orbit of $f$ of period $m$. We say that $P$ is a twist orbit in the following cases (see Figures 4.41 and 4.42 ).

(1) If $m=3.2^{k}, k \geq 0$, we use induction. If $k=0$, then $P$ is twist. If $k \geq 1$ and twist orbits of period $3.2^{k-1}$ are defined, then $P$ is twist if it is a 2-extension of a twist orbit of period $3.2^{k-1}$.

(2) If $m=3.2^{k} . n, n$ is odd, and $n \geq 3$, then $P$ is twist if it is an $R$ extension of a twist orbit of period $3.2^{k}$, where $R$ is a pendulum orbit of period $n$.
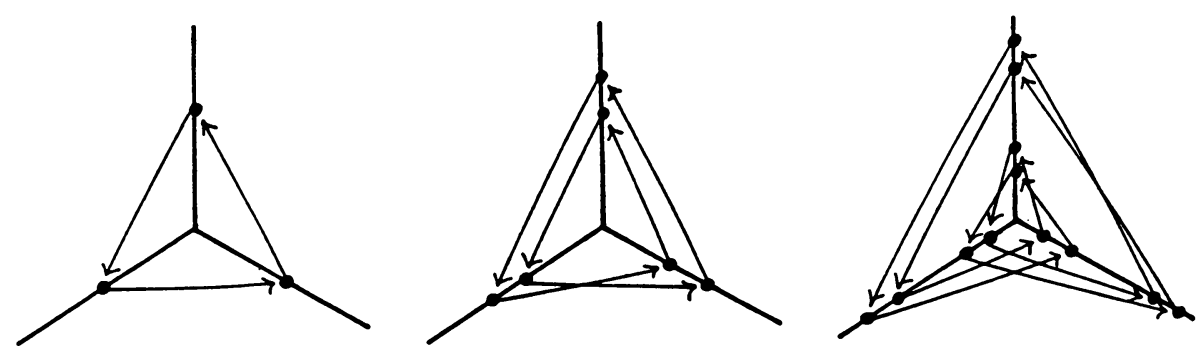

FIGURE 4.41. Twist orbits of period 3,6, and 12. Each orbit with period different from 3 is a 2-extension of the previous one.
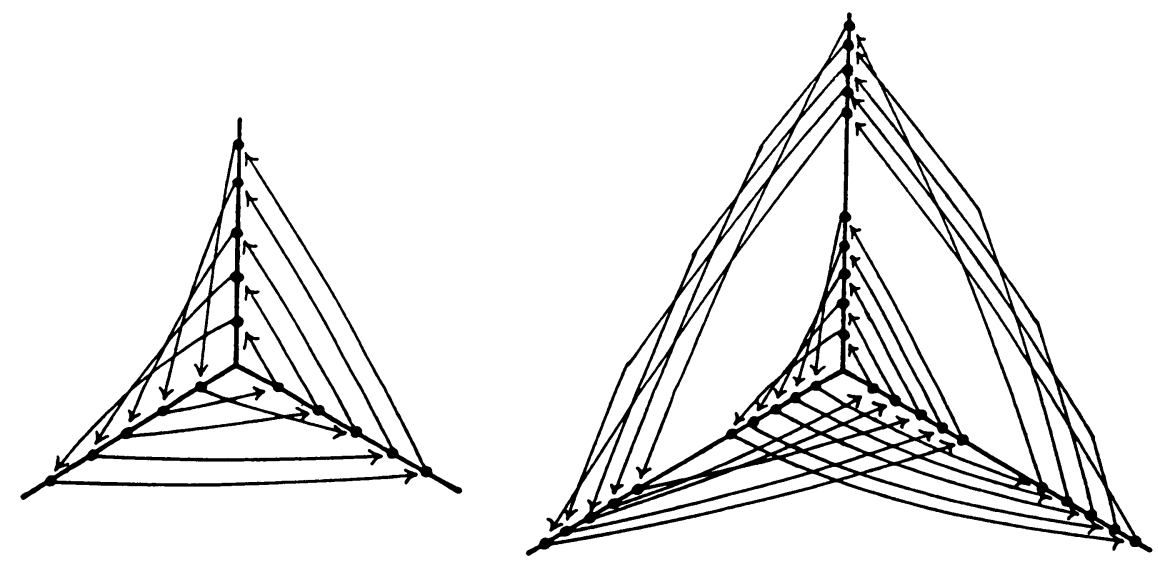

FIgURE 4.42. Twist orbits of periods 15 and 30 . The orbit of period 15 (resp. 30) is an $R$-extension of the twist orbit of period 3 (resp. 6) shown in Figure 4.41, where $R$ is the pendulum orbit of period 5 shown in Figure 4.3.

Remark 4.43. It is easy to see (use Remarks 4.17 and 3.5) that for each $m \equiv 0$ there exists a map $f \in \mathbf{Y}$ having a twist orbit of period $m$.

\section{Proofs OF PRIMARITY}

In this section we prove that the orbits defined in the previous section are primary. We start with the undirected case. Again we consider it in parallel with the case of maps of the interval. Hence the assumptions are that $f \in \mathbf{X}$ 
and $P$ is a pendulum orbit of $f$ of period $m$. We have one of the cases I, II, III, and IV of the previous section (There are no pendulum orbits in case V.)

Remark 5.1. Let $P$ be a periodic orbit of an $E P$-adjusted map $f \in \mathbf{X}$. Since we are only interested in the existence, nonexistence, and behavior of periodic orbits of period larger than one, there is no basic difference between cases I, II, and III. First notice that, since the map $f$ is $E P$-adjusted, we only have to take into account the $E P$-graph of $f$ in $\operatorname{Span}(E P)$. Now the only difference between cases I and II is that in case II, such a graph contains the interval $[0, \min (P)]$. Since $f(0)=0$, in this interval there is no periodic orbit of period larger than one. Hence case II can be reduced to case I. The difference between case I and case III is that, in case III, always $0 \in \operatorname{Span}(P)$ and $f(0)=0$. But in case I, clearly there exists a fixed point $e$ of $f$ such that $e \in \operatorname{Span}(P)$ (if there exist many, choose one). Then we reduce case I to case III by letting $e$ in case I play the role of 0 in case III. If $P$ is pendulum of odd period and we are in case I, this fixed point is given by Definition 4.1. If $P$ is pendulum of even period and we are in case I, from the definition it follows that there exists a fixed point $e \in \operatorname{Span}(P)$ such that $\operatorname{Card}([\min (P), e] \cap P)=\operatorname{Card}([e, \max (P)] \cap P)=$ $\operatorname{Card}(P) / 2$. This point $e$ is the one which has to play the role of 0 to reduce a pendulum orbit in case I to a pendulum orbit in case III. Therefore, from now on we only consider cases III and IV.

Lemma 5.2. Let $P$ be a pendulum orbit of period $m=2^{k}, k \geq 1$, of an EPadjusted map $f \in \mathbf{Y}$. Then $f$ has periodic orbits of periods $1,2,2^{2}, \ldots, 2^{k}$ and no others.

Proof. We use induction. For $k=1$ this is obvious. Assume that we know it for pendulum orbits of period $2^{k-1}$. If $m=2^{k}$ and $P$ is a pendulum orbit of period $m$, then by Definition 4.1, $P$ is a 2-extension of a pendulum orbit $Q$ of period $2^{k-1}$. We may assume that the map $h$ for which $Q$ is a periodic orbit is $E Q$-adjusted. By the induction hypothesis, $h$ has periodic orbits of periods $1,2,2^{2}, \ldots, 2^{k-1}$ and no others. By Lemmas 3.8 and 3.11 and Remark 3.6 it follows that $f$ has periodic orbits of periods $1,2,2^{2}, \ldots, 2^{k}$ and no others.

Lemma 5.3. Let $P$ be an $R$-extension of $Q$. If $Q$ is a pendulum orbit of period $2^{k}$ and $R$ is primary of period larger than one, then $P$ is primary.

Proof. Since the period of $R$ is larger than one, the period $m$ of $P$ is larger than $2^{k}$. We may assume that $Q$ is a periodic orbit of an $E Q$-adjusted map $h$. From Lemma 5.2 it follows that $h$ has no periodic orbits of period $m$. Hence, by Lemma $3.12, P$ is primary.

Proposition 5.4. Pendulum orbits are primary.

Proof. Let $P$ be a pendulum orbit of period $m$ of an $E P$-adjusted map $f$. Assume first that $m$ is odd. Let $x_{i}$ be as in the definition of pendulum orbits of odd period. If a loop of the $E P$-graph of $f$ changes the parity of a branch at each step, then it has even length. Hence, if we have a loop of length $m$ in our 
graph, it has to go through a step preserving parity of a branch. If we denote by $I_{i}$ the basic interval with larger endpoint equal to $x_{i}$ for $i=0,1,2, \ldots, m-$ 1 , then this is the step from $I_{m-1}$ to $I_{0}$ (this follows from the definition of pendulum orbit). We claim that if $I_{i} f$-covers $I_{j}$, then $j \leq i+1$. If $i=m-1$, this is obvious. Assume that $i<m-1$. Then $f\left(\operatorname{Span}\left\{x_{0}, x_{1}, \ldots, x_{i}\right\}\right)=$ $\operatorname{Span}\left(\left\{x_{0}, x_{1}, \ldots, x_{i+1}\right\}\right)$. Consequently, if $I_{i} f$-covers $I_{j}$, then by (ii) of the definition of pendulum orbit, $j \leq i+1$. This proves the claim. Therefore, our loop is $I_{0} \rightarrow I_{1} \rightarrow I_{2} \rightarrow \cdots \rightarrow I_{m-1} \rightarrow I_{0}$. Hence, from Proposition 1.11 , every periodic orbit of $f$ of period $m$ is associated to the above loop. From statements (a.3) and (b) of Proposition 1.10 it follows that $P$ is the only periodic orbit of $f$ of period $m$. By the First Theorem (2.3), $P$ is primary. This ends the proof for $m$ odd.

Now let $m=2^{k}, k \geq 1$. Clearly, if $k=1$, then $P$ is primary. If $k>1$, then by Definition 4.1 and Lemma 5.3, $P$ is also primary (here we also use Remark 3.6).

If $m=2^{k} . n$ with $n \geq 3, n$ odd, then by Definition 4.1 , Lemma 5.3, and the part of the proposition already proved, $P$ is also primary.

Now we are going to consider the directed case.

Lemma 5.5. Let $P$ be a twist orbit of period $m=3.2^{k}$ with $k \geq 0$ of an $E P$. adjusted map $f \in \mathbf{Y}$. Then $f$ has periodic orbits of period $3,3.2,3.2^{2}, \ldots, 3.2^{k}$ and no others.

Proof. We use induction. For $k=0$ it is obvious. Assume that we know this for twist orbits of period $3.2^{k-1}$. If $m=3.2^{k}$ and $P$ is a twist orbit of period $n$, then by Definition $4.40 P$ is a 2-extension of a twist orbit $Q$ of period $3.2^{k-1}$. We may assume that the map $h$ for which $Q$ is a periodic orbit is $E Q$-adjusted. By the induction hypothesis, $h$ has periodic orbits of periods $3,3.2,3.2^{2}, \ldots, 3.2^{k-1}$ and no others. By Lemmas 3.8 and 3.11 and by Remark 3.6, $f$ has periodic orbits of periods $3,3.2,3.2^{2}, \ldots, 3.2^{k}$ and no others.

Lemma 5.6. Let $P$ be an $R$-extension of $Q$. If $Q$ is a twist orbit of period $3.2^{k}$ and $R$ is primary of period larger than one, then $P$ is primary.

Proof. Since the period of $R$ is larger than one, the period $m$ of $P$ is larger than $3.2^{k}$. We may assume that $Q$ is a periodic orbit of an $E Q$-adjusted map $h$. From Lemma 5.5 it follows that $h$ has no periodic orbits of period $m$. Hence, by Lemma 3.12, $P$ is primary.

Proposition 5.7. Twist orbits are primary.

Proof. Let $P$ be a twist orbit of period $m$ and let $f$ be $E P$-adjusted. If $m=3$, then $P$ is obviously primary (use the First Theorem (2.3)).

If $m=3.2^{k}, P$ is primary by Definition 4.40 and Lemma 5.6 (recall that every periodic orbit of period two is primary). 
If $m=3.2^{k} . n, n \geq 3, n$ odd, then $P$ is primary by Definition 4.40 , Lemma 5.6, and Proposition 5.4.

Now we are going to prove that single, box, and double colored orbits are primary. First, we consider the cases of single and double colored orbits. In both cases we assume that $f$ has an orbit $P$ of period $m$, this orbit is single colored or double colored, and $f$ is $E P$-adjusted. We shall investigate loops of length $m$ in the $E P$-graph of $f$. We take such a loop $\alpha$. We use the notation of Definitions 4.19 and 4.27. In what follows, we start by making several simple observations.

Observation 5.8. In the green (red) case, when going along the loop $\alpha$, we cannot move to the previous branch (resp. stay at the same branch).

Observation 5.9. Let $v$ be the number of times that we do not move to the next branch. Then in the green (red) case we have $m-v \equiv 0$ (resp. $(m-v)-v \equiv 0)$. Since $m \equiv 2$ in the single red and double green cases, and $m \equiv 1$ in the single green and double red cases, we obtain $v \equiv 1$ in the single case and $v \equiv 2$ in the double case.

Observation 5.10. When moving along the loop $\alpha$ we go from a basic interval $I$ to a basic interval not in the next branch; then there are two possibilities. The first is that one of the endpoints of $I$ is the beginning of a black arrow and the other is the beginning of a colored arrow. We call this arrow $C$. In this case we move from $I$ to some basic interval contained in $[0, e(C)]$. The second possibility is that both endpoints of $I$ are beginnings of colored arrows. Then $P$ is a double orbit and we move from $I=[b(A), b(B)]$ to some basic interval contained in $[e(A), e(B)]$.

In the rest of the section we shall distinguish two cases:

Case A. No basic interval has both endpoints equal to beginnings of colored arrows.

Case $\mathrm{B}$. There is a basic interval with both endpoints equal to beginnings of colored arrows.

Notice that the single case always satisfies Case A. To deal with Case B we have to prove some auxiliary lemmas. However, we have to fix our notation first.

Notation. In Case $\mathrm{B}$, we assume that $b(A)<b(B)$. We may make such an assumption without loss of generality because in Case $\mathrm{B}, b(A) \sim b(B)$. We should remember that $b(A)=y_{q}, b(B)=x_{p}, n=(m-5) / 2$, and $p+q=$ $2 n+3$.

Lemma 5.11. In Case $\mathrm{B}, q=n$ and $p=n+3$.

Proof. First, notice that $p \equiv q$. Assume that $q>n$. Then $p<n+3$. Since $p \equiv q$, we have $q \geq n+3$ and $p \leq n$. Hence, $\max (0, n-q) \leq p \leq \min (n, p)$, 
because $p=2 n+3-q>n-q$. Therefore, by Definition 4.27, we have $x_{p}<y_{q-n+p}=y_{n+3} \leq y_{q}$, a contradiction. Hence $q \leq n$.

Assume that $q<n$. Then $p>n+3$. Since $q=2 n+3-p>n-p$ and $q<n$, we have $\max (0, n-p) \leq q \leq \min (n, q)$. By Definition 4.27, we get $y_{q}<x_{p-n+q}=x_{n+3}<x_{p}$, which contradicts our assumption that $y_{q}$ and $x_{p}$ are endpoints of a basic interval. Hence $q \geq n$.

Lemma 5.12. In Case $\mathrm{B}, b(B)$ is the largest point of $P$ on its branch.

Proof. If $x_{i} \sim x_{p}$ for some $i$, then $i \equiv p$ and, by Definition 4.27, $x_{i} \leq x_{p}$. If $y_{i} \sim x_{p}$ for some $i$, then $y_{i} \sim y_{q}$ and $i \equiv q$. By Definition 4.27, $y_{i}<y_{q}$ and, since by Lemma 5.11 and Definition $4.27 y_{q}<x_{p}$, we obtain $y_{i}<x_{p}$. Hence, the point $x_{p}=b(B)$ is the largest point of $P$ on its branch.

Lemma 5.13. In Case $\mathrm{B}$ the points $e(A)$ and $e(B)$ are the endpoints of some basic interval. Moreover, $e(A)<e(B)$.

Proof. Recall that $e(A)=x_{0}$ and $e(B)=y_{0}$. By Lemma 5.11 and Definition 4.27, we have $x_{0}<y_{0}<x_{3}$. Hence, again by Definition 4.27, there are no points of $P$ between $x_{0}$ and $y_{0}$.

In Case $\mathrm{A}$, we call the situation described in Observation 5.10 as the first possibility moving along $C$. In Case $\mathrm{B}$, this applies to $C=A$. By moving along $B$ we shall mean a step from $[b(A), b(B)]$ to $[e(A), e(B)]$ (by Lemma 5.13 , this interval is basic).

Now we go along the loop $\alpha$, perhaps many times. We call the consecutive colored arrows along which we are moving $C_{0}, C_{1}, C_{2}, \ldots$. We consider several possible cases.

Lemma 5.14. Assume that $P$ is a double orbit and $C_{i}=C_{i+1}$ for some $i$. Then between moving along $C_{i}$ and $C_{i+1}$ we make at least $n+3$ steps (where $n$ is from Definition 4.27).

Proof. In Case A we may assume that $C_{i}=C_{i+1}=A$. In Case B we may not make this assumption only in the following situation: we use in the proof the definition of moving along $A$, and its consequences are false if we replace $A, p, x, i$ by $B, q, y, j$ and vice versa. However, we have to use the definition of moving along $A$ only in two places: to conclude that after moving along $A$ we are in one of the basic intervals contained in $\left[0, x_{0}\right]$, and to conclude that to move again along $A$ we need to be in an interval containing $b(A)$. But, in Case B, if we replace $A, p, x, i$ by $B, q, y, j$ and vice versa, both consequences stay true. The first one follows from the fact that, by Lemma $5.13, e(A)<e(B)=y_{0}$. The second one is obvious. Hence, the situation described in the beginning of this paragraph never arises, and therefore even in Case B we may assume that $C_{i}=C_{i+1}=A$.

The above explanations may become clearer after reading the rest of the proof.

After moving along $C_{i}$ we are in one of the basic intervals contained in $\left[0, x_{0}\right]$. Since during the next $k$ steps which we do before moving along $C_{i+1}$, 
we move to the next branch each time, by Lemma 4.36, we are in the intervals $\left[0, x_{1}\right],\left[0, x_{2}\right], \ldots,\left[0, x_{\min (p, k)}\right]$ after $1,2, \ldots, \min (p, k)$ steps, respectively. Two cases are possible:

1. $p \geq k$. Since in the next step we move along $A$ and $\min (p, k)=k$ we have $b(A) \in\left[0, x_{k}\right]$. Hence $b(A) \leq x_{k}$. We have three subcases.

(1.i) $\max (0, n-q) \leq k \leq \min (n, p)$. Then, by Definition 4.27, $x_{k}<y_{q-n+k}$. But since $x_{k} \geq b(A)=y_{q}$, we get $y_{q}<y_{q-n+k}$ and, again by Definition 4.27. $q<q-n+k$. Hence $k>n$.

(1.ii) $k<\max (0, n-q)$. Then we have $0<k<n-q$ and we replace $k$ by $k^{\prime}=n-q, n-q+1$, or $n-q+2$ such that $k^{\prime} \equiv n$. Since $n-q \leq n$ and $k^{\prime} \equiv n$, we have $k^{\prime} \leq n$. Also we have $n-q+2<p$, because $p+q=2 n+3$. Hence, $k^{\prime} \leq \min (n, p)$. On the other hand, $q+p-n=n+3 \equiv n \equiv k^{\prime}$. Hence, $q-k^{\prime} \equiv n-p$ and, by Lemma 4.34 , we have $y_{q} \sim x_{k^{\prime}}$. Therefore $y_{q}=b(A) \leq x_{k}<x_{k^{\prime}}$ because $k^{\prime}>k$ and by Definition 4.27(iii.3). Thus, we can proceed as in (1.i). Then we obtain $k^{\prime}>n$. This is a contradiction.

(1.iii) $k>\min (n, p)$. Since $k \leq p$, we have $k>n$. Hence in case 1 we have $x>n$.

2. $p<k$. Suppose that $k \leq n$. Then $p<n$ and, hence, $q>n+3>n$ (because $p+q=2 n+3$ ). Therefore, by Definition 4.27(iii.1, iii.2) we have $y_{n}<x_{p}<y_{n+3}$. Moreover, if $p \geq 3$, then $x_{p-3}<y_{n}$. Hence the largest point $z$ of $P$ such that $z<x_{p}$ is equal to $y_{n}$. We continue moving along the loop $\alpha$ starting from the $(p+1)$ th step. At each step we move to the next branch and we are consecutively in $\left[0, y_{n+1}\right],\left[0, y_{n+2}\right], \ldots,\left[0, y_{n+k-p}\right]$. Since in the next step we have to move along $A$, we have $b(A) \in\left[0, y_{n+k-p}\right]$. Hence $b(A) \leq y_{n+k-p}$. Since $b(A)=y_{q}$, by Definition 4.27, we get $q \leq n+k-p$ and, consequently, $n+3 \leq k$, a contradiction. Hence, in case 2 we also have $k>n$.

Clearly, in the green case we have $k \equiv 0$ and in the red case $k \equiv 1$. We note that, by Definition 4.27 , in the green case we have $m \equiv 2$ and $n \equiv 0$, and in the red case $m \equiv 1$ and $n \equiv 1$. Hence, $k \equiv n$. Since $k>n$ we obtain $k \geq n+3$.

We denote by $I_{i}$ the basic interval with larger endpoint equal to $x_{i}$, and by $J_{j}$ the interval with larger endpoint equal to $y_{j}$. Also, in the single case we set $p=m-1$.

Lemma 5.15. Assume that either $P$ is a double orbit and $C_{i}=A, C_{i+1}=B$ for some $i$, or $P$ is a single orbit. Then between moving along $C_{i}$ and $C_{i+1}$ we make at least $p$ steps (where $p$ is from Definition 4.27 in the double case and $p=m-1$ in the single case). Moreover, if we make exactly $p$ steps, then we move through the intervals $I_{0}, I_{1}, I_{2}, \ldots, I_{p}$.

Proof. We move through basic intervals contained in $\left[0, x_{0}\right],\left[0, x_{1}\right], \ldots$, $\left[0, x_{p}\right]$ consecutively. Hence, by Lemma 4.36, we cannot reach $b\left(C_{i+1}\right)=x_{p}$ in less than $p$ steps. Since the only point of $\left[0, x_{j}\right]$ which is mapped to $x_{j+1}$ 
is $x_{j}$ for $j=0,1, \ldots, p-1$ (we recall that $f$ is $E P$-adjusted), if we make exactly $p$ steps, we have to use the intervals $I_{0}, I_{1}, \ldots, I_{p-1}$ consecutively. The next step is along $C_{i+1}$. Hence, the corresponding basic interval has $b\left(C_{i+1}\right)$ as endpoint. But since it is contained in $\left[0, x_{p}\right]=\left[0, b\left(C_{i+1}\right)\right]$, it has to be $I_{p}$.

Lemma 5.16. Let $P$ be a double orbit, $C_{i}=B$, and $C_{i+1}=A$ for some $i$. Then between moving along $C_{i}$ and $C_{i+1}$ we make at least $q$ steps (where $q$ is from Definition 4.27). Moreover, if we make exactly $q$ steps, then we move through the intervals $J_{0}, J_{1}, \ldots, J_{q}$.

Proof. In Lemma 5.15, we may replace $A, p, x, i$ by $B, q, y, j$ and vice versa. In case $A$ it is obvious. In case $B$ we can do that by the reasons given at the beginning of the proof of Lemma 5.14.

Proposition 5.17. Single and double colored orbits are primary.

Proof. Let $P$ be a periodic orbit of a map $f \in \mathbf{Y}$ of period $m$. We may assume that $f$ is $E P$-adjusted.

Assume first that $P$ is a single colored orbit. From Observation 5.9 it follows that when going along a loop of the $E P$-graph of $f$ of length $m$ we have to move along the colored arrow. By Lemma 5.15, the loop associated to $P$ is the only loop of length $m$ in the EP-graph of $f$. From Proposition 1.11 and statements (a.3) and (b) of Proposition 1.10, it follows that $P$ is the only periodic orbit of $f$ of period $m$. By the First Theorem (2.3), $P$ is primary.

Now assume that $P$ is a double colored orbit. From Observation 5.9 it follows that when going along a loop of the $E P$-graph of $f$ of length $m$ we have to move along a colored arrow at least twice. If we move twice in a row along the same colored arrow, then in the whole loop we either use only one arrow to move along it or we use both arrows. In the first case, by Lemma 5.14, $m \geq 2(n+3)+2=m+3$, a contradiction. In the second case, by Lemmas 5.14 , 5.15, and 5.16, $m \geq p+q+n+3+3=m+n+4$, again a contradiction. Hence, we do not move along the same colored arrow twice in a row. By Lemmas 5.15 and 5.16, we get that the loop associated to $P$ is the only loop of length $m$ of the EP-graph of $f$. As above, by Proposition 1.11, statements (a.3) and (b) of Proposition 1.10, and the First Theorem (2.3), it follows that $P$ is primary.

Proposition 5.18. Box colored orbits are primary.

Proof. Since a periodic orbit of period two is primary, in view of Lemma 3.12 and Remark 3.6, it suffices to prove that if $Q$ is a single colored orbit of an $E Q$-adjusted map $h$ of period $l$, then $h$ has no periodic orbits of period $2 l$. If $\alpha$ is a loop in the $E Q$-graph of $h$, associated to a periodic orbit of period $2 l$, it has to go along the colored arrow at least twice. To see this, denote by $v$ the number of times that we move along a colored arrow, as in Observation 5.9. We have $2 l-v \equiv 0$ in the green case and $2 l-2 v \equiv 0$ in the red case. From Remark 4.22, we have $l \equiv 1$ (resp. $l \equiv 2$ ) in the green (resp. red) case. Hence $v \equiv 2$. 
Since $\alpha$ goes twice along the colored arrow, by Lemma 5.15, $\alpha$ is a repetition two times of the loop associated to $Q$. Hence, $Q$ is associated to $\alpha$. By Proposition 1.10, $\alpha$ does not give a periodic orbit of period $2^{l}$, a contradiction.

\section{GENERAL RULE FOR PRIMARY ORBITS}

Lemma 6.1 (General Rule). If $P$ is a primary periodic orbit of period $m>1$ of $f \in \mathbf{Y}$ such that the points of $P$ lie on at least two branches, then there is no point $x \in P$ such that $f(x)>x$.

Proof. Assume that there exists $x \in P$ such that $f(x)>x$ (notice that this implies $m \geq 3$ ). We may assume that $x$ is the largest point on its branch with this property. Then $x$ is the smaller endpoint of some basic interval $I=[x, y]$ and $I f$-covers itself. Since we only take into account the behavior of $f$ on $E P$, we may assume that $f$ is $E P$-adjusted.

We set $V_{i}=f^{i}(I), i=0,1,2, \ldots$. Since $V_{0} \subset V_{1}$, we have $V_{0} \subset V_{1} \subset$ $V_{2} \cdots$. Since $f$ is $E P$-adjusted, we have

$$
V_{i} \supset \operatorname{Span}\left(\left\{f^{j}(x): j=0,1,2, \ldots, i\right\} \cup\left\{f^{j}(y): j=0,1, \ldots, i\right\}\right) .
$$

Therefore, for some $r, V_{0} \subsetneq V_{1} \subsetneq V_{2} \subsetneq \cdots \subsetneq V_{r}=\operatorname{Span}(E P)$ (since the points of $P$ lie on at least two branches, $\operatorname{Span}(P)=\operatorname{Span}(E P))$. Since

$$
\operatorname{Card}\left(P \cap V_{i+1}\right)>\operatorname{Card}\left(P \cap V_{i}\right) \quad \text { if } i<r
$$

and $\operatorname{Card}\left(P \cap V_{0}\right)=2$, we obtain $r \leq m-2$.

Let $k$ be the smallest integer such that $V_{k}$ contains a basic interval $J \neq I$ which $f$-covers $I$. Since the interval $[0, x] f$-covers $I$, such $k$ exists, and if $0 \in V_{i}$ then $k \leq i$. Therefore, we have

$$
\stackrel{1 \text { step }}{I} \underset{1 \text { step }}{\stackrel{\text { steps }}{\rightleftarrows}} J \text {. }
$$

By Lemma 1.12, there is a loop $I \rightarrow \cdots \rightarrow J \rightarrow I$ in the $E P$-graph of $f$ of length $k+1$. Adding to this loop the loop $\tilde{I} l$ times, we get a loop of length $k+1+l$ which goes through $I l+1$ times. If $l \geq 2$, since $I$ contains only two points of $P$, this loop cannot be associated to $P$. By construction it is nonrepetitive and, by Lemma 1.3, there is a periodic orbit of period $k+1+l$ associated to it. Hence, by the First Theorem (2.3), we cannot have $m-(k+1) \geq$ 2 . Thus, $k>m-3$. Since $k \leq r \leq m-2$, we obtain $k=r=m-2$. Since $r=m-2, \operatorname{Card}\left(P \cap V_{i+1}\right)=\operatorname{Card}\left(P \cap V_{i}\right)+1$ for $i=0,1,2, \ldots, r-1$. Since $k=r$, we have $0 \notin V_{r-1}$.

Now we claim that there is a point $z \in P$ such that $f^{m-1}(z)$ is the only point of $P$ lying on a branch different from $\mathrm{br}_{\text {ind }(x)}$, and the ordering of the points $z_{i}=f^{i}(z), i=1,2, \ldots, m-2$, is either

(i) $z_{m-2}<z_{m-4}<\cdots<z<\cdots<z_{m-5}<z_{m-3}$ or 
(ii) $z_{m-3}<z_{m-5}<\cdots<z<\cdots<z_{m-4}<z_{m-2}$.

In the case $m=3$, since $P$ has points in at least two branches, the claim holds for $z=x$ and (ii) is satisfied. If $m \geq 4$, since $0 \notin V_{1}$, we have $\operatorname{ind}(f(y))=\operatorname{ind}(x)$. Since $\operatorname{Card}\left(V_{0} \cap P\right)=2$ and $\operatorname{Card}\left(V_{1} \cap P\right)=3$, we have either $f(x)=y, f(y)<x$, and $(f(y), x) \cap P=\varnothing$, or $f(y)=x, f(x)>y$, and $(f(x), y) \cap P=\varnothing$.

We prove the claim in the first case. In the other case it follows similarly. We set $z=x$. If $m=4$, then the claim holds and (i) is satisfied. If $m \geq 5$, $V_{1}=[f(y), y]=\left[z_{2}, z_{1}\right]$. Since $\operatorname{Card}\left(V_{2} \cap P\right)=\operatorname{Card}\left(V_{1} \cap P\right)+1, z \in V_{1}$, and $0 \notin V_{2}$, we get that $z_{3}>z_{1}$ and $\left(z_{3}, z_{1}\right) \cap P=\varnothing$. Iterating this process, we have either (i) or (ii). Hence the claim holds because $P$ has points at least in two branches.

Case (i) is impossible, since then $\left[z_{m-2}, z_{m-4}\right] f$-covers $I$, in contradiction to the minimality of $k$ (notice that $\left[z_{m-2}, z_{m-4}\right] \subset V_{m-3}$ ).

In case (ii) we set $K=\left[0, z_{m-3}\right]$ and $L=\left[z_{m-4}, z_{m-2}\right]$. We have $\tilde{K} \rightleftarrows L$. Then we have the loop $K \rightarrow K \rightarrow \cdots \rightarrow K \rightarrow L \rightarrow K$ of length $m$ in the $E P$ graph of $f$. This loop is nonrepetitive and, by Lemma 1.3, there is a periodic orbit of period $m$ associated to this loop and contained in one branch. By the First Theorem, this is a contradiction.

\section{UNDIRECTED THEOREM (FIRST PART)}

Now we start proving the following result.

Theorem 7.1 (Undirected Theorem). Let $P$ be a periodic orbit of $f \in \mathbf{X}$. If $P$ is undirected and primary, then $P$ is pendulum.

In this section we only state and prove some preliminary results. The Undirected Theorem will be proved in $\S 9$.

In the rest of this section we shall assume that $P$ is a periodic orbit of an $E P$-adjusted map $f \in \mathbf{X}$ of period $m$, and $P$ is undirected. We shall also use the notation of $\S 4$ for undirected orbits. We consider here only cases I, II, III, and IV (see §4).

By Remark 5.1, it is enough to consider only case III or IV. Indeed, in the reduction of cases I and II to case III we did not use the assumption that $P$ is pendulum. Here any fixed point $e \in \operatorname{Span}(P)$ can play the role of 0 in the reduction. Recall that cases III and IV are:

(III) $f \in \mathbf{Y}, P$ lies on two branches.

(IV) $f \in \mathbf{Y}, P$ lies on all three branches and all smallest arrows have direction different from 0 .

Hence, in the rest of the section, $m \geq 2$.

Lemma 7.2. Assume that $P$ is pendulum, $m \geq 3$, and $m$ is odd. Then, for every $n$ such that $n>_{s} m, f$ has a periodic orbit $Q$ of period $n$ with $\operatorname{Span}(Q) \subsetneq$ $\operatorname{Span}(P)$. 
Proof. If $n=1$, then Lemma 7.2 follows from Definition 4.1. Now we assume that $n$ is even. There is $i \leq m-1$ such that $x_{i} \sim x_{i+2}$ but $f\left(x_{i}\right)$ and $f\left(x_{i+2}\right)$ lie on different branches (we use the notation of Definition 4.1). Denote $K=\left[0, x_{i}\right], L=\left[x_{i}, x_{i+2}\right]$, and $M=\left[0, x_{i+1}\right]$. We have $K \rightleftarrows M \rightleftarrows L$, which gives us a periodic orbit $Q$ of period $n$, with $\operatorname{Span}(Q) \subsetneq \operatorname{Span}(P)$. Now, we consider the case $n$ odd, $n>m$. Denote by $I_{i}$ the basic interval with $x_{i}$ as the larger endpoint. Clearly, $I_{0} \rightarrow I_{1} \rightarrow \cdots \rightarrow I_{m-1} \rightarrow I_{0}$ is a loop of length $m$ of the EP-graph of $f$ (cf. the proof of Proposition 5.4). From the definition of undirected orbit it follows that there are two basic intervals adjacent to 0 on branches of different parity, which cover each other. Hence, we can add to our loop of length $m$ the necessary number of times the loop of length two given by these two intervals to get a loop of length $n$. This loop is nonrepetitive and gives a periodic orbit $Q$, of period $n$, with $\operatorname{Span}(Q) \subsetneq \operatorname{Span}(P)$.

We shall need some new definitions and notation.

In case III we denote by $I_{o}$ and $I_{e}$ the basic intervals adjacent to 0 on the odd and even branches, respectively.

In case IV we denote by $I_{o}$ the basic interval adjacent to 0 on the odd branch. $I_{o} f$-covers one of the basic intervals adjacent to 0 on the even branches. We denote this interval by $I_{e}$.

Clearly $I_{o} \rightleftarrows I_{e}$. Set $V_{o}=I_{o} \cup I_{e}$ and $V_{i}=f^{i}\left(V_{o}\right)$ for $i=1,2, \ldots$ We have $V_{o} \subset V_{1}$ and, hence, $V_{i} \subset V_{i+1}$ for all $i$. We denote by $y_{o}, y_{e}$ the endpoints of $I_{o}$ and $I_{e}$, respectively, different from 0 . Then $V_{0}=\operatorname{Span}\left(\left\{y_{o}, y_{e}\right\}\right)$ and, since $f$ is $E P$-adjusted, $V_{i} \supset \operatorname{Span}\left(\left\{f^{j}\left(y_{o}\right): j=0,1, \ldots, i\right\} \cup\left\{f^{j}\left(y_{e}\right)\right.\right.$ : $j=0,1,2, \ldots, i\})$. Since no proper subset of $P$ is invariant, we have $V_{o} \subsetneq$ $V_{1} \subsetneq \cdots \subsetneq V_{r}=\operatorname{Span}(E P)$ for some $r \geq 0$. Since, $\operatorname{Card}\left(V_{o} \cap P\right)=2$, we have $r \leq m-2$.

Lemma 7.3. In case IV, if $P$ is primary, then $P$ has a blue arrow.

Proof. Assume that $P$ is primary but $P$ has only black arrows. Then obviously $m$ is even. Denote by $k$ the smallest integer such that $V_{k} \cap P$ has nonempty intersection with all three branches. We have $k \leq r \leq m-2$. There is a point $x \in P \cap V_{k-1}$ such that $f(x)$ is on that even branch which does not contain $I_{e}$. We may assume that $x$ is the smallest such point. Let $J$ be the basic interval contained in $[0, x]$, adjacent to $x$. Since $P$ has only black arrows, $J$ lies on the odd branch. Clearly, $J f$-covers $I_{e}$. Since $J \subset V_{k-1}$, then either $I_{o}$ or $I_{e} f$-covers $J$ in at most $k-1$ steps. From Lemma 1.12, the $E P$-graph of $f$ has either the loop $I_{o} \rightarrow \cdots \rightarrow J \rightarrow I_{e} \rightarrow I_{o}$ or $I_{e} \rightarrow \cdots \rightarrow J \rightarrow I_{e}$ of length at most $m-1$. However, since all the arrows are black, the lengths of all loops of the EP-graph of $f$ are even. Hence, our loop has length at most $m-2$ (recall that $m$ is even). Adding to our loop the loop $I_{0} \rightleftarrows I_{e}$ enough times, we get a loop of length $m$ going through $I_{e}$ at least two times. This loop is nonrepetitive and, by Lemma 1.3, gives a periodic orbit of $f$ of period $m$. 
Clearly this orbit is different from $P$. From the First Theorem (2.3), we get a contradiction.

Lemma 7.4. Assume that $P$ has a blue arrow. Then $f$ has a pendulum orbit $Q$ of period $l$ odd, $1<l \leq m+1$, such that $\operatorname{Span}(Q) \subset \operatorname{Span}(P)$.

Proof. Let $k$ be the smallest integer such that $V_{k}$ contains a beginning of a blue arrow. We have $k \leq r \leq m-2$.

In case IV, let $J_{e}$ be the basic interval adjacent to 0 on the even branch different from the one on which $I_{e}$ lies. Clearly, $J_{e} f$-covers $I_{o}$.

There is a basic interval $J \subset V_{k}$ such that one of its endpoints is a beginning of a black arrow and the other one is a beginning of a blue arrow. Then $J$ $f$-covers $I_{o}$. Moreover, in case III, $J f$-covers $I_{e}$ and in case IV $J f$-covers $I_{e}$ or $J_{e}$.

Since $J \subset V_{k}$, either $I_{e}$ or $I_{o} f$-covers $J$ in $k$ steps. By Lemma 1.12, there are basic intervals $K_{o}, K_{1}, \ldots, K_{k}$ such that $K_{o}$ is one of $I_{o}, I_{e}, K_{k}=J$ and $K_{i} f$-covers $K_{i+1}$ for $i=0,1, \ldots, k-1$. Clearly, $K_{i} \subset V_{i}$ and, by the minimality of $k$, we obtain $K_{i} \subset \overline{V_{i} \backslash V_{i-1}}$ for $i=1,2, \ldots, k$. Also, by minimality of $k, K_{i}$ and $K_{i+1}$ lie on branches of different parity, for $i=$ $0,1, \ldots, k-1$.

Now in various cases we take various loops of length $l$ :

(i) $k$ even, $K_{o}=I_{o}: I_{o} \rightarrow K_{1} \rightarrow \cdots \rightarrow K_{k} \rightarrow I_{o}, l=k+1$.

(ii) $k$ even, $K_{o}=I_{e}, J f$-covers $I_{e}: I_{e} \rightarrow K_{1} \rightarrow \cdots \rightarrow K_{k} \rightarrow I_{e}, l=k+1$.

(iii) $k$ even, $K_{o}=I_{e}, J f$-covers $J_{e}: I_{e} \rightarrow K_{1} \rightarrow \cdots \rightarrow K_{k} \rightarrow J_{e} \rightarrow I_{o} \rightarrow$ $I_{e}, l=k+3$.

(iv) $k$ odd, $K_{o}=I_{o}, J$ f-covers $I_{e}: I_{o} \rightarrow K_{1} \rightarrow \cdots \rightarrow K_{k} \rightarrow I_{e} \rightarrow I_{o}$, $l=k+2$.

(v) $k$ odd, $K_{o}=I_{o}, J f$-covers $J_{e}: I_{o} \rightarrow K_{1} \rightarrow \cdots \rightarrow K_{k} \rightarrow J_{e} \rightarrow I_{o}$, $l=k+2$.

(vi) $k$ odd, $K_{o}=I_{e}: I_{e} \rightarrow K_{1} \rightarrow \cdots \rightarrow K_{k} \rightarrow I_{o} \rightarrow I_{e}, l=k+2$.

Of course, cases (iii) and (v) can occur only in case IV. Notice that $l$ is always odd and $l \leq k+3 \leq m+1$. Moreover, the loop obtained is nonrepetitive. Hence it gives a periodic orbit $Q$ of period $l$ with $\operatorname{Span}(Q) \subset \operatorname{Span}(P)$.

Assume that $l>3$. We claim that $Q$ is undirected. This is obvious in case III. In case IV, we note that each time we move along a black arrow, we change parity of the branch. Hence, by construction, we change parity of the branch at each step of the loop, except at the step beginning at $K_{k}$. Therefore, if $K_{k}$ is not the closest interval to 0 on its branch among the intervals appearing in the loop, obviously $Q$ is undirected. However, if it is the closest one, by construction, it is the only one on its branch. Hence, $K_{k}$ has to be on an even branch. Thus, we are in case (ii), (iv), or (v) and in the next two steps we go through the intervals closest to 0 on the remaining two branches (during these two steps we cannot come to the branch on which $K_{k}$ is, since $l>3$ ). Then we move in different directions and this proves the claim. 
In both cases (III and IV) the only possibility of not changing parity of the branch when we move along the loop is to make a step from $K_{k}$. Hence, by construction, $Q$ has at most one blue arrow. Since $l$ is odd, it has exactly one blue arrow. Thus, $Q$ satisfies condition (i) of Definition 4.1 for the odd case.

Observe that always, when we use some other intervals than $K_{1}, K_{2}, \ldots$, $K_{k}$, they are $I_{o}, I_{e}, J_{e}$. Moreover, each of them is used at most once and they appear in the loop following $K_{k}$ and preceding $K_{1}$. Since $I_{o}, I_{e}, J_{e}$ are adjacent to 0 (each on its branch) and $K_{i} \in \overline{V_{i} \backslash V_{i-1}}$, we obtain condition (ii) of Definition 4.1 for the odd case. This proves the lemma in the case $l>3$.

If $l=3$ then we add to our loop the loop $I_{o} \rightleftarrows I_{e}$. Then we obtain a loop of length 5 and an associated periodic orbit $\tilde{Q}$ of period $\tilde{l}=5$ with $\operatorname{Span}(\tilde{Q}) \subset \operatorname{Span}(P)$. Obviously it is undirected and has exactly one blue arrow. If we have in our loop intervals $I \in\left\{I_{o}, I_{e}, J_{e}\right\}$ and $K \in\left\{K_{1}, K_{2}\right\}$ lying on the same branch, then $I$ is closer to 0 than $K$. Since $f$ is $E P$-adjusted this proves condition (ii) of Definition 4.1 for the odd case. This ends the proof if $m \geq 4$.

If $m=2$, then $P$ has only black arrows, a contradiction.

If $m=3$, then $P$ is pendulum and we can take $Q=P$.

Lemma 7.5. Let $P$ be a primary orbit of an EP-adjusted map $f \in \mathbf{X}$. Assume that $P$ is undirected and has period $m \geq 2$. Then,

(a) If $m$ is even, then all arrows are black and we have case III.

(b) If $m$ is odd, then $P$ is pendulum.

Remark 7.6. One should remember that cases I and II were reduced to case III. So, in fact, cases I and II may occur under the assumptions of (a) of Lemma 7.5 as well as case III.

Proof of Lemma 7.5. (a) Assume that $P$ has a blue arrow. From Lemma $7.4 f$ has a pendulum orbit $Q$ of period $l$ odd, such that $\operatorname{Span}(Q) \subset \operatorname{Span}(P)$. Let $h$ be an $E Q$-adjusted map. By Lemma 7.2, $h$ has a periodic orbit $R$ of period $m$ such that $\operatorname{Span}(R) \subsetneq \operatorname{Span}(Q)$. From the Adjusting Lemma (1.18), $f$ has a periodic orbit $\tilde{R} \quad E$-equivalent to $R$, such that $\operatorname{Span}(\tilde{R}) \subsetneq \operatorname{Span} Q$. By the First Theorem (2.3), this is a contradiction. Hence, $P$ has all arrows black. By Lemma 7.3, we have case III.

(b) Since $m$ is odd, $P$ has a blue arrow. By Lemma 7.4, $f$ has a pendulum orbit $Q$ of period $l$ odd, $1<l \leq m+1$, such that $\operatorname{Span}(Q) \subset \operatorname{Span}(P)$. Since both $l$ and $m$ are odd, $l \leq m$. If $l<m$ then, by Lemma 7.2 and the Adjusting Lemma, $f$ has a periodic orbit $R$ of period $m$ such that $\operatorname{Span}(R) \subsetneq \operatorname{Span}(P)$. By the First Theorem, this is a contradiction. Hence, $l=m$. Again, by the First Theorem, $Q=P$ because $P$ is primary. Hence $P$ is pendulum.

\section{Primary extensions}

In this section we study some properties of extensions of primary orbits. Throughout the section we shall assume that $P$ is a periodic orbit of a map 
$f \in \mathbf{X}$ and $P$ is an $n$-extension of $Q$. We also use the notation of Definitions 3.1 and 3.3 , simplified by assuming that $\psi(i)=i+1(\bmod s)$ (we renumerate $P_{i}$ 's if necessary). In this section we shall not take into account whether $P$ is directed or undirected.

In fact, the results of this section and other results about extensions are fairly general and apply to a large class of maps of one-dimensional spaces (cf. [BCJM]). We denote the elements of $P_{i}$ by $x_{0}^{i}, x_{1}^{i}, \ldots, x_{n-1}^{i}$ in such a way that $f^{s}\left(x_{j}^{i}\right)=x_{j+1}^{i}(\bmod n)$. When using this notation we shall keep the convention that addition at subindices of $P$ and superindices of $x$ is mod $s$ and at subindices of $x$ is $\bmod n$.

Lemma 8.1. If $f^{p}\left(x_{j}^{i}\right)=x_{l}^{i+p}$, then $f^{p}\left(x_{j+q}^{i}\right)=x_{l+q}^{i+p}$, for $q=1,2, \ldots, n-1$. Proof. $f^{p}\left(x_{j+q}^{i}\right)=f^{p+q s}\left(x_{j}^{i}\right)=f^{q s}\left(x_{l}^{i+p}\right)=x_{l+q}^{i+p}$.

Lemma 8.2. Let $P$ be primary and let $n$ be even. Then $P$ is an $n / 2$-extension of a 2-extension of $Q$.

Proof. By Lemma 3.13, each $P_{i}$ is a primary orbit of $f^{s}$. Denote $P_{i}^{o}=\left\{x_{j}^{i}: j\right.$ is odd $\}, P_{i}^{e}=\left\{x_{j}^{i}: j\right.$ even $\}$. Since $\operatorname{Span}\left(P_{i}\right)$ is an interval, from Lemma 7.5(a) and Remark 7.6 it follows that $\operatorname{Span}\left(P_{i}^{o}\right) \cap \operatorname{Span}\left(P_{i}^{e}\right)=\varnothing$ (see also Remark 5.1). From Lemma 8.1, we have $\left\{f\left(P_{i}^{o}\right), f\left(P_{i}^{e}\right)\right\}=\left\{P_{i+1}^{o}, P_{i+1}^{e}\right\}$ for each $i$. Hence, $P$ is an $n / 2$-extension of a 2-extension of $Q$, where this 2-extension of $Q$ is obtained by collapsing each of the sets $P_{i}^{o}, P_{i}^{e}$ to a point (as in (iv) of Definition 3.1).

Lemma 8.3. Let $P$ be primary, $n$ odd, and $n \geq 3$. Then for each $i$ we can renumerate $x_{j}^{i}$ 's in such a way that still $f^{s}\left(x_{j}^{i}\right)=x_{j+1}^{i}$ and either

$$
\cdots x_{7}^{i}<x_{5}^{i}<x_{3}^{i}<x_{1}^{i}<x_{0}^{i}<x_{2}^{i}<x_{4}^{i}<x_{6}^{i}<\cdots
$$

or

$$
\cdots x_{7}^{i}>x_{5}^{i}>x_{3}^{i}>x_{1}^{i}>x_{0}^{i}>x_{2}^{i}>x_{4}^{i}>x_{6}^{i}>\cdots .
$$

Proof. By Lemma 3.13, each $P_{i}$ is a primary orbit of $f^{s}$. Since $\operatorname{Span}\left(P_{i}\right)$ is an interval, from Lemma 7.5(b), Remark 7.6, and Definition 4.1, there is a point $e_{i} \in \operatorname{Span}\left(P_{i}\right)$ such that (perhaps after renumeration of $x_{j}^{i}$ 's, which keeps the property that $\left.f^{s}\left(x_{j}^{i}\right)=x_{j+1}^{i}\right)$ all $x_{j}^{i}$ 's with $j$ even are on the same side of $e_{i}$, and all $x_{j}^{i}$ 's with $j$ odd are on the other side of $e_{i}$. Moreover, we know that $x_{j}^{i} \in\left(e_{i}, x_{k}^{i}\right)$ is equivalent to $j<k$. From this the statement of the lemma follows easily.

In the rest of this section we shall use the assumptions, results, and notation of Lemma 8.3.

Lemma 8.4. Under the assumptions of Lemma 8.3, if $p \leq s$ then between $f^{p}\left(x_{0}^{i}\right)$ and $f^{p}\left(x_{1}^{i}\right)$ there are no points $f^{p}\left(x_{j}^{i}\right)$ for $j<n-1$. 
Proof. We may assume that $f$ is $E P$-adjusted. Suppose that $f^{p}\left(x_{j}^{i}\right) \in$ $\left(f^{p}\left(x_{0}^{i}\right), f^{p}\left(x_{1}^{i}\right)\right)$ for some $j<n-1$. Clearly $j>1$. Then

$$
\begin{aligned}
f^{s}\left(\left[x_{0}^{i}, x_{1}^{i}\right]\right) & \supset f^{s-p}\left(\left[f^{p}\left(x_{0}^{i}\right), f^{p}\left(x_{1}^{i}\right)\right]\right) \supset f^{s-p}\left(\left[f^{p}\left(x_{0}^{i}\right), f^{p}\left(x_{j}^{i}\right)\right]\right) \\
& \supset\left[f^{s}\left(x_{0}^{i}\right), f^{s}\left(x_{j}^{i}\right)\right]=\left[x_{1}^{i}, x_{j+1}^{i}\right] \supset\left[x_{j-1}^{i}, x_{j+1}^{i}\right] .
\end{aligned}
$$

Thus, in the $P_{i}$-graph of $f^{s}$ we have the loop

$$
\left[x_{0}^{i}, x_{1}^{i}\right] \rightarrow\left[x_{j-1}^{i}, x_{j+1}^{i}\right] \rightarrow\left[x_{j}^{i}, x_{j+2}^{i}\right] \rightarrow \cdots \rightarrow\left[x_{n-2}^{i}, x_{n}^{i}\right] \rightarrow\left[x_{0}^{i}, x_{1}^{i}\right]
$$

of length smaller than $n$. With addition of the loop $\left[x_{0}^{i}, x_{1}^{i}\right] \rightarrow\left[x_{0}^{i}, x_{1}^{i}\right]$ repeated enough times, we get a periodic orbit $P_{i}^{\prime}$ of $f^{s}$ of period $n$ with $\operatorname{Span}\left(P_{i}^{\prime}\right) \subsetneq \operatorname{Span}\left(P_{i}\right)$. By Lemma 3.9 and the First Theorem (2.3), this is a contradiction.

Lemma 8.5. Under the assumptions of Lemma 8.3, if $n>3$ and $p \leq s$, then either $f^{p}\left(x_{0}^{i}\right)=x_{0}^{i+p}$ or $f^{p}\left(x_{0}^{i}\right)=x_{1}^{i+p}$.

Proof. Let $f^{p}\left(x_{0}^{i}\right)=x_{j}^{i+p}$. Then, by Lemma 8.1, $f^{p}\left(x_{1}^{i}\right)=x_{j+1}^{i+p}$. By Lemma 8.4, there are two possibilities:

1. $P \cap\left(x_{j}^{i+p}, x_{j+1}^{i+p}\right)=\varnothing$. Then, from the ordering of the points $x_{l}^{i+p}$, $l=0,1,2, \ldots, n-1$, we get $j=0$ (here we use the assumption that $n>3$ ).

2. $P \cap\left(x_{j}^{i+p}, x_{j+1}^{i+p}\right)$ has exactly one element. Then, from the ordering of the points $x_{l}^{i+p}, l=0,1,2, \ldots, n-1$, we get $j=1$.

Lemma 8.6. Under the assumptions of Lemma 8.3, there is exactly one $t<s$ such that $f\left(x_{0}^{t}\right) \neq x_{0}^{t+1}$.

Proof. Since $f^{s}\left(x_{0}^{t}\right)=x_{1}^{t}$, such $t$ exists. Suppose that there are $t, r<s, t \neq r$, such that $f\left(x_{0}^{t}\right) \neq x_{0}^{t+1}$ and $f\left(x_{0}^{r}\right) \neq x_{0}^{r+1}$. We may assume that $r<t$ and that $t$ is the smallest integer larger than $r$ such that $f\left(x_{0}^{t}\right) \neq x_{0}^{t+1}$.

Assume that $n>3$. By Lemmas 8.1 and 8.5 , we have $f^{t-r+1}\left(x_{0}^{r}\right)=x_{2}^{t+1}$. Since $t-r+1 \leq s$, this contradicts Lemma 8.5.

Assume that $n=3$. We may assume that $f$ is $E P$-adjusted. In each $\operatorname{Span}\left(P_{i}\right)$ we have two basic intervals: $I^{i}$ and $J^{i}$. One of the intervals $I^{r}, J^{r}$ $f$-covers both $I^{r+1}, J^{r+1}$. Analogously, one of $I^{t}, J^{t} f$-covers both $I^{t+1}$, $J^{t+1}$. Clearly, for all $i$, either $I^{i} f$-covers $I^{i+1}$ and $J^{i} f$-covers $J^{i+1}$, or $I^{i}$ $f$-covers $J^{i+1}$ and $J^{i} f$-covers $I^{i+1}$. Hence, one of the intervals $I^{s}, J^{s}$ (say $\left.I^{s}\right) f^{s}$-covers one of the intervals $I^{s}, J^{s}$ at least twice and the other one at

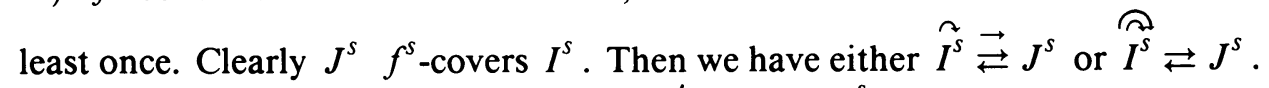
In both cases we obtain a periodic orbit $P_{s}^{\prime} \neq P_{s}$ of $f^{s}$ of period 3 (here we use generalized graphs, cf. [BGMY]; the reader will easily see how we get period 3). By Lemma 3.9 and the First Theorem (2.3), this is a contradiction. 


\section{UNDIRECTED THEOREM (SECOND PART)}

In this section we complete the proof of the Undirected Theorem (7.1). To do this we use the preliminary results given in $\S \S 7$ and 8 .

Lemma 9.1. Assume that $P$ is a periodic orbit of an EP-adjusted map $f \in \mathbf{X}$ of period $m=2^{k} . n, k>0$. Assume also that $P$ is primary and undirected. Then $P$ is an n-extension of $Q$, where $Q$ is a pendulum orbit of period $2^{k}$.

Proof. By the General Rule (6.1), we have case I, II, III, or IV (we use the notation of $\S 4$ ). Hence, as in $\S 7$, from Remark 5.1 , we may assume that the case is III or IV.

We use induction. From Lemma 7.5(a) we have case III and all arrows are black. Hence, $P$ is a $2^{k-1} . n$-extension of some orbit $Q_{1}$ of period 2. Clearly, $Q$ is pendulum. Now, assume that $P$ is a $2^{k-l} \cdot n$-extension of a pendulum orbit $Q_{l}$ of period $2^{l}$, and $1 \leq l<n$. Then, by Lemma $8.2, P$ is a $2^{k-(l+1)} \cdot n$ extension of some orbit $Q_{l+1}$ which is a 2-extension of $Q_{l}$. By Definition 4.1, $Q_{l+1}$ is a pendulum orbit of period $2^{l+1}$. This finishes the induction.

Proof of Undirected Theorem (7.1). Again, by General Rule (6.1) and Remark 5.1 , we may assume that the case is III or IV. Also, we may assume that $f$ is $E P$-adjusted. Let $m$ be the period of $P$. If $m$ is odd, we use Lemma 7.5(b). If $m=2^{k}, k>0$, we use Lemma 9.1 with $n=1$. If $m=2^{k} . n, n$ is odd, $n \geq 3$, and $k>0$, then, by Lemma 9.1, $P$ is an $n$-extension of a pendulum orbit $Q$ of period $2^{k}$. By Lemmas 8.1, 8.3, and 8.6, condition (vi) of Definition 3.3 is satisfied. With the notation of $\S \S 3$ and 7 , we set $R=P_{o}$ and $g=f^{2^{k}}$. By Lemma 3.13, $R$ is primary. Since the period of $R$ is odd and larger than one, by Lemma 7.5(b) and Remark 5.1, $R$ is pendulum. Hence by Definitions 3.3 and 4.1, $P$ is pendulum.

Remark 9.2. By Proposition 5.4, the Undirected Theorem (7.1), and Remark 4.18 , in the case of maps of the interval, all primary orbits are pendulum and vice versa. Thus, if $P$ is a periodic orbit of period $m$ of a $P$-adjusted map $f \in I$ and $P$ is primary, then it is minimal. Moreover, the converse is true if $m$ is not of the form $3.2^{i}, i \geq 1$ (see [C, ALS, or H]. We can understand the relation between the ideas of minimality and primarity as follows. We can say that a periodic orbit $P$ forces a periodic orbit $Q$ if an $E P$-adjusted map has a periodic orbit different from $P$ and $E$-equivalent to $Q$. Let $P$ be a periodic orbit of $f \in \mathbf{X}$ of period $m$. Then $P$ is primary if $P$ does not force any orbit of period $m . P$ is minimal if $P$ does not force any orbit of period different from $m$ which in turn forces a different periodic orbit of period $m$. This shows that these notions are indeed close to each other.

As a corollary to Remark 9.2 and the Primary Theorem (2.5) We obtain the following result of Block and Coppel.

Theorem 9.3 (see [BC]). If $f \in \mathbf{I}$ and $n \in \operatorname{Per}(f)$, then $f$ has as simple periodic orbit of period $n$. 


\section{Directed Theorem}

In this section we prove the following result (we use the notation of $\S 4$ and all congruences are mod 3 ).

Theorem 10.1 (Directed Theorem). Let $P$ be a periodic orbit of a map $f \in \mathbf{Y}$. If $P$ is directed and primary, then the following hold.

(a) If $P$ has only black arrows, then $P$ is twist.

(b) $P$ cannot have both red and green arrows.

(c) If $P$ has only one green (red) arrow, then $P$ is single green (resp. red).

(d) $P$ cannot have more than two colored arrows.

(e) If $P$ has two green (red) arrows and its period is even, then $P$ is box green (resp. red).

(f) If $P$ has two green (red) arrows and its period is odd, then $P$ is double green (resp. red).

We note that, to prove the Directed Theorem, we may assume that $f$ is $E P$ adjusted. Hence, in the rest of the section we assume that $P$ is a directed orbit of an $E P$-adjusted map $f \in \mathbf{Y}$ of period $m$. Since $P$ is directed, $m \geq 3$. We also assume that $P$ is primary.

The following is analogous to Lemma 9.1.

Lemma 10.2. If $P$ has only black arrows and $m=3.2^{k} . n, k \geq 0$, then $P$ is an $n$-extension of $Q$ for some twist orbit $Q$ of period $3.2^{k}$.

Proof. We use induction. Clearly $P$ is a $2^{k} n$-extension of some twist orbit $Q_{0}$ of period 3. If $P$ is a $3.2^{k-l} \cdot n$-extension of a twist orbit $Q_{l}$ of period $3.2^{l}$ and $0 \leq l<k$ then, by Lemma $8.2, P$ is a $3.2^{k-(l+1)} \cdot n$-extension of some orbit $Q_{l+1}$ which is a 2-extension of $Q_{l}$. By Definition 4.40, $Q_{l+1}$ is a twist orbit of period $3.2^{l+1}$. This finishes the proof.

Proof of (a) of Directed Theorem (10.1). If $m=3.2^{k}$, we use Lemma 10.2 with $n=1$. If $m=3.2^{k} . n, n$ odd, $n \geq 3$, then by Lemma $10.2, P$ is an $n$-extension of a twist orbit $Q$ of period $3.2^{k}$. By Lemmas 8.6, 8.3, and 8.1, condition (vi) of Definition 3.3 is satisfied. We set $R=P_{0}$ and $g=f^{3.2^{k}}$. By Lemma 3.13, $R$ is primary. By the Undirected Theorem (7.1), $R$ is pendulum. Hence, by Definitions 8.3 and 4.40, $P$ is twist.

Now we define the sets $V_{0}, V_{1}, V_{2}, \ldots$ as follows.

$$
V_{0}=\operatorname{Span}\left(\left\{\mathrm{sm}_{0}, \mathrm{sm}_{1}, \mathrm{sm}_{2}\right\}\right)
$$

and $V_{i}=f^{i}\left(V_{0}\right)$ for $i=1,2, \ldots$. We have $V_{0} \subset V_{1}$ and hence $V_{i} \subset V_{i+1}$ for each $i$. Since $f$ is $E P$-adjusted, $V_{i} \supset \operatorname{Span}\left(\left\{f^{j}\left(\operatorname{sm}_{k}\right): j=0,1, \ldots, i ; k=\right.\right.$ $0,1,2\})$. Since no proper subset of $P$ is invariant, we have $V_{i+1} \neq V_{i}$ unless $V_{i}=\operatorname{Span}(P)$. Hence, $V_{0} \subsetneq V_{1} \subsetneq V_{2} \subsetneq \cdots \subsetneq V_{r-1} \subsetneq V_{r}=\operatorname{Span}(P)$ for some $r \geq 0$. Since $\operatorname{Card}\left(V_{0} \cap P\right)=3$, we have $r \leq m-3$. 
Lemma 10.3. Let $k$ be the smallest integer such that there exist arrows $A$ and $B$ such that $b(B) \leq e(A), b(A) \leq e(B)$, and $b(A) \in V_{k}$ or $b(B) \in V_{k}$; or let $k=r$ if such arrows do not exist. Let $i \leq k$. If a colored arrow $C$ begins in $V_{i}$ then it also ends in $V_{i}$.

Proof. Suppose the lemma is not true. Then we can take the smallest $i \leq k$ such that there exists a colored arrow $C$ which begins in $V_{i}$ and does not end in $V_{i}$. Since $V_{i}=\operatorname{Span}\left(V_{i}\right)$, by the General Rule (6.1), $C$ cannot be green. Hence it is red. Let $x$ be the largest point of $V_{i}$ on the same branch as $b(C)$. Then $x=e(D)$ for some arrow $D$ such that $b(D) \in V_{i-1} \quad(i>0$ since all arrows beginning in $V_{o}$ are black). $D$ cannot be black since then we would have $b(D)<e(C), b(C) \leq e(D)$, and $b(D) \in V_{i-1} \subset V_{k-1}$, a contradiction with the minimality of $k$. This contradicts the minimality of $i$.

Lemma 10.4 (Directed Rule). There are no arrows $A$ and $B$ such that $b(B) \leq$ $e(A)$ and $b(A) \leq e(B)$.

Proof. We assume the Directed Rule does not hold. Then we can take $A, B$, and $k$ as in the hypotheses of Lemma 10.3. Since $A$ and $B$ have opposite directions, one of them is red and the other is black. We may assume that $A$ is red and $B$ is black. We may also assume that ind $(b(A))=0$ (see Figure 10.5).

We set $I_{i}=\left[0, \mathrm{sm}_{i}\right]$ for $i=0,1,2, K=\left[\mathrm{sm}_{0}, b(A)\right]$ (since $A$ is red, $\left.\mathrm{sm}_{0} \neq b(A)\right), L=[0, b(B)]$. We have the graph (i) shown in Figure 10.5. Since $b(A) \in V_{k}$ or $b(B) \in V_{k}$, we have $K \subset V_{k}$ or $L \subset V_{k}$, respectively.
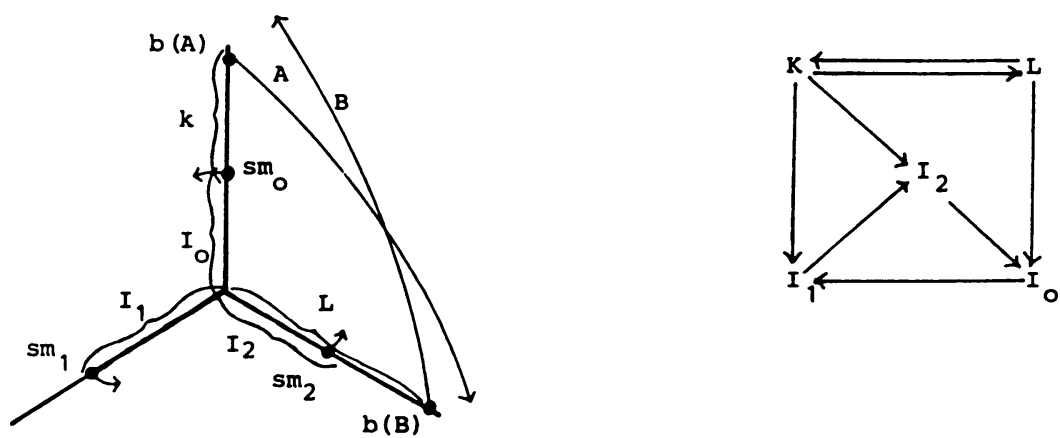

Figure 10.5 .

If $e(B)>b(A)$ then set $x=e(B)$. If not, then $e(A)>b(B)$ and we set $x=e(A)$. We have $x \notin V_{k-1}$, by the minimality of $k$. If $r=m-3$, then $\operatorname{Card}\left(\left(V_{i+1} \backslash V_{i}\right) \cap P\right)=1$ for $i=0,1,2, \ldots, r-1$, and we have $x \notin V_{k}$.

We claim that $k \leq m-4$. To see this consider two cases. If $r<m-3$, then $k \leq r \leq m-4$. If $r=m-3$, then $x \notin V_{k}$. Hence, $k<r$.

Notice that $x \in P \backslash\left(I_{0} \cup I_{1} \cup I_{2} \cup K \cup L\right)$.

One of the intervals $I_{0}, I_{1}, I_{2}$ (call it $J_{0}$ ) covers one of the intervals $K$, $L$ (call it $J_{k}$ ) in $k$ steps. Since $x \notin V_{k-1}$, we have $x \notin J_{0}, x \notin f\left(J_{0}\right)$, $x \notin f^{2}\left(J_{0}\right), \ldots, x \notin f^{k-1}\left(J_{0}\right), x \notin J_{k}$. Therefore, for each loop obtained from graph (i) and the path $J_{0} \rightarrow f\left(J_{0}\right) \rightarrow f^{2}\left(J_{0}\right) \rightarrow \cdots \rightarrow f^{k-1}\left(J_{0}\right) \rightarrow J_{k}$, 
of length $k$, the associated periodic orbit does not contain $x$. Hence, this orbit is different from $P$. This is true also for $k=0$ (i.e., the case when $J_{0}=I_{2}=L=J_{k}$ ).

By Lemma 10.3 , instead of sets $f\left(J_{0}\right), f^{2}\left(J_{0}\right), \ldots, f^{k-1}\left(J_{0}\right)$ we can take the sets $J_{1}, J_{2}, \ldots, J_{k-1}$ where $J_{i}=\left[0, y_{i}\right]$ and $y_{i}$ is the largest point of $V_{i}$ on the branch next after the one on which $J_{i-1}$ lies. Then we still have

$$
J_{0} \rightarrow J_{1} \rightarrow J_{2} \rightarrow \cdots \rightarrow J_{k-1} \rightarrow J_{k} .
$$

This is because Lemma 10.3 states that, for $i \leq k$, the increment of $V_{i+1}$ with respect to $V_{i}$ is due to black arrows. We note that still $x \notin J_{i}$ for $i=0,1,2, \ldots, k$.

Now we are going to construct a loop $\alpha$ of length $m$ by using path (ii), some path of the graph (i), and path $K \rightarrow L$ repeated $l$ times. We have to consider several cases.

(1) $J_{0}=I_{0}$ and $J_{k}=K$.

1.1. $m-k$ even, $l=(m-k) / 2$, and

$$
\alpha=I_{0} \rightarrow J_{1} \rightarrow \cdots \rightarrow J_{k-1} \rightarrow K \rightarrow L \rightarrow K \rightarrow L \rightarrow \cdots \rightarrow K \rightarrow L \rightarrow I_{0} .
$$

1.2. $m-k$ odd, $l=(m-k-3) / 2$, and

$$
\begin{aligned}
\alpha= & K \rightarrow I_{1} \rightarrow I_{2} \rightarrow I_{0} \rightarrow J_{1} \rightarrow \cdots \\
& \rightarrow J_{k-1} \rightarrow K \rightarrow L \rightarrow K \rightarrow L \rightarrow \cdots \rightarrow K \rightarrow L \rightarrow K .
\end{aligned}
$$

(2) $J_{0}=I_{0}$ and $J_{k}=L$.

2.1. $m-k$ even, $l=(m-k-4) / 2$, and

$$
\begin{aligned}
\alpha= & K \rightarrow I_{1} \rightarrow I_{2} \rightarrow I_{0} \rightarrow J_{1} \rightarrow \cdots \rightarrow J_{k-1} \\
& \rightarrow L \rightarrow K \rightarrow L \rightarrow K \rightarrow L \rightarrow \cdots \rightarrow K \rightarrow L \rightarrow K .
\end{aligned}
$$

2.2. $m-k$ odd, $l=(m-k-1) / 2$, and $\alpha=I_{0} \rightarrow J_{1} \rightarrow \cdots \rightarrow J_{k-1} \rightarrow L \rightarrow K \rightarrow L \rightarrow K \rightarrow L \rightarrow \cdots \rightarrow K \rightarrow L \rightarrow I_{0}$.

(3) $J_{0}=J_{1}$ and $J_{k}=K$.

3.1. $m-k$ even, $l=(m-k-4) / 2$, and

$$
\begin{aligned}
\alpha= & K \rightarrow I_{1} \rightarrow I_{2} \rightarrow I_{0} \rightarrow I_{1} \rightarrow J_{1} \rightarrow \cdots \\
& \rightarrow J_{k-1} \rightarrow K \rightarrow L \rightarrow K \rightarrow L \rightarrow \cdots \rightarrow K \rightarrow L \rightarrow K .
\end{aligned}
$$

3.2. $m-k$ odd, $l=(m-k-1) / 2$, and

$$
\alpha=K \rightarrow I_{1} \rightarrow J_{1} \rightarrow \cdots \rightarrow J_{k-1} \rightarrow K \rightarrow L \rightarrow K \rightarrow L \rightarrow \cdots \rightarrow K \rightarrow L \rightarrow K .
$$

(4) $J_{0}=I_{1}$ and $J_{k}=L$.

4.1. $m-k$ even, $l=(m-k-2) / 2$, and

$$
\begin{aligned}
\alpha= & K \rightarrow I_{1} \rightarrow J_{1} \rightarrow \cdots \rightarrow J_{k-1} \\
& \rightarrow L \rightarrow K \rightarrow L \rightarrow K \rightarrow L \rightarrow \cdots \rightarrow K \rightarrow L \rightarrow K .
\end{aligned}
$$


4.2. $m-k$ odd, $l=(m-k-5) / 2$, and

$$
\begin{aligned}
\alpha= & K \rightarrow I_{1} \rightarrow I_{2} \rightarrow I_{0} \rightarrow I_{1} \rightarrow J_{1} \rightarrow \cdots \rightarrow J_{k-1} \\
& \rightarrow L \rightarrow K \rightarrow L \rightarrow K \rightarrow L \rightarrow \cdots \rightarrow K \rightarrow L \rightarrow K .
\end{aligned}
$$

(5) $J_{0}=I_{2}$ and $J_{k}=K$.

5.1. $m-k$ even, $l=(m-k-2) / 2$, and

$$
\begin{aligned}
\alpha= & K \rightarrow I_{1} \rightarrow I_{2} \rightarrow J_{1} \rightarrow \cdots \\
& \rightarrow J_{k-1} \rightarrow K \rightarrow L \rightarrow K \rightarrow L \rightarrow \cdots \rightarrow K \rightarrow L \rightarrow K .
\end{aligned}
$$

5.2. $m-k$ odd, $l=(m-k-5) / 2$, and

$$
\begin{aligned}
\alpha= & K \rightarrow I_{1} \rightarrow I_{2} \rightarrow I_{0} \rightarrow I_{1} \rightarrow I_{2} \rightarrow J_{1} \rightarrow \cdots \\
& \rightarrow J_{k-1} \rightarrow K \rightarrow L \rightarrow K \rightarrow L \rightarrow \cdots \rightarrow K \rightarrow L \rightarrow K .
\end{aligned}
$$

(6) $J_{0}=I_{2}$ and $J_{k}=L$

6.1. $m-k$ even, $l=(m-k-2) / 2$, and

$$
\begin{aligned}
& \alpha=K \rightarrow I_{2} \rightarrow J_{1} \rightarrow \cdots \rightarrow J_{k-1} \\
& \rightarrow L \hookrightarrow K \rightarrow L \rightarrow K \rightarrow L \rightarrow \cdots \rightarrow K \rightarrow L \rightarrow K .
\end{aligned}
$$

6.2. $m-k$ odd, $l=(m-k-3) / 2$, and

$$
\begin{aligned}
\alpha= & I_{0} \rightarrow I_{1} \rightarrow I_{2} \rightarrow J_{1} \rightarrow \cdots \rightarrow J_{k-1} \\
& \rightarrow L \rightarrow K \rightarrow L \rightarrow K \rightarrow L \rightarrow \cdots \rightarrow K \rightarrow L \rightarrow I_{0} .
\end{aligned}
$$

(7) $k=0$. Then $I_{2}=L$ and $m \geq 5$.

7.1. $m$ even, $l=(m-6) / 2$, and

$$
\alpha=K \rightarrow I_{1} \rightarrow L \rightarrow I_{0} \rightarrow I_{1} \rightarrow L \rightarrow K \rightarrow L \rightarrow K \rightarrow L \rightarrow \cdots \rightarrow K \rightarrow L \rightarrow K .
$$

7.2. $m$ odd, $l=(m-3) / 2$, and

$$
\alpha=K \rightarrow I_{1} \rightarrow L \rightrightarrows K \rightarrow L \rightarrow K \rightarrow L \rightarrow \cdots \rightarrow K \rightarrow L \rightarrow K .
$$

We note that since $k \leq m-4$ in cases $1.1,1.2,2.2,3.2,4.1,5.1,6.1,6.2$, and 7.2 we have $l>0$. Hence, in all cases $\alpha$ goes through $K$. Thus, the periodic orbit associated to $\alpha$ is different from $\{0\}$.

Notice that in graph (i) with path (ii) we always move to the next branch, except when we go from $K$ to $L$ or $I_{2}$. In this case we move to the previous branch. Hence, if $l>0$, inspection of cases 1-7 assures us that the periodic orbit associated to $\alpha$ has period $m$. Hence, we are left with the case $l=0$. Then we have one of the cases 2.1, 3.1, 4.2, 5.2, and 7.1. Notice that $\alpha$ goes through $I_{0}$ and, when we move along $\alpha$, each time we move to the next branch. Hence, $m \equiv 0$ and the periodic orbit associated to $\alpha$ has only black arrows. Thus, its period is $3 . n, n \geq 1$, and $3 n \mid m$. Clearly, this orbit is an $n$-extension of a twist orbit of period three. Therefore, it we look at $f^{3}$ restricted to $\mathrm{br}_{0}$ we have a periodic orbit of $f^{3}$ of period $n$. Clearly $n \mid m / 3$. Hence, if $m$ 
is odd, then $n$ is odd. Moreover, $n>1$ since the orbit has points in both $K$ and $I_{0}$. Thus $n<_{s} m / 3$.

If $m$ is even, then we add to $\alpha$ the loop $I_{0} \rightarrow I_{1} \rightarrow I_{2} \rightarrow I_{0}$ and we get a new loop of length $m+3$. We repeat the above process for the periodic orbit associated to this new loop.

Summarizing, in the case $l=0$, we have a periodic orbit $Q$ of period 3.n, $n$ odd, $n \geq 3$, which is an $n$-extension of a twist orbit of period three, where $n<_{s} m / 3$. Clearly $x \notin Q$ and then $\operatorname{Span}(Q) \subsetneq \operatorname{Span}(P)$.

Let $h$ be an $E Q$-adjusted map. If we look at $h^{3}, Q \cap \mathrm{br}_{0}$ is a periodic orbit of $h^{3}$ of period $n$. By the Primary Theorem (2.5), $h^{3}$ has a primary periodic orbit $Q^{\prime}$ of period $n$ such that $\operatorname{Span}\left(Q^{\prime}\right) \subset \operatorname{Span}\left(Q \cap \mathrm{br}_{0}\right)$. By the Undirected Theorem (7.1) this orbit is pendulum. Let $g$ be a $Q^{\prime}$ adjusted map. By Lemma 7.2, $g$ has a periodic orbit $Q^{\prime \prime}$ of period $m / 3$ such that $\operatorname{Span}\left(Q^{\prime \prime}\right) \subsetneq \operatorname{Span}\left(Q^{\prime}\right)$. By the Adjusting Lemma (1.18) $h^{3}$ has a periodic orbit of period $m / 3$ with span strictly contained in $\operatorname{Span}\left(Q^{\prime}\right) \subset \operatorname{Span}\left(Q \cap \mathrm{br}_{0}\right)$. By Lemma 3.9, $h$ has a periodic orbit of period $m$ with span contained in $\operatorname{Span}(Q)$. By the Adjusting Lemma, $f$ has a periodic orbit of period $m$ different from $P$.

In all cases, we have a periodic orbit of period $m$ different from $P$. By the First Theorem (2.3) this is a contradiction with the primarity of $P$.

Corollary 10.6. If a colored arrow begins in $V_{i}$, it also ends in $V_{i}$.

Proof. By the Directed Rule (10.4), in Lemma 10.3 we have $k=r$. Hence, the statement of the corollary holds for $i \leq r$. However, if $i>r$, then $V_{i}=V_{r}$.

Lemma 10.7. Let $P$ be a single green (red) orbit of period $n$ of an EP-adjusted map $f \in \mathbf{Y}$. Then $f$ has:

(a) Single green (resp. red) orbits of periods $m+3, m+6, m+9, \ldots$.

(b) Twist orbits of periods $3,6,9, \ldots$.

Proof. To prove (a) it is enough to prove that $f$ has a single green (resp. red) orbit of period $m+3$ (then one can use induction).

We use the notation of Definition 4.19. Set $I_{i}=\left[0, x_{i}\right]$ for $i=0,1,2$ and $I_{i}=\left[x_{i-3}, x_{i}\right]$ for $i=3,4, \ldots, m-1$. We have the loop $I_{0} \rightarrow I_{1} \rightarrow I_{2} \rightarrow$ $I_{0} \rightarrow I_{1} \rightarrow \cdots \rightarrow I_{m-1} \rightarrow I_{0}$ of length $m+3$ in the EP-graph of $f$. Clearly this loop is nonrepetitive and, by Lemma 1.3, $f$ has a periodic orbit $Q$ of period $m+3$ associated to it. $Q$ has two points in each of the intervals $I_{i}$, $i=0,1,2$. Since $f$ is $E P$-adjusted, the point of $I_{i} \cap Q$ which needs a longer time to get to $I_{3}$ is smaller than the other one. Hence $Q$ is single green (resp. red).

To prove (b), we consider the loop $I_{m-3} \rightarrow I_{m-2} \rightarrow I_{m-1} \rightarrow I_{m-3}$ and

$$
\begin{array}{ll}
\text { if } m>6: & I_{m-6} \rightarrow I_{m-5} \rightarrow \cdots \rightarrow I_{m-1} \rightarrow I_{m-6} \\
\text { if } m<6: & I_{0} \rightarrow I_{1} \rightarrow I_{2} \rightarrow I_{0} .
\end{array}
$$

All steps in these loops are made to the next branch. Combining these loops (repeating them if necessary) we can obtain a nonrepetitive loop of length $n$ 
for every $n$ such that $3 \mid n$. By Lemma 1.3, this loop has associated a periodic orbit $Q_{n}$ of period $n . Q_{n}$ is directed and has all arrows black. Let us take an $E Q_{n}$-adjusted map $h$. By the Primary Theorem (2.5), $h$ has a primary orbit $R$ of period $n$ with all arrows black. Hence, by the Directed Theorem (10.1)(i), $R$ is twist. By the Adjusting Lemma (1.18), $f$ has a twist orbit of period $n$ for each $n$ such that $3 \mid n$.

Lemma 10.8. Let $k>0$. Let $A_{1}, A_{2}, \ldots, A_{k-1}$ be black arrows and let $A_{k}$ be a colored arrow such that $b\left(A_{i+1}\right) \leq e\left(A_{i}\right)$ for $i=1,2, \ldots, k-1$ and $b\left(A_{1}\right) \leq e\left(A_{k}\right)$. Then $f$ has a periodic orbit $Q$ of period $k$ which is single of the same color as $A_{k}$.

Remark 10.9. Under the assumptions of Lemmas 10.7 and 10.8 and with the notation of Lemma 10.8 , if $A_{k}$ is green then $k \equiv 1$ and if $A_{k}$ is red then $k \equiv 2$.

Proof of Lemma 10.8. Clearly, $k>3$. Set $I_{i}=\left[0, b\left(A_{i}\right)\right]$ for $i=1,2, \ldots, k-$ 1 and $I_{k}=\left[b\left(A_{k-3}\right), b\left(A_{k}\right)\right]$. If $b\left(A_{k-3}\right)<b\left(A_{k}\right)$, then we have $I_{1} \rightarrow I_{2} \rightarrow$ $\cdots \rightarrow I_{k-1} \rightarrow I_{k} \rightarrow I_{1}$. If $b\left(A_{k-3}\right)>b\left(A_{k}\right)$, then (note that by the General Rule $(6.1), k>4)$ we have $I_{1} \rightarrow I_{2} \rightarrow \cdots \rightarrow I_{k-4} \rightarrow I_{k} \rightarrow I_{1}$. In both cases we get a periodic orbit $R=\left\{x_{1}, x_{2}, \ldots, x_{l}\right\}$ of some period $l \leq k$, such that $x_{i} \sim b\left(A_{i}\right)$ for $i=1,2, \ldots, l, f\left(x_{i}\right)=x_{i+1}$ for $i=1,2, \ldots, l-1$, and $f\left(x_{l}\right)=x_{1}$.

We may assume that $R$ is the orbit with the smallest period having these properties (otherwise we take an orbit with the smallest period). We claim that $R$ is single of the same color as $A_{k}$. Otherwise we would have $x_{i}>x_{i+3}$ for some $i \in\{1,2, \ldots, l-3\}$.

If $x_{l-3}>x_{l}$, then

$$
\left[0, x_{1}\right] \rightarrow \cdots \rightarrow\left[0, x_{l-4}\right] \rightarrow\left[x_{l}, x_{l-3}\right] \rightarrow\left[0, x_{1}\right] .
$$

If $x_{l-3}<x_{l}$, then $x_{i}>x_{i+3}$ for some $i<l-3$. Thus,

$$
\left[0, x_{1}\right] \rightarrow \cdots \rightarrow\left[0, x_{i-1}\right] \rightarrow\left[0, x_{i+3}\right] \rightarrow \cdots \rightarrow\left[0, x_{l-1}\right] \rightarrow\left[x_{l-3}, x_{l}\right] \rightarrow\left[0, x_{1}\right] .
$$

In both cases we obtain a periodic orbit with the same properties as $R$ but of a smaller period, which is a contradiction. This proves our claim.

Now if $l=k$, the proof is finished. If $l<k$, then the lemma follows from Lemma 10.7(a) and the Adjusting Lemma (1.18)

Lemma 10.10. If $A$ is a colored arrow, then $f$ has a periodic orbit $Q$ of period smaller than or equal to $m$ which is single of the same color as $A$.

Proof. We consider an integer $k$ and points $x_{0}, x_{1}, \ldots, x_{k-1}$ defined as follows. $x_{0}=\operatorname{Sm}_{\operatorname{ind}(e(A))}$. If $i>0$, then $x_{i}$ is the largest point of $V_{i}$ on the next branch after the one on which $x_{i-1}$ lies. Let $k$ be the smallest integer such that $x_{k-1} \geq b(A)$ (obviously such $k$ exists). Observe that each $x_{i}$ $(i=1,2, \ldots, k-1)$ is an end of an arrow $A_{i}$ beginning in $V_{i-1}$. By Corollary 10.6 , these arrows are black. Since $V_{0} \cap P$ has only one point on each branch, we have $x_{0}=b\left(A_{1}\right)$. Therefore $e(A) \geq b\left(A_{1}\right)$. 
For $i=2,3, \ldots, k-1$ we have $b\left(A_{i}\right) \in V_{i-1}$ and $b\left(A_{i}\right) \sim e\left(A_{i-1}\right)$. The point $e\left(A_{i-1}\right)$ is the largest point of $V_{i-1}$ on its branch. Hence, $e\left(A_{i-1}\right) \geq$ $b\left(A_{i}\right)$. Also, $e\left(A_{k-1}\right) \geq b(A)$. Then, by Lemma 10.8 (set $A_{k}=A$ ), we obtain a periodic orbit $Q$ of $f$ of period $k$ which is single of the same color as $A$.

Since $V_{i}=\operatorname{Span}(P)$ for all $i \geq r$, we have $k-1 \leq r+2$ (we have to add 2 since we have to reach with $x_{i}$ the branch $\left.\operatorname{br}_{\operatorname{ind}(b(A))}\right)$. Therefore, $k \leq m$.

Corollary 10.11. (a) If $P$ has a green arrow and $m \equiv 1$ then $P$ is single green. (b) If $P$ has a red arrow and $n \equiv 2$ then $P$ is single red.

Proof. This follows immediately from Lemma 10.10, Lemma 10.7(a), the Adjusting Lemma (1.18), the First Theorem (2.3), and the assumption that $P$ is primary.

Proof of $(\mathrm{b})$ of Directed Theorem (10.1). Assume that $P$ has both a green and a red arrow. If $m \equiv 0$ then from Lemma 10.10, Lemma 10.7(b), and the Adjusting Lemma (1.18) it follows that $f$ has a twist orbit of period $m$. By the First Theorem (2.3), this is a contradiction. If $m \equiv 1$ or $m \equiv 2$, then it follows from Corollary 10.11 that $P$ is single colored, also a contradiction.

Proof of (c) of Directed Theorem (10.1). If $P$ has only one green (red) arrow, then clearly $m \equiv 1$ (resp. $m \equiv 2$ ). Hence, by Corollary $10.11, P$ is single green (resp. red).

Now, we are left with two cases: either $P$ has only green arrows (at least two of them) and $m \equiv 2$, or $P$ has only red arrows (at least two of them) and $m \equiv 1$. In the rest of this section we shall assume that one of these cases holds and we shall call them green case and red case, respectively.

There is also some difference between the cases of $m$ even and $m$ odd. We shall call these cases even case and odd case, respectively. Thus, we can have four cases: even green, even red, odd green, and odd red.

Our next goal is to prove Directed Theorem $(10.1)(d)$. For this we shall need several lemmas and new notation.

Set $E_{0}=P \cap V_{0}, E_{i}=P \cap\left(V_{i} \backslash V_{i-1}\right)$ for $i=1,2, \ldots, r$. Then $\left\{E_{0}, E_{1}\right.$, $\left.\ldots, E_{r}\right\}$ is a partition of $P$.

For an arrow $A$, denote by $s(A)$ the integer such that $b(A) \in E_{s(A)}$ and by $t(A)$ the integer such that $e(A) \in E_{t(A)}$. Notice that $t(A) \leq s(A)+1$, and if $A$ is a colored arrow, then, by Corollary 10.6, $t(A) \leq s(A)$.

For a colored arrow $A$, set

$$
S(A)=\left\{e(A), f(e(A)), \ldots, f^{(A)}(e(A))\right\},
$$

where

$$
e(A), \ldots, f^{(A)-1}(e(A)) \in V_{s(A)-1}, f^{\prime(A)}(e(A)) \in E_{s(A)} .
$$

Lemma 10.12. If $A, B$ are colored arrows and $A \neq B$, then either

$S(A) \cap S(B)=\varnothing$ or

$S(A) \subset S(B) \backslash\left\{e(B), f^{l(B)}(e(B))\right\}$, or 
$S(B) \subset S(A) \backslash\left\{e(A), f^{l(A)}(e(A))\right\}$.

Proof. Assume that $S(A) \cap S(B) \neq \varnothing$. Then either $e(A) \in S(B)$ or $e(B) \in$ $S(A)$. We may assume that $e(A) \in S(B)$ (in the other case one has to replace $A$ by $B$ and $B$ by $A)$. Since $A \neq B$, we have $e(A) \neq e(B)$. Hence, $b(A) \in$ $S(B) \backslash\left\{f^{l(B)}(e(B))\right\} \subset V_{s(B)-1}$. Thus, $s(A) \leq s(B)-1$. Therefore, by the definition, we obtain $S(A) \subset S(B) \backslash\left\{e(B), f^{l(B)}(e(B))\right\}$.

For a colored arrow $A$, set $T(A)=S(A) \backslash \bigcup\{S(B): B$ is a colored arrow, $B \neq A$, and $S(B) \subset S(A)\}$. By Lemma 10.12, if $A \neq B$, then $T(A) \cap T(B)=$ $\varnothing$. Set $u(A)=s(A)-t(A)+1$.

Lemma 10.13. For every colored arrow $A, \operatorname{Card}(T(A)) \geq u(A)$. If $\operatorname{Card}(T(A))$ $=u(A)$, then no element of $T(A) \backslash\{e(A)\}$ belongs to $E_{0}$.

Proof. We look at the sequence $w_{0}, w_{1}, \ldots, w_{l(A)}$ defined by $f^{i}(e(A)) \in E_{w_{i}}$, $i=0,1, \ldots, l(A)$. This sequence has the following property:

$(* *) \quad$ For each term $v$ of the sequence, the next term is smaller than or equal to $v+1$.

To obtain $T(A)$ from $S(A)$, we have to remove some sets of the form $S(B)$ from it. For the sequence of $w_{i}$ 's this is removing some sequences of the form $w_{j}, w_{j+1}, \ldots, w_{j+l(B)}$ from it. Since then $f^{j-1}(e(A))=b(B)$, we have $w_{j-1}=s(B)$. But also $w_{j+l(B)}=s(B)$. Hence, such an operation again gives a sequence satisfying $(* *)$.

Therefore, the sequence of $w_{i}$ 's corresponding to $T(A)$ also satisfies (**). Hence, its length is greater than or equal to the last term, minus the first term plus 1. By Lemma 10.12, $w_{0}$ and $w_{l(A)}$ are not removed from the sequence. Thus, $\operatorname{Card}(T(A)) \geq w_{l(A)}-w_{0}+1=s(A)-t(A)+1=u(A)$.

If some element of $T(A) \backslash\{e(A)\}$ belongs to $E_{0}$, then the corresponding term $v$ of the sequence of $w_{i}$ 's is $Q$ and the preceding term cannot be equal to $v-1$. Therefore, in this case we obtain a strict inequality.

Lemma 10.14. Let $P$ be a single colored orbit of period $m$ of an EP-adjusted map $f \in \mathbf{Y}$. Then $f$ has periodic orbits of periods $2 m+3,2 m+6,2 m+9, \ldots$ with span strictly smaller than $\operatorname{Span}(P)$.

Proof. We use the notation of the proof of Lemma 10.7. We have the loops

$$
I_{0} \rightarrow I_{1} \rightarrow \cdots \rightarrow I_{m-1} \rightarrow I_{0} \text { and } I_{0} \rightarrow I_{2} \rightarrow I_{0} .
$$

By going around the first loop twice and around the second loop $i$ times $(i \geq 1)$, we obtain a loop of length $2 m+3 i$ which is nonrepetitive. By Lemma 1.3, there is a periodic orbit associated to it. Clearly this periodic orbit has period $2 m+3 i$ and span strictly smaller than $\operatorname{Span}(P)$.

Lemma 10.15. If $A$ is a colored arrow, then $u(A)$ is larger than or equal to $(m-6) / 2$ (resp. $(m-3) / 2)$ in the even (resp. odd) case. If additionally $e(A)$ is the largest point of $V_{t(A)}$ on its branch, then the inequality is strict. 
Proof. We make a construction similar to the one used in the proof of Lemma 10.10. In the general case, $x_{0}$ will be the largest point of $V_{t(A)-1}$ on the same branch as $e(A)$. Also, $x_{i}$ (for $i>0$ ) is the largest point of $V_{t(A)-1+i}$ on the next branch after the one on which $x_{i-1}$ lies. In the special case when $e(A)$ is the largest point of $V_{t(A)}$ on its branch, we set $x_{0}=e(A)$ and $x_{i}$ (for $i>0$ ) is the largest point of $V_{t(A)+i}$ on the next branch after the one on which $x_{i-1}$ lies. Notice that if $t(A)=0$, then necessarily we have this special case. We stop at $x_{k-1}$ when we have for the first time $x_{k-1} \geq b(A)$. Since $b(A) \in V_{s(A)}$, we obtain $t(A)-1+(k-1) \leq s(A)+2(t(A)+(k-1) \leq s(A)+2$ in the special case). As in the proof of Lemma 10.10, at the right-hand side we have to add 2 to $s(A)$ since we have to reach with $x_{i}$ the branch on which $b(A)$ lies.

Now, as in the proof of Lemma 10.10, we use Lemma 10.8 (with $A_{k}=A$ ) and we get a periodic orbit $Q$ of period $k$ which is single of the same color as $A$.

Also, we have $u(A) \geq k-3$, and in the special case the inequality is strict.

Hence, from Lemma 10.14 and the Adjusting Lemma (1.18) it follows that $f$ has periodic orbits of periods $2 k+3 i$ for any integer $i \geq 1$, and all these orbits have span strictly smaller than $\operatorname{Span}(Q)$. Thus, they have span strictly smaller than $\operatorname{Span}(P)$. Since $P$ is primary, from the First Theorem (2.3) it follows that $m \neq 2 k+3 i$ for any integer $i \geq 1$.

We know that in the green case $m \equiv 2$ and $k \equiv 1$, and in the red case $m \equiv 1$ and $k \equiv 2$. Hence, $m \equiv 2 k$ always. Therefore, $m \leq 2 k$.

In the even case we obtain $u(A) \geq k-3 \geq(m-6) / 2$ and in the special case the inequality is strict.

In the odd case, since $m \leq 2 k, m \equiv 2 k$, and $m$ is odd, we get $m \leq 2 k-3$. Hence, we obtain $u(A) \geq k-3 \geq(m-3) / 2$ and in the special case the inequality is strict.

Proof of (d) of Directed Theorem (10.1). Let $v$ be the number of colored arrows. Then $P$ has $m-v$ black arrows. Now we can proceed as in Observation 5.9. In the green case $m-v \equiv 0$, and since $m \equiv 2$ we obtain $v \equiv 2$. In the red case $(m-v)-v \equiv 0$, and since $m \equiv 1$ we also obtain $v \equiv 2$. Hence, in both cases $v \equiv 2$.

The sets $T(A)$ and $T(B)$ for different colored arrows $A$ and $B$ are disjoint. Therefore, by Lemma 10.13 , we obtain $\sum u(A) \leq m$, where the sum is taken over all colored arrows. In view of Lemma 10.15 , we get

$(* * *) \quad m \geq \begin{cases}v_{\cdot}(m-6) / 2 & \text { in the even case, } \\ v_{\cdot}(m-3) / 2 & \text { in the odd case. }\end{cases}$

Assume that the equality holds. Then by Lemmas 10.13 and 10.15 we have:

(i) For each colored arrow $A$, the point $e(A)$ is not the largest point of $V_{t(A)}$ on its branch.

(ii) For each colored arrow $A, \operatorname{Card}(T(A))=u(A)$.

(iii) $\bigcup(T(A))=P$, where the union is taken over all colored arrows. 
Now we look at $\operatorname{sm}_{i}(i \in\{0,1,2\})$. By (iii), it belongs to $T(A)$ for some colored arrow $A$. By (i), it is not equal to $e(A)$. By Lemma 10.13, this contradicts (ii). Hence, the inequality $(* * *)$ is strict.

Since there are at leasi three black arrows and $v \equiv 2$, we have $m \geq 5$. Consider the odd case. We obtain $v<2 m /(m-3)=2+6 /(m-3) \leq 2+$ $6 /(5-3)=5$. Since $v \equiv 2$, we get $v=2$.

Now consider the even case. Since $m \not \equiv 0$, we have $m \geq 8$. If $m \geq 10$, then $v<2 m /(m-6)=2+12 /(m-6) \leq 2+12 /(10-6)=5$. But $v \equiv 2$, and hence $v=2$. Therefore, the only possible exception is $m=8$ and $v=5$. This is in the green case.

We shall examine this exceptional case. $P$ has three black arrows and five green ones. Then by the General Rule (6.1), for each black arrow $A, b(A)$ is the smallest point of $P$ on its branch and $e(A)$ is the largest point of $P$ on its branch. There must be two green arrows, $B$ and $C$, such that $e(B)=b(C)$ and $e(C)$ is the smallest point of $P$ on its branch. We may assume that this branch is $b_{0}$. We consider the intervals $I=[b(B), e(B)], J=[b(C), e(C)]$, $M=[0, e(C)], K=\left[0, \mathrm{sm}_{1}\right], L=\left[0, \mathrm{sm}_{2}\right]$. Then we have

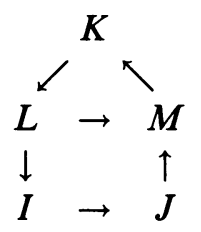

and we obtain the loop $K \rightarrow L \rightarrow M \rightarrow K \rightarrow L \rightarrow I \rightarrow J \rightarrow M \rightarrow K$ of length 8. Clearly this loop gives us a periodic orbit of period 8. This orbit is different from $P$ because it has only two green arrows (given by $I \rightarrow J$ and $J \rightarrow M$ ). From the First Theorem (2.3) this is a contradiction with the primarity of $P$. Thus, the case $m=8$ and $v=5$ is not possible. This ends the proof of (d) of the Directed Theorem.

It remains to prove statements (e) and (f) of the Directed Theorem (10.1) In the even case set $r=(m-2) / 2$.

Lemma 10.16. Let $m$ be even. Let $A$ and $B$ be colored arrows, $A \neq B$. Then there exists an integer $p$ such that $0 \leq p \leq r$ and $f^{p}(e(A))=b(B)$.

Proof. Clearly, there exists an integer $p$ such that $0 \leq p \leq m-2$ and $f^{p}(e(A))$ $=b(B)$. Suppose that $p>r$. Set $x=f^{p-r}(e(A))$. Then $f^{r}(x)=b(B)$.

In the green case $r \equiv 0$ and in the red case $r \equiv 1$. By (d) of the Directed Theorem (10.1), all arrows along the orbit from $x$ to $b(B)$ are black. Hence, in both cases $x \sim e(B)$.

Set $q=m-2-p$. We then have $f^{q}(e(B))=b(A)$. Since $p>r$ and $p+q=2 r$, then $q+1 \leq r$. Now, we consider two possibilities (notice that $x=e(B)$ is impossible).

1. $x<e(B)$. We set $K=[0, b(B)], L=[0, x], M=[x, e(B)]$. Clearly $K$ $f$-covers $L$ and $M$. Since $f^{\prime}(x)=b(B)$ and $f^{\prime}(0)=0, L f$-covers $K$ in 
$r$ steps. Moreover, since the arrows along the orbit from $x$ to $b(B)$ are black, going from $L$ to $K$, we always move from one branch to the next one. Since $q+1 \leq r, f^{q+1}(x)$ and $f^{q+1}(e(B))=e(A)$ lie on different branches. Hence, $0 \in f^{q+1}(M)$ and consequently $0 \in f^{r}(M)$. Also, $b(B)=f^{r}(x) \in f^{r}(M)$. Thus, $M f$-covers $K$ in $r$ steps. Here we also follow the arrows along the orbit from $x$ to $b(B)$ and therefore we always move from one branch to the next one. We obtain the loop

$$
K \rightarrow M \underset{r \text { steps }}{\longrightarrow} K \rightarrow L \underset{r \text { steps }}{\longrightarrow} K,
$$

where we do not move to the next branch but to the same one (green case) or to the previous one (red case) only when we go from $K$ to $M$ and from $K$ to $L$. Since $L \cap M=\{x\} \in P$, this loop gives us a periodic orbit of period $m$. Since $P$ is primary, from the First Theorem (2.3) this orbit is equal to $P$. But for this orbit $p=r$, a contradiction.

2. $x>e(B)$. We set $K=[0, e(A)], L=[0, e(B)], M=[e(B), x]$, and we have $f^{p-r}(0)=0, f^{p-r}(e(A))=x$. Hence, $K f$-covers $L$ and $M$ in $p-r$ steps. Since we follow the orbit of $x$ for $p-r<p$ steps, we always move from one branch to the next one. Since $f^{q+1}(0)=0$ and $f^{q+1}(e(B))=e(A)$, $L f$-covers $K$ in $q+1$ steps. Here we move from one branch to the next one except at the last step, when we follow $A$. Since $q+1 \leq r$, the points $f^{q+1}(x)$ and $f^{q+1}(e(B))=e(A)$ lie on different branches. Hence, $M f$-covers $K$ in $q+1$ steps. Here again we move from one branch to the next one except at the last step, when we follow $A$. Then we have the loop

$$
K \underset{p-r \text { steps }}{\longrightarrow} M \underset{q+1 \text { steps }}{\longrightarrow} \underset{p-r \text { steps }}{\longrightarrow} \underset{q+1 \text { steps }}{\longrightarrow} K \text {. }
$$

We do not move to the next branch but to the same one (green case) or to the previous one (red case) only when we make the last step from $L$ to $K$ and the last step from $M$ to $K$. Since $L \cap M=\{e(B)\} \subset P$, this loop gives us a periodic orbit of period $2(p-r+q+1)=m$. Since $P$ is primary, by the First Theorem, this orbit is equal to $P$. But for this orbit $p=r$, a contradiction.

Corollary 10.17. In the even case, if $A$ and $B$ are colored arrows and $A \neq B$, then $f^{r}(e(A))=b(B)$ and $f^{r}(e(B))=b(A)$.

Proof. From Lemma 10.16, we have $f^{p}(e(A))=b(B)$ for some $p$ such that $0 \leq p \leq r$. If we apply Lemma 10.16, with $A$ instead of $B$ and vice versa, we obtain $f^{q}(e(B))=b(A)$ for some $q$ such that $0 \leq q \leq r$. Clearly $p+q=$ $m-2=2 r$. Hence $p=q=r$.

In the rest of this section we take $A, p, x, i$ and $B, q, y, j$ as in Definition 4.27. Also, we set $n=(m-5) / 2$ if $m$ is odd (as in Definition 4.27) and $n=r-3=(m-8) / 2$ if $m$ is even. Since $m \geq 5, m \equiv 2$ in the green case, and $m \equiv 1$ in the red case, we have $n \geq 0$. Also we note that $n \equiv 0$ in the green case and $n \equiv 1$ in the red case. 
Lemma 10.18. $y_{j} \sim x_{p-n+j}$ for $\max (0, n-p) \leq j \leq \min (n, q)$.

Proof. First consider the case $p \geq n$. Since $p-n \leq p$ we have $y_{0}=$ $f^{n+1}\left(x_{p-n}\right), \operatorname{ind}\left(y_{0}\right)-\operatorname{ind}\left(x_{p-n}\right) \equiv n$ in the green case, and ind $\left(y_{0}\right)-\operatorname{ind}\left(x_{p-n}\right)$ $\equiv n-1$ in the red case. In both cases ind $\left(y_{0}\right)-\operatorname{ind}\left(x_{p-n}\right) \equiv 0$ and $y_{0} \sim x_{p-n}$. Since $\min (n, q) \leq p$ and $\min (n, q) \leq q$, this ends the proof in the case $p \geq n$.

Now assume that $p<n$. By Corollary $10.17, m$ is odd. Then $q+p=2 n+3$ and $q>n-p$. Hence, $y_{n-p}=f^{p+1+n-p}\left(x_{0}\right)=f^{n+1}\left(x_{0}\right)$, ind $\left(y_{n-p}\right)-\operatorname{ind}\left(x_{0}\right) \equiv$ $n$ in the green case, and ind $\left(y_{n-p}\right)-\operatorname{ind}\left(x_{0}\right) \equiv n-1$ in the red case. As above we get $y_{n-p} \sim x_{0}$. Since $p<n$ and $p+q=2 n+3$ we have $q>n+3$. Hence, $p-n+\min (n, q)=p$. Since $\min (n, q) \leq q$, this ends the proof in the case $p<n$.

Lemma 10.19. In the even case, if conditions (iii.1), (iii.2), (iii.3), and (iii.4) of Definition 4.27 are satisfied, then $P$ is a box orbit of the same color as $A$ and $B$.

Proof. From Corollary 10.17. we have $p=q=n+3$. Hence, $p-n=$ $q-n=3, \max (0, n-p)=\max (0, n-q)=0$, and $\min (n, p)=\min (n, q)=$ $n$. From (iii.1) and (iii.3) it follows that $x_{0} \sim y_{0}$. Hence, $y_{i} \sim x_{i}$ for $i=0,1,2, \ldots, n+3$. Also, from (iii.1), (iii.2), (iii.3), and (iii.4) it follows that $\max \left\{x_{i}, y_{i}\right\}<\min \left\{x_{i+3}, y_{i+3}\right\}$ for $i=0,1,2, \ldots, n$. Clearly, $P$ is a 2-extension of a periodic orbit $Q$ of period $n+4$, and $Q$ is a single orbit of the same color as $A$ and $B$. By Definition 4.23, $P$ is a box orbit.

Proof of (e) and (f) of Directed Theorem (10.1). From Lemma 10.19 and Definition 4.27 it follows that it is enough to show that conditions (iii.1), (iii.2), (iii.3), and (iii.4) of Definition 4.27 are satisfied.

From Lemma 10.18 we have $y_{j} \sim x_{p-n+j}$ for all $j$ such that $\max (0, n-p) \leq$ $j \leq \min (n, q)$. Suppose that for some $j, y_{j}>x_{p-n+j}$. Consider the arrows $A_{1}=\left(y_{0}, y_{1}\right), \ldots, A_{j}=\left(y_{j-1}, y_{j}\right), A_{j+1}=\left(x_{p-n+j}, x_{p-n+j+1}\right), \ldots, A_{n}=$ $\left(x_{p-1}, x_{p}\right), A_{n+1}=B=\left(x_{p}, y_{0}\right)$. By Lemma 10.8, $f$ has a periodic orbit $Q$ of period $n+1$ which is single of the same color as $B$. From Lemma 10.14 and the Adjusting Lemma (1.18), $f$ has periodic orbits of periods $2(n+1)+3$ and $2(n+1)+6$ with span strictly smaller than $\operatorname{Span}(Q)$. Thus, they have span strictly smaller than $\operatorname{Span}(P)$. If $m$ is odd, then $2(n+1)+3=m$, and if $m$ is even, then $2(n+1)+6=m$. From the First Theorem (2.3) this is a contradiction. This proves that (iii.1) is satisfied.

By replacing $A, p, x, i$ by $B, q, y, j$ and vice versa, we obtain (iii.2).

To prove (iii.3) and (iii.4) we shall consider separately the odd and even cases.

Even case. From Corollary 10.17 it follows that $p=q=n+3$. Hence, $y_{0}=$ $f^{n+4}\left(x_{0}\right)$. Then, similarly to the proof of Lemma 10.18 we get $y_{0} \sim x_{0}$. We may assume that $y_{n+3}=b(A)<b(B)=x_{n+3}$. Assume that $x_{i}>x_{i+3}$ for some $i \in\{0,1,2, \ldots, n\}$. Consider arrows $A_{1}=\left(x_{0}, x_{1}\right), \ldots, A_{i}=\left(x_{i-1}, x_{i}\right)$, $A_{i+1}=\left(x_{i+3}, x_{i+4}\right), \ldots, A_{n}=\left(x_{n+2}, x_{n+3}\right), A_{n+1}=A=\left(b(A), x_{0}\right)$. Since 
$x_{n+3}>b(A)$, we can use Lemma 10.8 and we obtain a periodic orbit $Q$ of period $n+1$, which is single of the same color as $A$. In the same way as before, this is a contradiction. This proves (iii.3).

Suppose now that $y_{j}>y_{j+3}$ for some $j \in\{0,1, \ldots, n\}$. We cannot just replace $A, p, x, i$ by $B, q, y, j$ and vice versa, because we have the assumption that $b(A)<b(B)$. Hence, we have to use more complicated arguments. Notice that $\left[0, x_{n+2}\right] f$-covers $\left[x_{n+3}, y_{n+3}\right]$ because $y_{n+3}<x_{n+3} .\left[x_{n+3}, y_{n+3}\right] f$ covers $\left[x_{0}, y_{0}\right] .\left[x_{j-1}, y_{j-1}\right] f$-covers $\left[x_{j}, y_{j+3}\right]$ because $x_{j}<y_{j+3}<y_{j}$ (here we use (iii.2)). $\left[x_{n}, y_{n+3}\right] f$-covers $\left[0, x_{0}\right]$ because $y_{n+3}=b(A), e(A)=x_{0}$, and $\left(x_{n}, x_{n+1}\right)$ is a black arrow. Also, $\left[0, x_{2}\right] f$-covers $\left[0, x_{0}\right]$ because $x_{3}>$ $x_{0}$. Therefore we have the loop

$$
\begin{aligned}
& {\left[0, x_{0}\right] \rightarrow\left[0, x_{1}\right] \rightarrow\left[0, x_{2}\right] \rightarrow\left[0, x_{0}\right] \rightarrow \cdots \rightarrow\left[0, x_{n+2}\right] \rightarrow\left[x_{n+3}, y_{n+3}\right]} \\
& \quad \rightarrow\left[x_{0}, y_{0}\right] \rightarrow \cdots \rightarrow\left[x_{j-1}, y_{j-1}\right] \rightarrow\left[x_{j}, y_{j+3}\right] \rightarrow \cdots \rightarrow\left[x_{n}, y_{n+3}\right] \rightarrow\left[0, x_{0}\right]
\end{aligned}
$$

of length $3+(n+3)+1+n+1=2 n+8=m$. When we move along it, we move to the next branch each time except the steps $\left[x_{n+3}, y_{n+3}\right] \rightarrow\left[x_{0}, y_{0}\right]$ and $\left[x_{n}, y_{n+3}\right] \rightarrow\left[0, x_{0}\right]$. Since the numbers of steps between these two moves, from the first to the second and from the second to the first, are not equal (they are $n+6$ and $n$, respectively), we obtain a periodic orbit of period $m$ from the loop. From the First Theorem, this orbit is $P$. But for this orbit we have $p \neq q$, a contradiction. This proves (iii.4).

Odd case. We may assume that $p>q$. Since $p+q=2 n+3$ we have $q-3<$ $n<p$. Then (iii.4) holds from Lemma 4.32(b).

To prove (iii.3), by Lemma $4.32(\mathrm{a})$, it is enough to prove that $x_{i}<x_{i+3}$ only for $0 \leq i<n-q$ (if $n-q>0$ ) and for $\min (n, p-3)<i \leq p-3$ (if $n<p-3)$.

Suppose that $x_{i}<x_{i+3}$. Consider the case $0 \leq i<n-q$. Then $q<n$ and, by (iii.1), we obtain $y_{q}<x_{p-n+q}=x_{n+3}$. We take the arrows $A_{1}=$ $\left(x_{0}, x_{1}\right), \ldots, A_{i}=\left(x_{i-1}, x_{i}\right), A_{i+1}=\left(x_{i+3}, x_{i+4}\right), \ldots, A_{n}=\left(x_{n+2}, x_{n+3}\right), A_{n+1}$ $=A=\left(y_{q}, x_{0}\right)$. Since $x_{i}>x_{i+3}$, by Lemma 10.8, we obtain a contradiction in the same way as before.

Consider the case $\min (n, p-3)<i \leq p-3$. Then $p-3>n$, which implies $q<n$. By (iii.2) we have $x_{n-q}<y_{0}$. We take the arrows $A_{1}=$ $\left(x_{n-q}, x_{n-q+1}\right), \ldots, A_{i-n+q}=\left(x_{i-1}, x_{i}\right), A_{i+1-n+q}=\left(x_{i+3}, x_{i+4}\right), \ldots, A_{n}=$ $\left(x_{p-1}, x_{p}\right), A_{n+1}=B=\left(x_{p}, y_{0}\right)$ (we have $i>n-q$ since $i>\min (p-3, n)=$ $n)$. Since $x_{i}>x_{i+3}$, by Lemma 10.8 we obtain a contradiction in the same way as before. This ends the proof of (iii.3).

\section{DEPENDENCES BETWEEN PERIODIC ORBITS}

In this section we study which primary orbits are forced and not forced by other primary orbits (see Remark 9.2). In this and the next sections, we must sometimes again consider $\{0\}$ as a primary orbit of $f \in \mathbf{Y}$ of period 1. It will be clear where we do that. 
Lemma 11.1. If $P$ is a pendulum orbit of an EP-adjusted map $f \in \mathbf{X}$, then $f$ has no directed orbits.

Proof. In cases I, II, and III (here we use the notation of $\S 4$ ) it is obvious. So we assume that we have case IV. If there is no arrow beginning and ending on two different even branches, it is also obvious. Hence, we assume that $P$ has an arrow $A$ such that $b(A)$ and $e(A)$ lie on different even branches. Since we have case IV and $P$ is pendulum we know that the period $m$ of $P$ is odd, $m \geq 5$, and $A$ is the only blue arrow of $P$.

Assume that $Q$ is a directed periodic orbit of $f$. Since $A$ is the only blue arrow of $P$, all arrows of $Q$ beginning and ending on even branches, have to begin in the $E P$-basic interval $J$ adjacent to $b(A)$ and end in the interval $(0, e(A))$. Since $Q$ is directed, the smallest point of $Q$ on the same branch as $b(A)$ has to belong to $J$ and the arrow $B$ beginning at it ends in $(0, e(A))$. Since $b(A)$ is the largest point of $P$ on its branch, all points of $Q$ on this branch belong to $J$. Since $f$ is $E P$-adjusted, $f(e(B)) \in(0, f(e(A)))$. By the definition of pendulum orbit (Definition 4.1), $f(e(A))$ is the smallest point of $P$ on the odd branch. Let $x$ be the smallest point of $Q$ on the odd branch. Then $x \in(0, f(e(B))] \subset(0, f(e(A)))$ and $f(x) \sim b(B)$. Since $f$ is $E P$-adjusted, it follows that $f^{2}(e(A)) \sim b(B) \sim b(A)$. Since $m>3, f^{2}(e(A))<b(A)$. Therefore, $\left(0, f^{2}(e(A))\right) \cap J=\varnothing$. Thus, $f(x)<f^{2}(e(A))<b(B)$. This is a contradiction because $b(B)$ is the smallest point of $Q$ on its branch.

Lemma 11.2. Let $P$ be a periodic orbit of an EP-adjusted map $f \in \mathbf{Y}$. Assume that $P$ is primary and directed. Then $f$ has no undirected orbits.

Proof. Assume that $f$ has an undirected orbit $Q$. There are two possibilities.

1. There is an arrow $A$ of $Q$ such that $b(A)<e(A)$. Consider the $E P$ basic interval $[x, y]$ such that $x<b(A)<y$. Since $f$ is $E P$-adjusted, either $f(x)$ or $f(y)$ is larger than $e(A)$. By the General Rule (6.1) this cannot be $f(x)$. Hence $f(y)>e(A)$. By the General Rule we have $y>f(y)$ and, consequently, $y>f(y)>e(A)>b(A)>x$, contrary to the assumption that $[x, y]$ was $E P$-basic.

2. There is no such arrow of $Q$. Then $Q$ is not contained in one branch. Since $Q$ is undirected, there are two arrows of $Q, A$ and $B, A \neq B$, such that $b(A)$ and $b(B)$ are the smallest points of $Q$ on their branches, $e(A) \geq b(B)$ and $e(B) \geq b(A)$. Consider the $E P$-basic intervals $\left[x_{A}, y_{A}\right],\left[x_{B}, y_{B}\right]$ such that $x_{A}<b(A)<y_{A}$ and $x_{B}<b(B)<y_{B}$. Since they are $E P$-basic intervals and $f$ is $E P$-adjusted, one of the points $x_{A}, y_{A}$ is mapped to a point larger than or equal to $y_{B}$, and one of the points $x_{B}, y_{B}$ is mapped to a point larger than or equal to $y_{A}$. This contradicts the Directed Rule (10.4).

Lemma 11.3. With the assumptions of Lemma 11.2, if $Q$ is a periodic orbit of $f$, then

(a) If $P$ has no green arrows, then $Q$ has no green arrows.

(b) If $P$ has no red arrows, then $Q$ has no red arrows. 
Proof. By Lemma 11.2, $Q$ is directed. Therefore, talking about green and red arrows of $Q$ is justified.

(a) Assume that $P$ has no green arrows. Since $f$ is $E P$-adjusted, there is no point $x \in Y \backslash\{0\}$ such that $x \sim f(x)$. Consequently, $Q$ has no green arrows.

(b) Assume that $P$ has no red arrows. Since $f$ is $E P$-adjusted, there is no point $x \in Y \backslash\{0\}$ such that $x$ is on the next branch after $f(x)$. Consequently, the direction of black arrows of $Q$ is the same as the direction of black arrows of $P$, and $Q$ has no red arrows.

Lemma 11.4. Let $P$ be a box green (red) orbit of period $m$ of an EP-adjusted map $f \in \mathbf{Y}$. Then $f$ has a single green (resp. red) orbit of period $m / 2$.

Proof. By the definition of box colored orbit (Definition 4.2) $P$ is a 2-extension of a single colored orbit $Q$ of the same color as $P$. Let $P_{i}(i=0,1, \ldots, m / 2$ -1 ) be the sets from the definition of $n$-extension (Definition 3.1). Then $f^{m / 2}$ has a fixed point in $\operatorname{Span}\left(P_{0}\right)$. Clearly, its $f$-orbit is equivalent to $Q$.

Lemma 11.5. Let $P$ be a double colored orbit of period $m$ of an EP-adjusted map $f \in \mathbf{Y}$. Then $f$ has a periodic orbit of period $m+3$.

Proof. From Lemmas 5.15 and 5.16 it follows that the loop of the EP-graph of $f$ associated to $P$ goes through all basic intervals. Adding to this loop the loop of length $3\left[0, \mathrm{sm}_{0}\right] \rightarrow\left[0, \mathrm{sm}_{1}\right] \rightarrow\left[0, \mathrm{sm}_{2}\right] \rightarrow\left[0, \mathrm{sm}_{0}\right]$, we obtain a loop of length $m+3$. This loop is nonrepetitive, and hence the periodic orbit associated to it has period $m+3$.

Lemma 11.6. Let $P$ be a periodic orbit of period $m$ of an EP-adjusted map $f \in \mathbf{Y}$. Then

(a) If $P$ is single green (red), then $f$ has a double green (resp. red) orbit of period $2 m+3$.

(b) If $P$ is double green (red), then $f$ has a box green (resp. red) orbit of period $m+3$.

Proof. (a) By Lemma 10.14, $f$ has a periodic orbit of period $2 m+3$. By the Primary Theorem (2.5), $f$ has a primary periodic orbit $Q$ of period $2 m+3$. By Lemma 11.2, $Q$ is directed. By Lemma 11.3, $Q$ has no red (resp. green) arrows. Since $m \equiv 1$ (resp. $m \equiv 2$ ) we have $2 m+3 \equiv 2$ (resp. $2 m+3 \equiv 1$ ). Hence, by (d) of the Directed Theorem (10.1), $Q$ has exactly two green (resp. red) arrows. Therefore, by (f) of the Directed Theorem, $Q$ is double green (resp. red).

(b) By Lemma 11.5, $f$ has a periodic orbit of period $m+3$. As above, by the Primary Theorem and Lemmas 11.2 and 11.3, $f$ has a primary orbit $Q$ of period $m+3$ which is directed and has no red (resp. green) arrows. Since $m \equiv 2$ (resp. $m \equiv 1$ ) we have $m+3 \equiv 2$ (resp. $m+3 \equiv 1$ ). By (d) and (e) of the Directed Theorem, we have that $Q$ has two green (resp. red) arrows and, hence, $Q$ is box green (resp. red). 
Lemma 11.7. Let $P$ be a pendulum orbit of period $m$ of an EP-adjusted map $f \in \mathbf{X}$. Assume that $m=2^{k} . n, k>0, n>1, n$ odd. Then $f$ has orbits of all periods $s$ such that $s>_{s} m$.

Proof. By the definition of pendulum orbits (Definition 4.1), $P$ is an $R$-extension of a pendulum orbit $Q$ of period $2^{k}$, for some pendulum orbit $R$ of period $n$. We may assume that the maps $g$ and $h$ for which $R$ and $Q$ are periodic orbits, respectively, are $R$-adjusted and $E Q$-adjusted, respectively. By Lemma 7.2 and Remark 5.1, $g$ has periodic orbits of all periods $l$, where $l$ is either odd and larger than $n$ or even. By Lemma 3.10, $f$ has periodic orbits of periods $2^{k} l$ for $l$ as above. By Lemma 5.2 and Remark 5.1, $h$ has periodic orbits of periods $2^{i}, i=0,1, \ldots, k-1$, and $Q$ itself has period $2^{k}$. By Lemma 3.11, $f$ has periodic orbits of periods $2^{i}, i=0,1, \ldots, k$. Now the statement of the lemma follows from the definition of Šarkovskii ordering.

Proposition 11.8. Let $P$ be a pendulum orbit of period $m$ of an EP-adjusted map $f \in \mathbf{X}$. If $s>_{s} m$, then $f$ has a pendulum orbit of period $s$.

Proof. From Lemmas 7.2, 5.2, 11.7 and Remark 5.1, it follows that $f$ has a periodic orbit of period $s$. By the Primary Theorem (2.5), $f$ has a primary periodic orbit of period $s$. By Lemma 11.1, this orbit is undirected. From the Undirected Theorem (7.1) it is pendulum.

Proposition 11.9. Let $P$ be a twist orbit of period 3.l of an EP-adjusted map $f \in \mathbf{Y}$. If $s>_{s} l$, then $f$ has a twist orbit of period $3 s$.

Proof. If $l=1$, then there is nothing to prove. If $l=2^{k}$, from Lemma 5.5 it follows that $f$ has a periodic orbit $Q$ of period $3 s$. If $l=2^{k} . n, n$ odd, and $n \geq 3$, then $P$ is an $R$-extension of a twist orbit of period $3.2^{k}$, and $R$ is a pendulum orbit of period $n$. We may assume that the map $g$ for which $R$ is a periodic orbit is $R$-adjusted. By Proposition 11.8, $g$ has a periodic orbit $R^{\prime}$ of period $s$. Since $s \neq n, R^{\prime} \neq R$. By Lemma 3.10, $f$ has a periodic orbit $Q \neq P$ of period $3.2^{k} s$.

From the Primary Theorem (2.5) in both cases, we may assume that $Q$ is primary. By Lemmas 11.2 and 11.3, $Q$ is directed and has no colored arrow. From (a) of the Directed Theorem (10.1), $Q$ is twist.

From now on, a periodic orbit will be called green if and only if it is either single green or double green or box green. Similarly, it will be called red if and only if it is either single red or double red or box red.

The following theorem states that some primary orbits force some other ones.

Theorem 11.10 (Forcing Theorem). Let $f \in \mathbf{Y}$ and let $P$ be a primary periodic orbit of $f$ of period $m>1$. Then the following hold.

(a) If $P$ is pendulum, then $f$ has a pendulum orbit of period $n$ for each $n$ such that $n>{ }_{s} m$.

(b) If $P$ is twist, then $f$ has a twist orbit of period $n$ for each $n$ such that $n \equiv 0$ and $n / 3>_{s} m / 3$. 
(c) If $P$ is green, then $f$ has a green orbit of period $n$ for each $n$ such that $n \not \equiv 0, n>_{g} m$, and $n>1$, and a twist orbit of period $n$ for each $n$ such that $n \equiv 0$.

(d) If $P$ is red, then $f$ has a red orbit of period $n$ for each $n$ such that $n \not \equiv 0, n>r, m$, and $n>1$, and a twist orbit of period $n$ for each $n$ such that $n \equiv 0$.

Proof. (a) follows from Proposition 11.8 and the Adjusting Lemma (1.18). (b) follows from Proposition 11.9 and the Adjusting Lemma. If $f$ is $E P$-adjusted, from Lemmas 11.6 and 11.4 we have that $f$ has a green (resp. red) orbit of period immediately following $m$ in the green (resp. red) ordering. The Adjusting Lemma allows us to skip the assumption that $f$ is $E P$-adjusted. In such a way we obtain the first part of (c) and (d) by induction. The second part of (c) and (d) follows from Lemma 10.7(b) and the Adjusting Lemma.

Theorem 11.11 (Periods Theorem). Let $f \in \mathbf{Y}$. Then

(a) The set of periods of all pendulum orbits of $f$ is of the form $S\left(n_{s}\right)$ for some $n_{s} \in \mathbf{N}_{s}$.

(b) The set of periods of all green and twist orbits of $f$ is of the form $G\left(n_{g}\right) \backslash\{1\}$ for some $n_{g} \in \mathbf{N}_{g}$.

(c) The set of periods of all red and twist orbits of $f$ is of the form $R\left(n_{r}\right) \backslash\{1\}$ for some $n_{r} \in \mathbf{N}_{r}$.

(d) $\operatorname{Per}(f)=S\left(n_{s}\right) \cup G\left(n_{g}\right) \cup R\left(n_{r}\right)$.

(e) $n_{s}, n_{g}$, and $n_{r}$ appearing in (a), (b), and (c) are determined uniquely by $f$.

Proof. (a), (b), and (c) follow immediately from the Forcing Theorem (11.10) and the definitions of orderings. (e) is an obvious consequence of (a), (b), and (c).

Now we prove (d). By the Primary Theorem (2.5), $\operatorname{Per}(f)$ is equal to the set of periods of all primary periodic orbits of $f$. By the Undirected Theorem (7.1), the Directed Theorem (10.1), and (a), (b), and (c) we obtain (d).

Notice that $n_{s}, n_{g}$, and $n_{r}$ which appear in (a) of the Main Theorem (see Introduction), are not determined uniquely by $f$. Hence, the Periods Theorem is a stronger (and more precise) version of (a) of the Main Theorem.

The following theorem states that some primary orbits do not force some other ones.

Theorem 11.12 (Nonforcing Theorem). Let $P$ be a periodic orbit of period $m>$ 1 of an EP-adjusted map $f \in \mathbf{Y}$. Also let $Q \neq P$ be a primary periodic orbit of $f$ of period $n>1$. Then the following hold.

(a) If $P$ is pendulum, then $Q$ is pendulum and $n>_{s} m$.

(b) If $P$ is twist, then $Q$ is twist and $n / 3>_{s} m / 3$.

(c) If $P$ is green, then $Q$ is either green or twist and $n>_{g} m$.

(d) If $P$ is red, then $Q$ is either red or twist and $n>_{r} m$. 
Proof. In all cases, by Propositions 5.4, 5.7, 5.17, and 5.18, $P$ is primary. By the First Theorem (2.3), $P$ is the only periodic orbit of $f$ of period $m$. Hence, $n \neq m$. Also in all cases, since $f$ is $E P$-adjusted, we have $\operatorname{Span}(Q) \subsetneq \operatorname{Span}(P)$.

(a) By Lemma 11.1 and the Undirected Theorem (7.1), $Q$ is pendulum. If $m>_{s} n$, then by the Forcing Theorem (11.10) and the Adjusting Lemma (1.18), $f$ has a periodic orbit $R$ of period $m$ with $\operatorname{Span}(R) \subsetneq \operatorname{Span}(Q)$. Hence $R \neq P$, a contradiction.

(b) (resp. (c), (d)). By Lemmas 11.2 and 11.3 and the Directed Theorem (10.1), $Q$ is twist (resp, green or twist, red or twist). If $m / 3>_{s} n / 3$ (resp. $\left.m>_{g} n, m>_{r} n\right)$ then we get a contradiction as in the proof of $(\mathbf{a})$.

Theorem 11.13 (Adjusted Periods Theorem). (a) Let $P$ be a periodic orbit of period $m>1$ of an EP-adjusted map $f \in \mathbf{Y}$. Then

(a.1) If $P$ is pendulum, then $\operatorname{Per}(f)=S(m)$.

(a.2) If $P$ is green or twist, then $\operatorname{Per}(f)=G(m)$.

(a.3) If $P$ is red or twist, then $\operatorname{Per}(f)=R(m)$.

(b) There exist maps $f$ and $g \in \mathbf{Y}$ such that each periodic orbit of $f$ (resp. $g$ ) of period larger than one is pendulum (resp. twist) and $\operatorname{Per}(f)=S\left(2^{\infty}\right)$ (resp. $\operatorname{Per}(g)=G\left(3.2^{\infty}\right)=R\left(3.2^{\infty}\right)$ ).

Proof. (a) By the Primary Theorem (2.5), $\operatorname{Per}(f)$ is equal to the set of periods of all primary orbits of $f$. Hence, (a) follows from the Forcing Theorem (11.10) and the Nonforcing Theorem (11.12).

(b) It is known (see, e.g., [Ša]) that there exists a map $h \in \mathbf{I}$ such that $\operatorname{Per}(h)=S\left(2^{\infty}\right)$ and, for each $i \geq 1, h$ has only one periodic orbit of period $2^{i}$. Moreover, $h(0)=0$ and $h$ has only one fixed point different from 0 . Since $[0,1] \subset Y$, we can define $f$ by $f_{\mid[0,1]}=h, f(x)=0$ for $x \notin[0,1]$. Clearly, $f \in \mathbf{Y}, \operatorname{Per}(f)=S\left(2^{\infty}\right)$, and for each $i \geq 1, f$ has only one periodic orbit of period $2^{i}$ (we recall that in the case of maps of $Y$, we do not consider $\{0\}$ as a periodic orbit). Hence, all these periodic orbits are primary. By construction, they are undirected. Then, by the Undirected Theorem (7.1), they are pendulum.

Let $\varepsilon=\exp (2 \pi i / 3)$. We define $g$ by $g(x)=\varepsilon . h(x)$ for $x \in[0,1]$ and $g(x)=\varepsilon x$ for $x \notin[0,1]$. Clearly, $g \in \mathbf{Y}, \operatorname{Per}(g)=G\left(3.2^{\infty}\right)=R\left(3.2^{\infty}\right)$, and for each $i \geq 0, g$ has only one periodic orbit of period $3.2^{i}$. Hence, all these orbits are primary. By construction they are directed and have only black arrows. Then, by the Directed Theorem (10.1), they are twist.

\section{COEXISTENCE OF PERIODIC ORBITS}

In this section we are going to get the tool to prove (b) of the Main Theorem.

Theorem 12.1 (Coexistence Theorem). Let $P_{i}$ be a primary periodic orbit of a map $f_{i} \in \mathbf{Y}, i=1,2, \ldots, n$. Then there exists a map $f \in \mathbf{Y}$ such that:

(a) $f$ has periodic orbits $Q_{i}$ E-equivalent to $P_{i}$ for $i=1,2, \ldots, n$. 
(b) For each periodic orbit $R$ of $f$, there is $i \in\{1,2, \ldots, n\}$ such that $f_{i}$ has a periodic orbit of the same period as $R$.

Proof. We use induction on $n$. For $n=1$, the statement is obviously true. Assume that it is true for $n-1$ and prove it for $n$.

There exists a map $g \in \mathbf{Y}$ such that it has a periodic orbit $Q E$-equivalent to $P_{n}$ and $g$ is $E Q$-adjusted. Set $Z=\bigcup_{i=0}^{\infty} g^{-i}(0) \backslash\{0\}$. There exists a map $\psi: Y \rightarrow Y$, mapping each branch onto itself in a nondecreasing way and such that $\psi^{-1}(x)$ consists of only one point if and only if $x \notin Z \cup\{0\}$ and $\psi^{-1}(0)$ is homeomorphic to $Y$. In other words, $\psi^{-1}$ blows up 0 and all its inverse images under iterates of $g$. If $x \in Z$, then $\psi^{-1}(x)$ is an interval.

We define $f$ in several steps. If $\psi(x) \notin Z \cup\{0\}$, then $g \circ \psi(x) \notin Z \cup\{0\}$ and we set $f(x)=\psi^{-1}(g \circ \psi(x))$. If $y \in Z$ then, since $g$ is $E Q$-adjusted, there exists one-sided limits (from outside) of $f(z)$ as $z$ tends to the endpoints of $\psi^{-1}(y)$. Moreover, these limits are equal to the endpoints of the interval $\psi^{-1}(g(y))$ if $g(y) \in Z$ or to extremal points of $\psi^{-1}(0)$ if $g(y)=0$. Then we extend $f$ to the interval $\psi^{-1}(y)$ continuously to its endpoints and linearly to the whole interval. If $x$ is an extremal point of $\psi^{-1}(0)$ then, since $g$ is $E Q$ adjusted, there exists a one-sided limit (from outside) of $f(z)$ as $z$ tends to $x$, and it is equal to an extremal point of $\psi^{-1}(0)$. We extend $f$ continuously to $x$.

In such a way $f$ is defined and continuous on the set $\overline{Y \backslash \psi^{-1}(0)}$. There exists a homeomorphism $\varphi$ of $Y$ onto a subset $\tilde{Y}$ of $\operatorname{Int}\left(\psi^{-1}(0)\right)$. By the induction hypothesis, there exists a map $\tilde{f} \in \mathbf{Y}$ such that $\tilde{f}$ has periodic orbits $\tilde{Q}_{i}, i=1,2, \ldots, n-1, E$-equivalent to $P_{i}$, and if $R$ is a periodic orbit of $\tilde{f}$ then there is $i \in\{1,2, \ldots, n-1\}$ such that $f_{i}$ has a periodic orbit of the same period as $R$. We set $f=\varphi \circ \tilde{f} \circ \varphi^{-1}$ on $\tilde{Y}$. On the remaining three intervals joining $\overline{Y \backslash \psi^{-1}(0)}$ with $\tilde{Y}$, we extend $f$ linearly.

In such a way $f$ is defined on the whole $Y$. Clearly $f$ satisfies (a). We shall show that $f$ also satisfies (b).

Let $R$ be a periodic orbit of $f$. If $R \cap \tilde{Y} \neq \varnothing$ then, since $\tilde{Y}$ is $f$ invariant, $R \subset \tilde{Y}$. Then by the definition of $\left.f\right|_{\tilde{Y}},(\mathrm{~b})$ is satisfied.

Assume that $R \cap \tilde{Y}=\varnothing$ but $R \cap \psi^{-1}(0) \neq \varnothing$. Since $\psi^{-1}(0)$ is $f$-invariant, $R \subset \psi^{-1}(0) \backslash \tilde{Y}$. This set consists of three intervals $I_{0}, I_{1}, I_{2}\left(I_{i} \subset \mathrm{br}_{i}\right)$. By the definition of $f$, for each $i$ there exists $j$ such that $f\left(I_{i}\right) \subset I_{j} \cup \tilde{Y}$. Since $g$ is $E Q$-adjusted, $j=\operatorname{ind}\left(g\left(\mathrm{sm}_{i}\right)\right)$. Since $\left.f\right|_{I_{i}}$ is linear for $i=0,1,2$ and $\tilde{Y}$ and the set of extremal points of $\psi^{-1}(0)$ are $f$-invariant, either $R$ is contained in the set of the extremal points of $\psi^{-1}(0)$ or for each $x \in R$, if $x \in I_{i}$, then the whole $I_{i}$ consists of points of periodic orbits $E$-equivalent to $R$. In both cases, there is a periodic orbit $R^{\prime}$ contained in the set of extremal points of $\psi^{-1}(0)$ and $E$-equivalent to $R$. If $Q$ is directed, then $R^{\prime}$ is a twist periodic orbit of period 3. Since $Q$ is primary, by the Directed Theorem (10.1) 
and the Forcing Theorem (11.10), $g$ also has such orbit. If $Q$ is undirected then $R^{\prime}$ has period 1 or 2 . However, by the Undirected Theorem (7.1) and the Forcing Theorem, $g$ also has such orbit.

Now assume that $R \cap \psi^{-1}(0)=\varnothing$. Then $R \cap \psi^{-1}(Z \cup\{0\})=\varnothing$, and $\varphi(R)$ is a periodic orbit of $g, E$-equivalent to $R$.

Hence, in all cases when $R \cap \tilde{Y}=\varnothing$ we get a periodic orbit of $g$ of the same period as $R$. By the Adjusting Lemma (1.17), $f_{n}$ also has such an orbit. This proves (b).

Remark 12.2. One can prove a stronger version of the above theorem. Let $P_{i}$ be a periodic orbit of a map $f_{i} \in \mathbf{Y}, i=1,2, \ldots, n$. Then there exists $f \in \mathbf{Y}$ such that

(a) $f$ has periodic orbits $Q_{i}, i=1,2, \ldots, n, E$-equivalent to $P_{i}$.

(b) For each periodic orbit $R$ of $f$ there is $i \in\{1,2, \ldots, n\}$ such that $R$ is $E$-equivalent to some periodic orbit of $f_{i}$.

This version has no assumption that $P_{i}$ is primary and has a stronger condition (b). It can also be generalized for trees $\left\{z \in \mathbf{C}: z^{s} \in[0,1]\right\}$. The proof, instead of using the Forcing Theorem (11.10), uses the following lemma: If $P$ is a periodic orbit of $g, g(0)=0, i_{1}, \ldots, i_{n}$ are pairwise different numbers such that $\operatorname{ind}\left(g\left(\operatorname{sm}_{i j}\right)\right)=i_{j+1}$ for $j=1,2, \ldots, n-1$, and $\operatorname{Ind}\left(g\left(\operatorname{sm}_{i_{n}}\right)\right)=i_{1}$, then there exists an orbit $\left\{x_{1}, x_{2}, \ldots, x_{n}\right\}$ of $g$ such that $g\left(x_{j}\right)=x_{j+1}$ for $j=1,2, \ldots, n-1, g\left(x_{n}\right)=x_{1}$, and $\operatorname{ind}\left(x_{j}\right)=i_{j}$ for $j=1,2, \ldots, n$. However, we do not need this stronger version of the Coexistence Theorem, and we leave its proof (and the proof of the lemma!) to the interested readers.

\section{PROOF OF MAIN THEOREM, FINAL REMARKS, AND CONJECTURES}

Proof of Main Theorem. (a) follows from the Periods Theorem (11.11). (b) follows from the Adjusted Periods Theorem (11.18), the Coexistence Theorem (12.1), and Remarks 4.17, 4.22, 4.26, 4.39, and 4.43.

Remark 13.1. Instead of using the above remarks, we can use only the existence of pendulum orbits of period 3 , double green orbits of period 5 , double red orbits of period 7, and the Forcing Theorem (11.10).

As an easy corollary to the Main Theorem, we obtain the following result of Mumbru [Mu].

Theorem 13.2. (a) If $f \in \mathbf{Y}$ and $\{2,3,4,5,7\} \subset \operatorname{Per}(f)$, then $\operatorname{Per}(f)=\mathbf{N}$.

(b) If $W \subset \mathbf{N}$ is a set such that for every $f \in \mathbf{Y}, W \subset \operatorname{Per}(f)$ implies $\operatorname{Per}(f)=\mathbf{N}$, then $W \subset\{2,3,4,5,7\}$.

Remark 13.3. Since the results on pendulum orbits are valid for $I$ as well as for $\mathbf{Y}$, in the same way as in the proof of the Main Theorem, we can obtain an alternative proof of the Sarkovskii Theorem.

Conjecture 13.4. Let $k>3$, let $Z=\left\{z \in \mathbf{C}: z^{k} \in[0,1]\right\}$, and let $\mathbf{Z}$ be the set of all continuous maps of $Z$ into itself for which 0 is a fixed point. Then 
there exist total (linear) orderings $<_{1}, \ldots,<_{l}$ of subsets $W_{1}, \ldots, W$ of $\mathbf{N}$, respectively, such that:

(a) If $f \in \mathbf{Z}$, then $\operatorname{Per}(f)=W_{1}^{f} \cup \cdots \cup W_{l}^{f}$ where the sets $W_{i}^{f} \subset W_{i}$ have the following property: if $m>_{i} n$ and $n \in W_{i}^{f}$, then $m \in W_{i}^{f}$.

(b) If the sets $W_{i}^{\prime} \subset W_{i}(i=1,2, \ldots, l)$ have the property that if $m>_{i} n$ and $n \in W_{i}^{\prime}$, then $m \in W_{i}^{\prime}$, then there exists $f \in \mathbf{Z}$ such that $\operatorname{Per}(f)=$ $W_{1}^{\prime} \cup W_{2}^{\prime} \cup \cdots \cup W_{l}^{\prime}$.

In Conjecture 13.4 we may replace $\mathrm{Z}$ by other sets of maps. For example,

(1) The set of all continuous maps of a given tree into itself for which all branching points are fixed points.

(2) The set of all continuous maps of $Y$ into itself.

(3) The set of all continuous maps of $Z$ into itself.

(4) The set of all continuous maps of a given tree into itself.

In such a way we obtain new conjectures (or perhaps rather open problems).

Remark 13.5. If in Conjecture 13.4 we replace $\mathbf{Z}$ by the set of all continuous maps of a circle into itself of degree one, then it becomes false (consider rotations).

\section{REFERENCES}

[AL1] L. Alsedà and J. Llibre, A note on the set of periods for continuous maps of the circle which have degree one, Proc. Amer. Math. Soc. 93 (1985), 133-138.

[AL2] __ Orbites périodiques minimales des applications continues du cercle dans lui même ayant un point fixe, C. R. Acad. Sci. Paris 301 (1985), 601-604.

[ALMS] L. Alsedà, J. Llibre, M. Misiurewicz, and C. Simó, Twist periodic orbits and topological entropy for continuous maps of the circle of degree one which have a fixed point, Ergodic Theory Dynamical Systems 5 (1985), 501-517.

[ALS] L. Alsedà, J. Llibre, and R. Serra, Minimal periodic orbits for continuous maps of the interval, Trans. Amer. Math. Soc. 286 (1984), 595-627.

[Ba] S. Baldwin, Generalizations of a theorem of Šarkovskii on orbits of continuous real-valued functions, Discrete Math. 67 (1987), 111-122.

[Be1] C. Bernhardt, Periodic points of a class of endomorphisms of the circle, J. London Math. Soc. 25 (1982), 539-550.

[Be2] __, Periodic points and topological entropy of maps of the circle, Proc. Amer. Math. Soc. 87 (1983), 516-518.

[Be3] __ Periodic orbits of continuous mappings of the circle without fixed points, Ergodic Theory Dynamical Systems 1 (1981), 413-417.

[Be4] _ , The ordering on permutations induced by continuous maps of the real line, Ergodic Theory Dynamical Systems 7 (1987), 155-160.

[B11] L. Block, Mappings of the interval with finitely many periodic points have zero entropy, Proc. Amer. Math. Soc. 67 (1977), 357-360.

[B12] __ Homoclinic points of mappings of the interval, Proc. Amer. Math. Soc. 72 (1978), 576580.

[B13] __ Simple periodic orbits of mappings of the interval, Trans. Amer. Math. Soc. 254 (1979), 391-398. 
[B14] __ Periodic orbits of continuous mappings of the circle, Trans. Amer. Math. Soc. 260 (1980), 555-562.

[B15] _ Periods of periodic points of maps of the circle which have a fixed point, Proc. Amer. Math. Soc. 82 (1981), 481-486.

[BC] L. Block and W. A. Coppel, Stratification of continuous maps of an interval, Trans. Amer. Math. Soc. 297 (1986), 587-604.

[BCJM] L. Block, E. Coven, L. Jonker, and M. Misiurewicz, Primary orbits of maps of the circle, preprint, 1987.

[BCN] L. Block, E. Coven, and Z. Nitecki, Minimizing topological entropy for maps of the circle, Ergodic Theory Dynamical Systems 1 (1981), 145-149.

[BCMN] L. Block, E. Coven, I. Mulvey, and Z. Nitecki, Homoclinic and nonwandering points for maps of the circle, Ergodic Theory Dynamical Systems 3 (1983), 521-532.

[BGMY] L. Block, J. Guckenheimer, M. Misiurewicz, and L. S. Young, Periodic points and topological entropy of one dimensional maps, Lecture Notes in Math., Vol. 819, Springer, Berlin, 1980, pp. 18-34.

[BH1] L. Block and D. Hart, Statification of the space of unimodal interval maps, Ergodic Theory Dynamical Systems 3 (1983), 533-539.

[BH2] _ Orbit types for maps of the interval, preprint, 1986.

[C] W. A. Coppel, Šarkovskiǐ-minimal orbits, Math. Proc. Cambridge Philos. Soc. 93 (1983), 397408.

[E] L. S. Efremova, Periodic points of a continuous map of a circle (in Russian), Proc. IX Internat. Conf. on Nonlinear Oscillations, Kiev, 1981, p. 121.

[H] C. W. Ho, On the structure of the minimum orbits of periodic points for maps of the real line, preprint, 1984.

[HM] C. W. Ho and C. Morris, A graph theoretic proof of Sharkovsky's Theorem on the periodic points of continuous functions, Pacific J. Math. 96 (1981), 361-370.

[I] W. Imrich, Periodic points of small periods of continuous mappings of trees, Ann. Discrete Math. 27 (1985), 443-446.

[IK] W. Imrich and R. Kalinowski, Periodic points of continuous mappings of trees, Ann. Discrete Math. 27 (1985), 447-460.

[It] R. Ito, Minimal entropy for endomorphisms of the circle, Proc. Amer. Math. Soc. 86 (1982), 321-327.

[JR] L. Jonker and D. Rand, The periodic orbits and entropy of certain maps of the unit interval, J. London Math. Soc. 22 (1980), 175-181.

[LMPY] T. Li, M. Misiurewicz, G. Pianigiani, and J. Yorke, No division implies chaos, Trans. Amer. Math. Soc. 273 (1982), 191-199.

[LY] T. Li and J. Yorke, Period three implies chaos, Amer. Math. Monthly 82 (1975), 985-992.

[LR] J. Llibre and A. Reventós, On the structure of the set of periodic points of a continuous map of the interval with finitely many periodic points, Arch. Math. (Basel) 39 (1982), 331-334.

[Mi] M. Misiurewicz, Periodic points of maps of degree one of a circle, Ergodic Theory Dynamical Systems 2 (1982), 221-227.

[MN] M. Misiurewicz and Z. Nitecki, Combinatorial patterns and entropy for interval maps, preprint 1987.

[Mu] P. Mumbrú, Periodes 1, 2, 3, 4, $5 i 7$ equivalen a caos, Master's Thesis, Barcelona, 1982.

[N] Z. Nitecki, Topological dynamics on the interval, Ergodic Theory and Dynamical Systems, Vol. II (Proc. Special Year, Maryland, 1979-1980), Progress in Math., Birkhäuser, Boston, Mass., 1981.

[Ša] A. N. Šarkovskii, Coexistence of cycles of a continuous map of a line into itself, Ukrain. Mat. Z. 16 (1964), 61-71.

[Si] H. W. Sieberg, Chaotic mappings on $S^{1}$, Report No. 37, University of Bremen, 1980. 
[Št] P. Štefan, A theorem of Šarkovskii on the existence of periodic orbits of continuous endomorphisms of the real line, Comm. Math. Phys. 54 (1977), 237-248.

[Str] P. Straffin, Periodic points of continuous functions, Math. Mag. 51 (1978), 99-105.

Departament de Matemàtiques, Facultat de Ciències, Universitat Autònoma de Barcelona, 08193 Bellaterra, Barcelona, Spain

Departament d'Economía i H. Economica, Facultat de Ciencies Economiques, Universitat Autonoma de Barcelona, 08193 Bellaterra, Barcelona, Spain

Instytut Matematyki, Uniwersytet Warszawski, Pakac Kultury i Nauki IX P., 00-91 Warszawa, Poland 